\title{
ASSESSING ORGANIZATIONAL CAPACITY TO SUPPORT RESEARCH \\ UTILIZATION IN ONTARIO: A DESCRIPTIVE STUDY AMONG SENIOR NURSING \\ LEADERS
}

\author{
by \\ Tanvi Sharma \\ Bachelor of Science in Nursing, \\ Ryerson University, Toronto, Ontario, 2012
}

\author{
A thesis \\ presented to Ryerson University \\ in partial fulfillment of the \\ requirements for the degree of \\ Master of Nursing \\ In the Program of \\ Nursing
}

Toronto, Ontario, Canada, 2015

(C) Tanvi Sharma 2015 


\section{Author's Declaration}

I hereby declare that I am the sole author of this thesis or dissertation.

I authorize Ryerson University to lend this thesis or dissertation to other institutions or individuals for the purpose of scholarly research.

I further authorize Ryerson University to reproduce this thesis or dissertation by photocopying or by other means, in total or in part, at the request of other institutions or individuals for the purpose of scholarly research.

I understand that my thesis may be made electronically available to the public. 


\title{
ASSESSING ORGANIZATIONAL CAPACITY TO SUPPORT RESEARCH UTILIZATION IN ONTARIO: A DESCRIPTIVE STUDY AMONG SENIOR NURSING LEADERS
}

\author{
Master of Nursing, 2015 \\ Tanvi Sharma \\ Master of Nursing Program \\ Ryerson University
}

\begin{abstract}
Purpose: To examine the extent to which senior nursing leaders report the presence of components of organizational capacity that support research use; and to describe the facilitators and barriers to research utilization.

Methods: A survey method involving a sample of 43 senior nursing leaders was used in this descriptive study.

Results: The components that participants reported as most frequently available were organizational culture and values and supporting continuing professional development. The components that were reported as least frequently available were setting priorities for obtaining research and assessing quality and applicability of research. The top three facilitators were: networks, availability of resources and organizational support. The top three barriers were: lack of resources, an unsupportive organization and RN attitudes/beliefs.

Conclusions: The results of this study highlight the need for organizations to consider culture and values, leadership support, education and training and resource allocation in order to increase organizational capacity to support research utilization.
\end{abstract}




\section{Acknowledgments}

Without the support of many individuals, this thesis would not have been possible.

Firstly, thank you to Dr. Suzanne Fredericks for being so patient with me and always offering suggestions to enhance the final product. Secondly, I must thank Dr. Heather Beanlands for introducing me to quantitative research methods, asking those tough (but important) questions and being such a critical mentor throughout this process. Lastly to my thesis supervisor, Dr. Cristina Catallo, thank you for providing your expertise, assistance and guidance, believing in me and igniting an interest to pursue a thesis during my undergraduate education.

Secondly, I express my sincere appreciation and gratitude to the senior nursing leaders who took the time to participate in this thesis. Your dedication to enhancing the nursing profession, coupled with years of experience has made this thesis what it is.

I must also thank my papa, mama and sister Rita for always encouraging me and believing in me. Thank you for understanding when I could not spend as much time as I wanted with the family. Without your support, it would not have been possible to concentrate on or devote time to this thesis. Thanks for your pep talks and prayers for me papa and thank you for constantly caring for me when I was locked in my room for hours at a time mama. I love you guys so much and thank you for helping me get through this! Also a big thank you to countless friends and family members for their continuous words of encouragement.

Lastly, to my husband to be- Nikhil, thank you for believing in me, never letting me give up, and teaching me the importance of patience. Thank you for understanding me, giving me space when I needed it and cheering me along the way! I could not have done this without your love and support. I cannot wait to finally start the next chapter of our life together and be able to make up for so much lost time. I love you and can't thank you enough for always being with me. 


\section{Dedication}

I would like to dedicate this thesis to my papa and mama, without whom I would not have been able to pursue or achieve this dream of mine. Thank you for working long hours and sacrificing your career papa, just so I never had to work during my undergraduate or graduate degree. You always told me to focus on school and it is because of your hard work that I am where I am today. And of course my mama, you took care of me and were there on the days and nights when I felt like giving up. Both of your hugs, words of encouragement and support are what got me through this. I love you so much for helping me in so many ways throughout these three years and no words can come close to expressing how blessed I feel to have you both as my parents. This achievement is just as much yours as it is mine and it was only possible because you chose to provide me the best education that you could 18 years ago. I look forward to finally being able to spend more time with you! 


\section{Table of Contents}

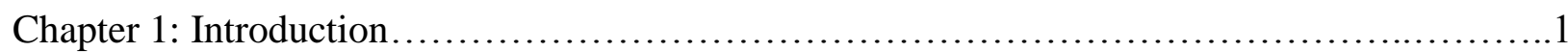

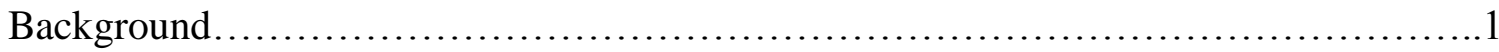

Exploration of Individual Attributes........................................4

Nature of Research........................................................

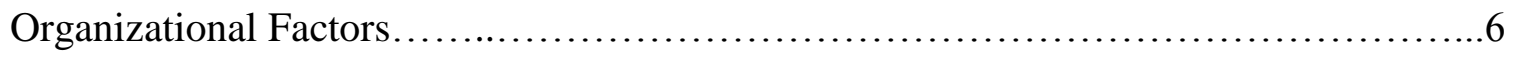

Organizational capacity.............................................

Components of organizational capacity............................8

Organizational Capacity and Senior Nursing Leaders............................. 9

Senior nursing leaders............................................11

Statement of the Problem...................................................... 11

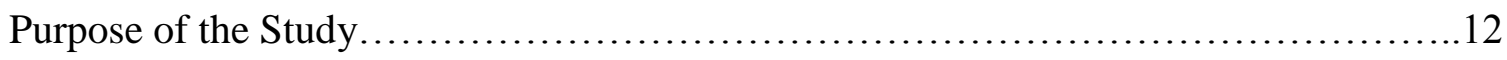

Significance.......................................................... 12

Chapter 2: Literature Review.................................................... 15

Literature Search Strategy...............................................15

Results of the search............................................... 16

Quality of Included Literature............................................17

Qualitative literature.............................................. 17

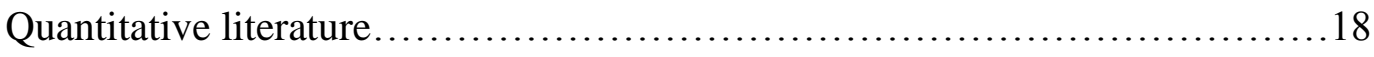

Systematic reviews..............................................20

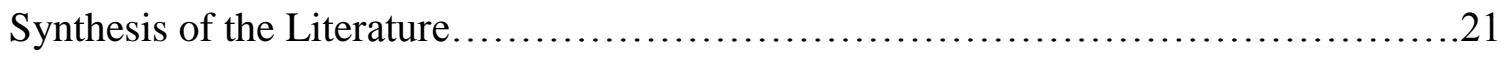

Individual Attributes and their Impact on Research Utilization among

Nurses........................................................21 


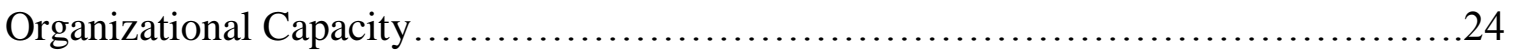

Organizational culture and values and setting priorities to obtain research.......24

Organizational culture ................................................

Mission and vision................................................ 28

Leadership support............................................28

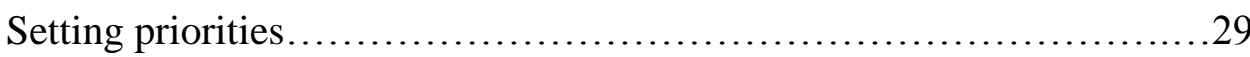

Obtaining, critically appraising and using research in practice.................29

Lack of time....................................................... 30

Lack of resources................................................ 30

Lack of mentors................................................... 31

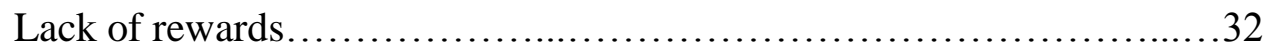

Monitoring and evaluating policies and programmes.........................32

Professional development.................................................

Literature with Senior Nursing Leaders...........................................35

Summary of the Literature Review.............................................. 38

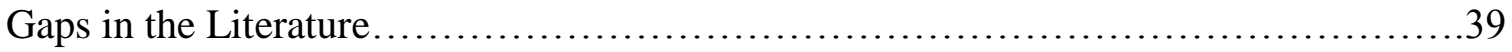

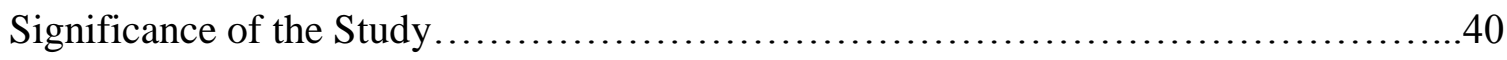

Need for research focused on organizational capacity in relation to research

utilization.............................................................. 40

Need for quantitative research..........................................40

Need for research with senior nursing leaders..............................40

Need for research from Ontario............................................41

Chapter 3: Theoretical Framework....................................................... 43 
Use of the term Research Utilization.............................................43

Use of the SUPPORT Series as a Guiding Perspective..............................44

Evidence-Informed Health Policymaking ..........................................45

Steps of Evidence-Informed Health Policymaking..................................46

Improving how an organization supports EIHP ............................46

Assessing organizational capacity to use research evidence..............47

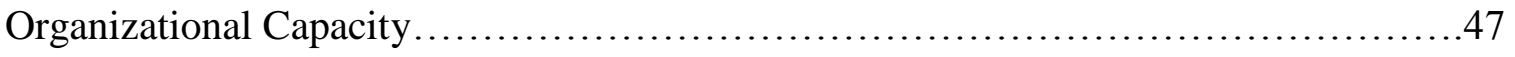

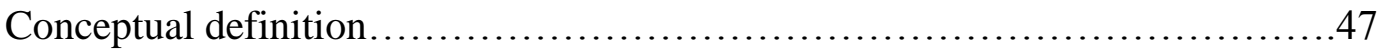

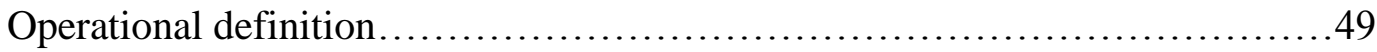

Organizational culture and values..................................50

Setting priorities to obtain research...................................50

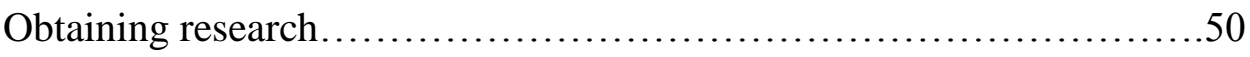

Assessing the quality and applicability of research and interpreting the results.......................................................... 50

Using research................................................ 50

Monitoring and evaluation..........................................51

Supporting continuing professional development.....................51

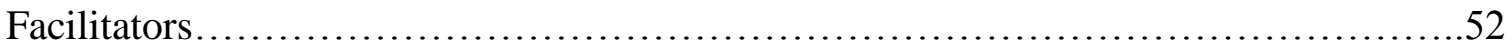

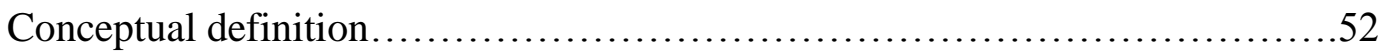

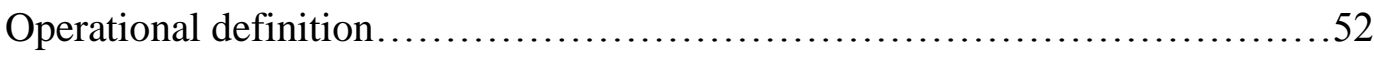

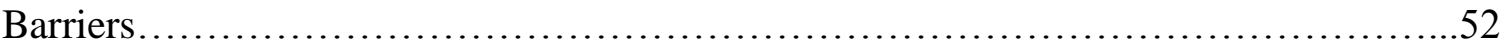

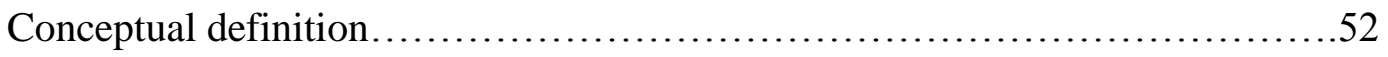

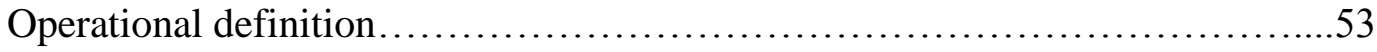


Research Questions.......................................................... 53

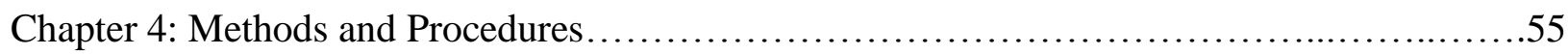

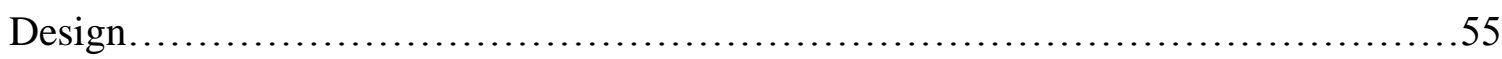

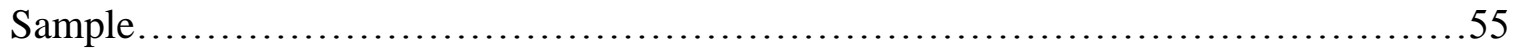

Target and accessible population........................................55

Inclusion and exclusion criteria........................................55

Sampling method and recruitment......................................57

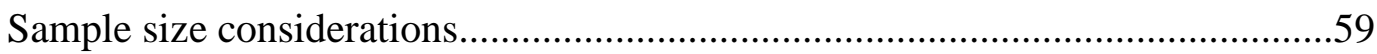

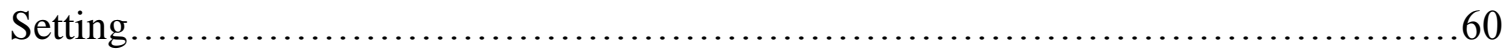

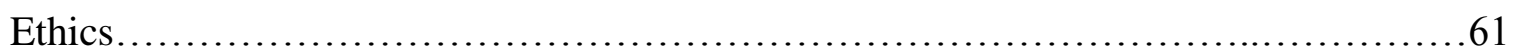

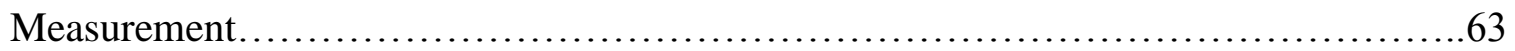

Demographic Data Questionnaire........................................63

Organizational Capacity to Support the Use of Research Evidence to Inform

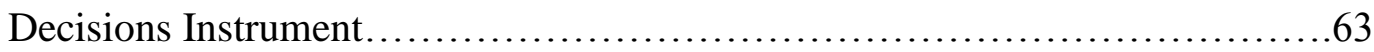

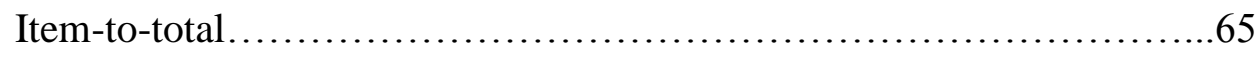

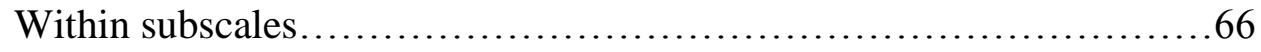

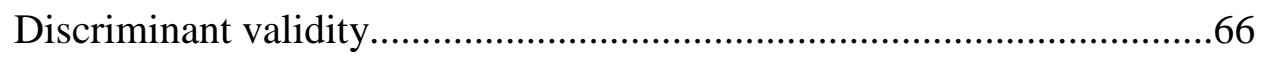

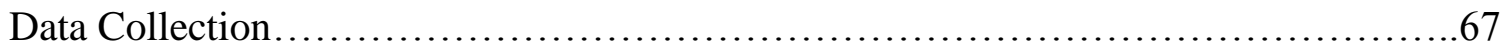

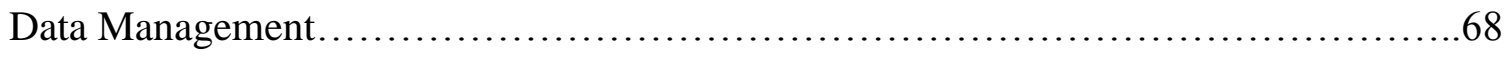

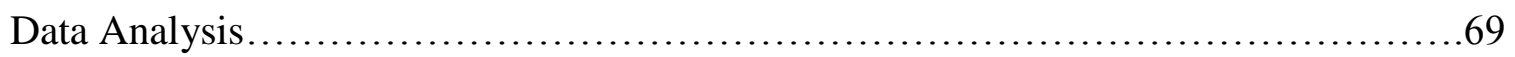

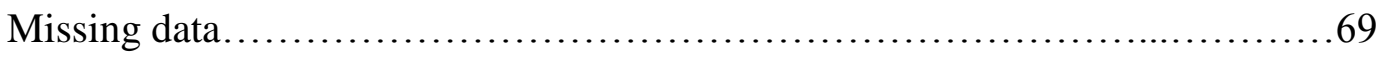

Summary of missing data.......................................... 70

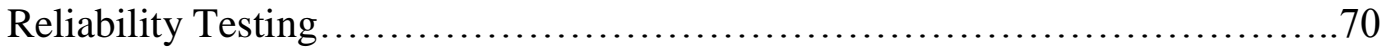


Statistical Analysis.

Sample........................................................

Research questions..........................................71

Chapter 5: Results............................................................. 75

Sample............................................................... 75

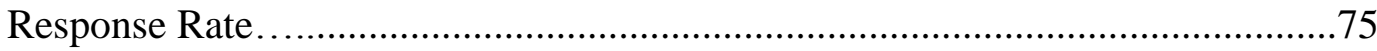

Demographic Profile..................................................76

Organizational Capacity Instrument Reliability Testing...........................78

Findings Related to the Research Questions.....................................79

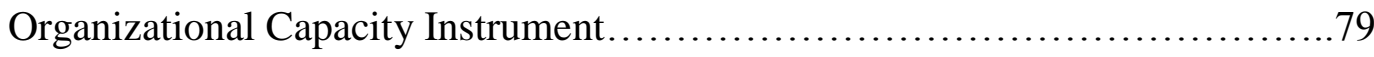

Interpretation of the mean subscale scores.......................................80

Interpretation of the item mean scores...........................8 81

Facilitators and Barriers to Research Utilization............................85

Facilitators of Research Utilization...............................85

Networks...........................................86

Resources................................................86

Organizational support..................................86

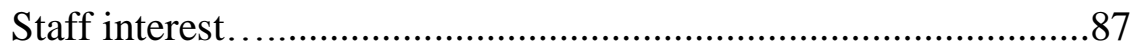

Creating accountability..................................87

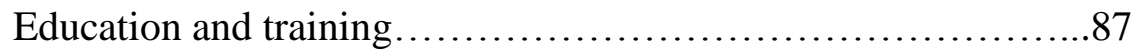

Barriers to Research Utilization........................................................8

Lack of resources............................................................ 88

Unsupportive organization................................ 89 
Nurse attitudes and beliefs..................................................89

Organization size........................................99

Constitution of 'evidence' ..................................90

Other................................................... 90

Chapter 6: Discussion....................................................... 91

Representativeness of the Sample............................................91

Reliability Testing of the Organizational Capacity Instrument.....................92

Discussion of Key Findings............................................... 92

Comparison of mean scores with Catallo and Sidani (2014)..................92

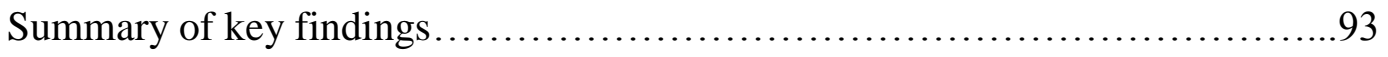

Neutral Findings among Item Mean Scores.......................................................94

Organizational Culture and Values Support the Use of Research....................96

Key organizational documents supporting research utilization...............96

Support from leadership ...........................................97

Setting Priorities for Obtaining Research......................................98

Assessing the Quality and Applicability of Research and Interpreting the Results.....100

Having skilled staff.............................................. 100

Continuing Professional Development......................................... 102

Absence of the Provision of Time, Incentives, Resources, Expertise and Access to

Experts.............................................................. 104

Availability of time and resources..................................... 105

Availability of incentives........................................... 106

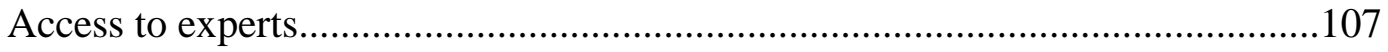


Facilitators and Barriers to Research Utilization................................109

Networks as a facilitator...............................................109

Nurse attitudes and beliefs as a barrier.................................. 110

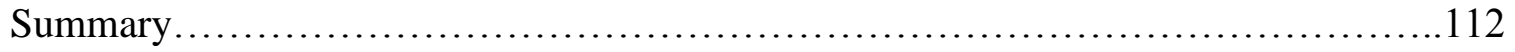

Study Limitations..................................................... 113

Sampling ........................................................113

Recruitment.......................................................... 114

Measurement......................................................115

Validity testing of the Organizational Capacity Instrument............116

Unsystematic threats to internal validity.........................116

Missing data.................................................117

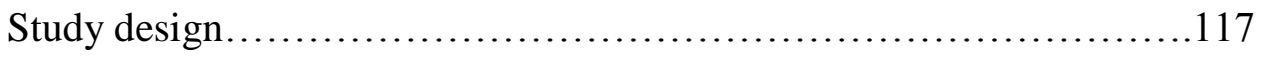

Chapter 7: Summary, Implications and Conclusion.................................119

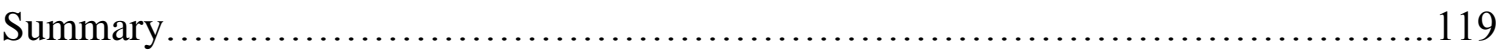

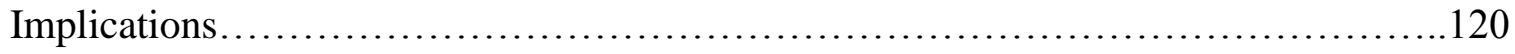

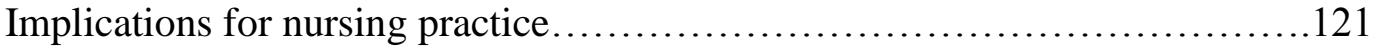

Implications for education....................................... 125

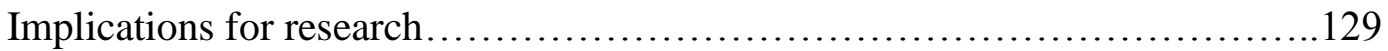

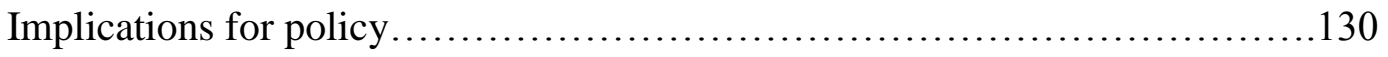

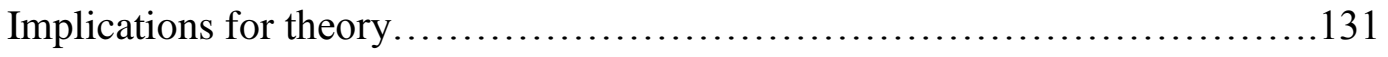

Conclusion..............................................................131

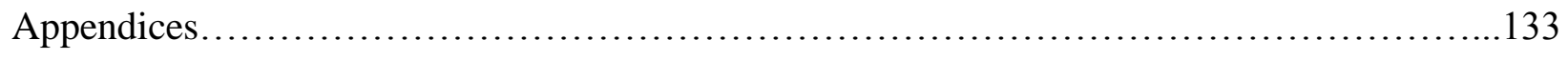

References.................................................................... 142 


\section{List of Tables}

Table 1. Age and number of years as a nurse, nursing leader and in current position............76

Table 2. Other demographic characteristics of the sample.............................. 78

Table 3. Cronbach's Alpha Coefficient for The Organizational Capacity Instrument and

Subscales............................................................. 79

Table 4. Descriptive Statistics for subscales of the Organizational Capacity Instrument

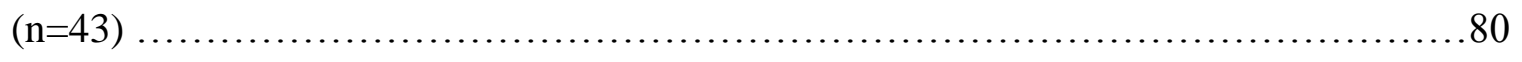

Table 5. Descriptive Statistics for items of the Organizational Capacity Instrument

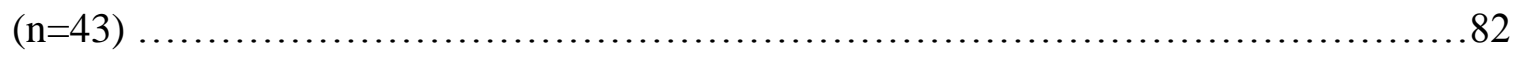

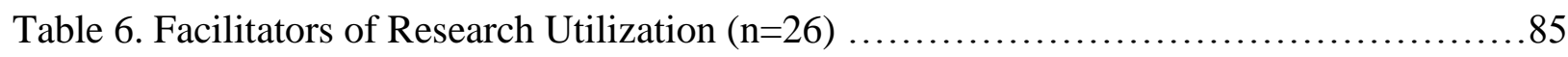

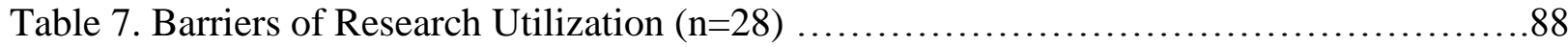




\section{List of Appendices}

Appendix A: First Literature Search Results..........................................133

Appendix B: Second Literature Search Results..................................... 134

Appendix C: Online NLN.ON Recruitment Advertisement............................ 135

Appendix D: Ryerson University Research Ethics Board Approval.........................136

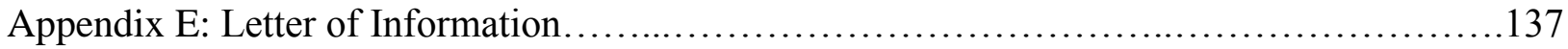

Appendix F: Demographic Data Questionnaire........................................ 140 


\section{Chapter 1: Introduction}

\section{Background}

Research is considered to be one of the most rigorous forms of evidence in healthcare.

When used by healthcare professionals, research can contribute to an enhanced healthcare system that provides high-quality and safe care to patients while improving outcomes based on practices that are substantiated by research (Straus, Tetroe \& Graham, 2009). These benefits are possible as the use of research challenges nurses to think critically about traditional practices and procedures and also question those that have not been substantiated by research. Various terms describing the use of research have been presented in the literature. Evidence-based medicine is perhaps the earliest term, giving rise to evidence-based practice and evidence-based nursing (Jolley, 2013). Other terms such as knowledge translation, knowledge transfer, knowledge exchange, research utilization, implementation and, more recently, evidence-informed decisionmaking have emerged.

Although there are differences among the terms, the common theme among them is the notion of incorporating research within practice. The term research utilization (RU) is used in this thesis and is defined as "the use of research findings in any and all aspects of one's work as a registered nurse" (Estabrooks, 1998, p.19). Although a variety of terms that capture the essence of using research are available, the term research utilization was selected for its broad conceptualization. This study did not aim to focus on specific aspects of practice that could be informed by research, so a broad term that was concerned with use of research was sought. It is also important to note that research in this study, refers to systematic reviews, meta-analyses, integrative reviews, randomized control trials, quasi-experimental studies, correlational studies, qualitative research studies, meta-summaries and meta-synthesis (Burns \& Grove, 2009). 
Knowledge generation among disciplines, particularly in nursing, is growing at an accelerated rate, also creating the need to explore the utilization of this knowledge. Combined with increased knowledge generation, engagement in RU impacts the quality of care delivered as it can validate existing nursing knowledge and practices, facilitate change and innovation that leads to improved patient outcomes, encourage use of critical thinking, promote reflective practice and facilitate safe and effective care. Moreover, RU can enhance the professional image of nursing, expand the nursing professions' scientific knowledge base and provide evidence of professional accountability (Barnsteiner, Reeder, Palma, Preston \& Walton, 2010; Brown, Wickline, Ecoff \& Glaser, 2009; Corchon, 2010; Estabrooks, 1998; Melnyk, Fineout-Overholt, Gallagher-Ford \& Kaplan, 2012; Mensik, 2011; Myers \& Meccariello, 2006; Parkosewich, 2013; Rycroft-Malone et al., 2004; Tod, Palfreyman \& Burke, 2004; Wallis, 2012). In addition to benefits for the nursing profession, RU is also valuable to researchers as it can validate research efforts, encourage researchers to continue discovering new knowledge and also highlight the need for research in other areas (Burns \& Grove, 2009; Loiselle, Profetto-McGrath, Polit \& Beck, 2007; McEwen \& Willis, 2011). The value also extends to the organization as engaging in the use of research enables the delivery of high-quality care that is based on scientific research, improves patient outcomes, provides data on cost-effectiveness and results in nursing staff who are proactive and professionally satisfied in the delivery of care (Brown et al., 2009; CNA, 2010; Melnyk et al., 2012; Mensik, 2011; Tetroe, 2007; Wallis, 2012).

Despite findings to support the effectiveness of using research in practice, there is evidence to suggest that the disparity between knowledge generation, research in particular, and its utilization persists (Hutchinson \& Johnston, 2006; Roxburgh, 2006). One source suggests that it takes 17 years for research to reach practice, and only 14\% of this research is actually used in 
clinical practice (Balas \& Boren, 2000). This suggests that there is a critical gap between research and practice, and even when research is used, there is time delay from generation to use. Considering that the nursing profession aims to improve the health and well-being of members in society based on a specific knowledge and skill base, the limited utilization of research has the potential to impact the delivery of quality care. According to the College of Nurses of Ontario (2014a), the delivery of quality care is achievable when nurses use evidence obtained from a variety of sources to guide their clinical practice. The word evidence constitutes any form of knowledge that can be used by nurses and it includes research, tradition, authority, clinical experience, intuition, trial and error, patients' views and beliefs and findings from quality improvement initiatives (Burns \& Grove, 2009; Loiselle et al., 2007; McEwen \& Willis, 2011). Among the sources mentioned, research is the most underutilized among nurses, but also the most important to guide decisions about practice change and subsequently increase quality of care, and for this reason it will be emphasized in this study (Estabrooks, 2003; Loiselle et al., 2007; Melnyk et al., 2012).

With the importance of RU assisting healthcare professionals to provide quality care, it becomes critical to explore the factors that may be impeding RU (Burns \& Grove, 2009). The significance of this study lies in exploring the factors that influence RU among nurses and how these can be addressed to promote RU. In the remainder of this chapter a brief summary of the research on individual attributes of nurses and those related to the nature of research and its impact on RU will be presented. Furthermore, the need to explore the organizational factors that may impact RU will be discussed. The concept of organizational capacity will be introduced and a case made for the importance of this study. 


\section{Exploration of Individual Attributes}

Traditionally, the focus of investigations examining factors that impact RU have centered around individual attributes of the nurse such as years of experience, level of education, attitude toward research use and knowledge of acquiring and using research (Ketefian, 1975; ScottFindlay \& Golden-Biddle, 2005; Cadmus et al., 2008; Estabrooks, 2003; Kocaman, Seren, Lash, Kurt, Bengu, \& Yurumezoglu, 2010; Mills, Field \& Cant, 2010; Melnyk et al., 2012; Myers \& Meccariello, 2006; Rycroft-Malone et al., 2004; Scott-Findlay \& Golden-Biddle, 2005; Tod et al., 2004). In an effort to improve RU among nurses, the development and implementation of strategies have been driven by individual attributes that were thought to contribute to decreased RU among nurses. The focus of these strategies has been to address modifiable attributes such as attitude toward research use and education regarding the technical components of RU (acquiring, critiquing and applying research). Various strategies that have been used to equip nurses with the knowledge and skills required to engage in and improve the use of research include: nursing research committees (Barnsteir et al., 2010), evidence-based projects or fellowships (Barnsteir et al., 2010; Hauck, Winsett \& Kuric, 2013; Melnyk et al., 2012), journal clubs (Drenkard, 2013; Krugman, 2010; Mensik, 2011; Parkosewich, 2013), research champions or mentors (Hauck et al., 2013; Melnyk et al., 2012; Parkosewich, 2013), collaboration with academic institutions (Drenkard, 2013; Missal, Schafer, Halm \& Schaffer, 2010), research day and/or symposium (Hauck et al., 2013; Kocaman \& Lash, 2011; Krugman, 2010; Melnyk et al., 2012) and financial resources (Kocaman \& Lash, 2011).

Results examining the effectiveness of the strategies have revealed that while there is benefit to using such strategies, their level of impact is questionable as many nurses continue to be disengaged with RU. Moreover, there are other factors that have the potential to influence the 
application of research in practice that must be explored. A landmark systematic review by Estabrooks, Floyd, Scott-Findlay, O'Leary and Gushta (2003), including an updated version by Squires, Estabrooks, Gustavsson and Wallin (2011) explored the influence of individual nurses' attributes on RU and concluded that with the exception of beliefs and attitudes, there is limited evidence to suggest individual attributes impact RU. Upon analysis, this may suggest that either the strategies themselves were not effective or individual attributes may not sufficiently explain variability in RU (Corchon, 2010; Kocaman et al., 2010; Rycroft-Malone, 2008; Scott-Findlay \& Estabrooks, 2006). These results facilitated a shift in research to being exploring other factors that may be contributing to decreased RU among nurses.

\section{Nature of Research}

In addition to individual attributes of nurses, factors related to the nature of research have also been analyzed for their impact on the use of research. More specifically, findings have explored practitioners' access to relevant research in a timely manner and also use of jargon in research (Lavis \& Catallo, 2013; Lavis, 2006; Lavis, Davies, Denis, Golden-Biddle \& Ferlie, 2005). Having access to research when it is needed, and more specifically an inability to access research in a timely manner was found to limit its use among policymakers (Lavis \& Catallo, 2013; Lavis et al., 2005). In addition, healthcare managers and policymakers in Lavis and colleagues' (2005) systematic review also stressed the importance of having research that was relevant to their context and the decisions that needed to be made. The findings of the systematic review also suggested the need for pre-synthesized evidence (e.g., systematic reviews) which is formatted in a short and easy to read manner. While important, the factors related to the nature of research also do not account for the organizational factors which impact a nurse's use of research in practice. 


\section{Organizational Factors}

Researchers have suggested that although strategies targeting the individual attributes of nurses are important, they often do not account for the environment in which the nurse is employed namely, the organization (Brown et al., 2009; Cadmus et al., 2008; Corchon, 2010; Kocaman \& Lash, 2011; Melnyk et al., 2012). More specifically, strategies that target the individual attributes of nurses fail to consider how the organization may or may not contribute to RU. One of the primary reasons that it is critical to explore the organization is because the individual nurse cannot be separated from the administrative, political, organizational and social factors that surround practice (Cadmus et al., 2008; Corchon, 2010; Kocaman \& Lash, 2011; Melnyk et al., 2012; Myers \& Meccariello, 2006; Rycroft-Malone, 2008; Scott-Findlay \& Golden Biddle, 2005). Each of these factors has the potential to impact RU and associated behaviours among nurses. Lack of time, resources, access to mentors, organizational and leadership support, adequate budget to train professionals and incentives are examples of the top organizational factors known to influence nurses' use of research (Brown et al., 2009; Cadmus et al., 2008; Estabrooks, 2003; Gerrish et al., 2012; Hauck et al., 2013; Kitson et al., 2011; Melnyk et al., 2012; Pravikoff, Tanner \& Pierce, 2005; Sandström, Borglin, Nilsson \& Willman, 2011; Squires et al., 2012; Wilkinson, Nutley \& Davies, 2011).

Each of the components is a part of the administrative, political, organizational and social context that surrounds a nurse's practice and can influence his or her decision to engage in RU. There is research suggesting these components can act as a facilitator or a barrier to using research, resulting in variability in context and making some organizations more conducive to supporting RU than others (Chau, Lopez \& Thompson, 2008; Corchon, 2010; Kocaman \& Lash, 2011; Melnyk et al., 2012; Rycroft-Malone, 2008; Scott-Findlay \& Golden Biddle, 2005). 
Similar to this notion, Rycroft-Malone (2008) and Scott-Findlay and Golden Biddle (2005) contend RU is becoming widely recognized as a contextual phenomenon, which varies by settings. In this sense, it becomes important to explore organizations and how these settings support nurses in their RU efforts. Empirical literature exploring the organization and the factors that make a particular environment better suited to support RU over others constrains is limited and thereby hinders nurses' engagement in RU and the provision of care that is based on current and scientifically sound research. In addition, the lack of research in this area inhibits practice change that may lead to improved patient care and outcomes.

Organizational capacity. One factor that can produce variability in an organization's ability to create an environment that supports research use is organizational capacity. Although not completely novel in the field of nursing, exploration of organizational capacity is rather limited. In one of the earliest research studies about organizational capacity, Rutledge and Donaldson (1995) sought to determine the influence of the Orange County Research Utilization in Nursing (OCURN) project on an organization's capacity to promote research use. Findings of this study highlighted that when nurse executives focused on incorporating RU into the organization's mission and vision, policy, budget and strategic goals and when clinical nursing leaders focused on coordinating and developing RU programs, nurses were more engaged with the use of research and departments reported being more research oriented. This earlier study highlights the importance of multiple components within an organization which might contribute to nurses' engagement with RU.

More recently, a team of researchers from the SUPporting POlicy relevant Reviews and Trials (SUPPORT) project developed a series of articles intended to support individuals responsible for making decisions about health policy and programs (Lavis, Oxman, Lewin \& 
Fretheim, 2009). Stemming from the evidence-informed decision-making movement, the series aims to promote and support evidence-informed health policymaking and also offers users a set of tools that can be used to support the use of research. Of particular importance in this study is the discussion surrounding an organization's capacity to support evidence-informed policymaking, which includes promoting the use of research in practice. Moreover, the series outlines components of organizational capacity that are important for any organization to consider when exploring support for research use, regardless of the setting.

The SUPPORT team suggests that an integral aspect of improving organizational support involves an organization's assessment of their capacity to use and support research use among professionals (Oxman, Vandvik, Lavis, Fretheim \& Lewin, 2009). In addition, Oxman and colleagues' (2009) introduce a self-assessment tool that can assist organizations assess and improve their capacity to use research. While the authors do not provide a formal definition of organizational capacity, they suggest that if organizations want to improve the degree to which decisions are based on research evidence, sufficient capacity is needed. Oxman and colleagues (2009, p. 3) explain “... [sufficient capacity] is necessary for acquiring research when it is needed, critically appraising it, using it to inform decisions..." Therefore, organizational capacity is defined in this study, building upon Oxman and colleagues (2009) discussion, as the extent to which organizations have the necessary conditions, resources and processes to support the use of research.

Components of organizational capacity. The self-assessment tool, which is called the Organizational Capacity to Support the Use of Research Evidence to Inform Decisions Instrument (henceforward called the Organizational Capacity Instrument), is organized into seven sections, each corresponding to a component of organizational capacity (Oxman et al., 
2009). The seven components include: (a) organizational culture and values that support the use of research, (b) setting priorities for obtaining research evidence to inform decisions, (c) obtaining research evidence to inform decisions, (d) assessing the quality and applicability of research and interpreting the results to inform decisions, (e) using research evidence to inform recommendations and decisions, (f) monitoring and evaluating policies and programmes and (g) supporting continuing professional development (Oxman et al., 2009). Each of these seven components is critical in supporting use of research and also assisting organizations to explore how and if they are facilitating research use. This tool and the corresponding components were used to operationalize organizational capacity in this study.

\section{Organizational Capacity and Senior Nursing Leaders}

Research focusing on the importance of senior nursing leaders highlights their influence on components that impact an organizations' capacity to support research use (Gerrish et al., 2012; Kitson et al., 2011; Melnyk et al., 2012). There is increasing recognition that senior nursing leaders play a key role in cultivating an environment that facilitates RU among nurses and that they possess the power and authority to address organizational capacity to support RU (Gerrish et al., 2012; Hauck et al., 2013; Melnyk et al., 2012; Parkosewich, 2013; RycroftMalone, 2008; Sandström et al., 2011; Squires et al., 2012; Wilkinson et al., 2011). For this reason it was imperative to include senior nursing leaders when studying organizational capacity and RU as they are viewed as mediators between nurses and senior management, are expected to embody the organization's mission, vision and values and to create appropriate capacity to promote RU among nurses (Gerrish et al., 2012; Hauck et al., 2013; Melnyk et al., 2012; Parkosewich, 2013; Rycroft-Malone, 2008; Sandström et al., 2011; Squires et al., 2012; 
Wilkinson et al., 2011). In addition, individuals with these roles have an understanding of systems level factors that influence an organization's capacity to support research use.

Estabrooks (2003) suggested lack of support from leaders was a major barrier to promoting RU among nurses and proposed that there is often confusion as to whether leaders understand their role in shaping an environment that promotes RU among nurses. Other authors have also suggested that leaders are critical in establishing an environment that supports RU (Gerrish et al., 2012; Hauck et al., 2013; Melnyk et al., 2012; Sandström et al., 2011; Squires et al., 2012; Wilkinson et al., 2011). The support of leaders is critical not only because they provide vision and support for RU and associated activities, but because they are in a key position to allocate the necessary resources required to use research such as, time, education, funding, networks and research access (Gerrish et al., 2012; Hauck et al., 2013; Melnyk et al., 2012; Sandström et al., 2011; Squires et al., 2012; Wilkinson et al., 2011). Without the support of leaders, access to resources for nurses would limit capacity building and motivation to engage in RU at the individual level.

While the importance of studying organizational capacity and support for RU with senior nursing leaders has been established, the amount of research in this area is minimal (Corchon, 2010; Kocaman \& Lash, 2011; Squires et al., 2012). Eliciting data regarding the facilitators and barriers to RU, from the leaders' perspective, is also essential as leaders have an understanding of the organization and how the system impacts RU. In addition, leaders also hold key information about practice level factors that impact RU among nurses. Therefore, directing attention towards developing an organizational-level understanding of RU among senior nursing leader is imperative as organizations can assess their capacity to support RU. In addition, 
exploration of organizational capacity might also assist organizations identify areas of improvement and how these areas can be addressed to support and promote RU among nurses.

Senior nursing leaders. Participants in this study were limited to the nursing profession. For the purpose of this study, the term "senior nursing leader" referred to individuals in management, corporate and executive positions in health care settings. Senior nursing leaders are critical in influencing organizational capacity to support research use. An individual with power and authority but also with advanced knowledge and understanding of research use is needed to create an environment conducive to RU (Rycroft-Malone et al., 2004).

\section{Statement of the Problem}

The majority of nursing research exploring the organization has been conducted in settings outside of Canada including the United States of America (USA), United Kingdom (UK) and Europe. Although findings from studies conducted in these settings have implications for the future of RU in nursing, it is important to recognize the context in which this research was conducted. Because the healthcare system and nature of nursing practice varies between countries and within provinces, generalizability is a concern. It is especially important to conduct research that is context-appropriate. Furthermore, the College of Nurses of Ontario (2002) has outlined that the use of research is a professional responsibility of practicing nurses. Thus far, there has been minimal exploration of organizational capacity and RU within Canada and more specifically Ontario, thus emphasizing the need for Canadian research.

Although the importance of using research in practice has been established, nurses continue to be disengaged with this practice (Breimaier, Halfens \& Lohrmann, 2011; Cadmus et al., 2008; Melnyk et al., 2012). Inconclusive findings from research examining individual attributes of the nurse have facilitated a shift to exploring the organization and how 
organizational factors have the potential to act as facilitators or barriers to RU. Furthermore, although the role of senior nursing leaders has been recognized as influential, their insight regarding organizational capacity to support RU and the facilitators and barriers to RU have not been well explored.

\section{Purpose of the Study}

The purpose of this study was to examine the presence of components of organizational capacity which support research use, as identified by senior nursing leaders within their respective healthcare organizations in Ontario. In addition, this study explored the facilitators and barriers to research utilization.

\section{Significance}

The importance of research utilization and nursing professionals engaging in the use of research is critical. With a growing amount of research available in health and nursing, it is a professional expectation that research evidence be incorporated into practice (Canadian Nurses Association, 2010; College of Nurses of Ontario, 2014a; International Council of Nurses, 2012). The position statements and professional standards of nursing organizations suggest it is every nurse's responsibility to engage in the use of research. This engagement can be at any part of the research continuum; for example nurses can be involved in the generation or utilization of research, but regardless of the means, the responsibility to participate in RU exists. Whether nurses are consumers or actively involved in conducting research, they have a personal and professional responsibility to consider these recommendations. Increasing patient and family involvement and expectations for care also necessitate that nurses be able to support that their decisions are based on the best available evidence, further reinforcing the importance of RU. 
Despite the documented benefits of research utilization, limited involvement among nurses is persistent (Breimaier et al., 2011; Cadmus et al., 2008; Melnyk et al., 2012). As discussed previously, research has illustrated inconclusive findings regarding the impact of individual attributes on RU. However, a shift in focus to the organization has introduced components that may serve to increase understanding and possibly explain poor rates of research utilization among nurses. More specifically, assessment of organizational capacity is important as it can offer information about how organizations support research use. Research utilization is a complex process that has not been well explored with senior nursing leaders in Ontario. This study will be a first step in identifying the extent to which components of organizational capacity, which support research use, are present in various organizations in Ontario. Furthermore, because senior nursing leaders are important for creating and sustaining an organization that participates in RU and given that their role has been recognized as central to shaping/supporting organizational capacity, it becomes important to include leaders in research efforts. 



\section{Chapter 2: Literature Review}

In this chapter, the literature on organizational capacity, the components of organizational capacity and research utilization (RU) is synthesized and analyzed. Following a description of the literature search strategy, a summary of the literature regarding RU among nurses is provided. Thereafter, literature that examines the impact of individual attributes and explains why a shift in focus on the organization was needed is summarized. This chapter culminates with a synthesis and critique of the literature on the various components of organizational capacity and the gaps in the literature.

\section{Literature Search Strategy}

The literature review was an iterative process that was conducted by searching the following databases: Cumulative Index of Nursing and Allied Health (CINAHL), Medline, ProQuest Nursing \& Allied Health Source, Business Source Elite and Canadian Business and Current Business Affairs Complete. Search terms included: organizational capacity, research utilization, research use, capacity, organization* and definition. The following limits were applied: articles published in the last 10 years (2004-2014), written in English and peerreviewed. Although the search produced some results, it was evident that the conceptualization of organizational capacity was fairly underdeveloped as there were very few articles that focused on this concept (see Appendix A). While there was some discussion about components of the organization, they were discussed in relation to the individual attributes of nurses and their impact on RU. Therefore, the focus of the majority of the articles was on individual attributes.

Consequently, a second search was conducted to capture specific information about the organizational components of RU. The second search involved six additional searches in three databases and one new search in the Cochrane Methodology Register. Various terms, including 
nursing practice, evidence-based, organizational culture, context, evidence-based nursing, and nurs*, were used to locate relevant literature and over 1, 500 articles were identified. The publication language, date and type limiters helped narrow the search results (see Appendix B).

A majority of the literature focused on the individual attributes of nurses that impact RU. Therefore, due to difficulty locating literature on organizational capacity and other organizational components, the reference lists of the limited but relevant articles were also hand searched to locate additional articles. In addition to searching reference lists, the profiles of individual researchers, authors and experts in RU, including Carole Estabrooks, Maureen Dobbins and Anita Kothari, were also searched for publications that could contribute to an understanding of the components of organizational capacity. This strategy also produced limited results.

The selection of articles involved an initial title and abstract screening process, after which, chosen articles were retrieved full text and reviewed to determine their purpose, scope and focus on RU and more specifically the organization. Articles were included if they: explored research use, focused on organizational capacity or a component of it entirely or in parts and involved nurses and/or senior nursing leaders. Although nursing literature was preferred, research from other disciplines was included, if the article was relevant to organizational capacity or research utilization. The search revealed limited focus on organizational capacity or its components.

Results of the search. From the initial search, a total of eight articles were selected in order to gain a better understanding of organizational capacity and its components. However, because a second search was warranted, this led to a final total of 39 articles included in this review. Of the total articles, more than half $(\mathrm{n}=23)$ were empirical, two were reviews, and 14 were non-empirical in nature. Of the 23 empirical articles, $65 \%(\mathrm{n}=15)$ were quantitative, mostly 
cross-sectional in nature and there were no experimental studies. Two of the quantitative articles were secondary analyses. Among the non-empirical articles there were theoretical and discussion papers. Of the empirical literature, the sample of articles included: nurses $(n=12)$, various healthcare professionals $(n=4)$, leaders exclusively $(n=3)$, a mix of nurses and leaders $(n=3)$ and other $(n=1)$. A majority of research involved the staff nurse and very little attention has been directed towards senior nursing leaders.

\section{Quality of Included Literature}

As outlined above, 14 of the 39 sources included in this review were non-empirical in nature. These sources were included as they presented the opinions of experts in the fields of RU, evidence-based practice and evidence-informed decision-making. Furthermore, the inclusion of sources such as these point to areas where empirical research is needed. The methodological quality of the remaining 25 sources is discussed below.

Qualitative literature. A total of eight qualitative studies were included in this review of which three were case studies (Gerrish et al., 2012; Wilkinson et al, 2011; Hamel \& Schrecker, 2011) one each hermeneutics (Salmela, Eriksson \& Fagerström, 2013), ethnography (Jokelainen, Jamookeeah, Tossavainen \& Turunen, 2011) and phenomenology (Scott \& Pollock, 2008) and two did not specify a type of qualitative methodology (Ellen et al., 2013; Shea et al., 2014). All but one study (Shea et al., 2014) justified the type of research design chosen. Six studies used purposive sampling, one used key informants identified by the organization (Hamel \& Schrecker, 2011) and one did not specify sampling details (Shea et al., 2014). Sample size ranged from six (Hamel \& Schrecker, 2011) to 157 (Ellen et al., 2013) participants, and only Hamel and Schrecker mentioned saturation. Data were primarily collected through semistructured interviews $(n=3)$, a combination of multiple methods such as interviews, document 
reviews and participant observation $(n=3)$ and structured interviews $(n=2)$. Some sources discussed development of the interview guide as based on scoping reviews, literature reviews and various frameworks.

A majority of the studies used a particular framework to conduct data analysis $(n=5)$, two others used data analysis software and provided details on how coding was done, while one study used a list of pre-identified codes which were altered as new themes emerged (Shea et al., 2014). Only two studies reported validating emerging themes and coding schemas with participants (Hamel \& Schrecker, 2011; Scott \& Pollock, 2008). Furthermore, only three studies mentioned the use of note taking for self-reflection during the research process, yet this is an important component of qualitative research. As the level of detail regarding the research process varied among studies, this had implications for determining the trustworthiness of the qualitative studies. For example, credibility in most studies was limited as researchers failed to complete member checking with participants and many researchers did not discuss completing selfreflection during the research process (Creswell, 2007). Furthermore, transferability of research findings was not discussed within many qualitative studies (Creswell, 2007). In addition, dependability of the research was lacking as the level of detail regarding research design and its implementation varied among studies (Creswell, 2007).

Quantitative literature. Of the 15 quantitative studies, 13 used a descriptive, crosssectional survey design and two studies identified using a comparative (Hauck et al., 2013) and correlational design (Melnyk, Fineout-Overholt, Giggleman \& Cruz, 2010). Six studies sampled from one acute care hospital in a given state within the United States, two studies sampled from multiple hospitals in the United States (Cadmus et al., 2008; Estrada, 2009) and four studies sampled from acute care hospitals in other countries (Australia, Austria, Turkey and England). 
Of the four international studies, two sampled from more than one hospital (Bonner \& Sando, 2008; Gerrish, Ashworth, Lacey \& Bailey, 2008). The remaining three studies sampled from registered nurses across Hong Kong (Chau et al., 2008) and the United States (Melnyk et al., 2012; Pravikoff et al., 2005) using data from their respective national nursing organization. The participants from the studies included in this review were primarily front-line staff nurses and limited to those practicing in acute care hospitals outside of Canada. These factors are important to highlight as they limit the ability with which the results can be generalized to settings in Canada, outside acute care hospital settings and to samples beyond those identified in the studies. Over $70 \%(n=11)$ of the studies used convenience sampling, one used purposive sampling (Melnyk et al., 2010) and three used a form of random sampling (Chau et al., 2008; Melnyk et al., 2012; Pravikoff et al., 2005). A majority of the studies had response rates between 30-39\% (n=5) and 50-59\% (n=4) and one study did not specify a response rate (Melnyk et al., 2010). Burns and Grove (2009) describe a response rate of $73 \%$ as somewhat low; yet there was only one study which had a response rate of $63 \%$ suggesting the rates were on the low end. These are limitations of the studies that may affect representativeness of the samples to the target population and subsequently limit ability to generalize the findings (Burns \& Grove, 2009). While response rate is one element that is important to consider, an adequate sample size is also required to do statistical comparisons in descriptive studies. Sample size estimation was only reported by one source, and this was done after the study was completed (Gerrish et al., 2008). However, sample size in the included studies were fairly large and ranged from under $250(\mathrm{n}=2)$, 251- $500(n=5), 501-1000(n=3), 1001-1500(n=4)$ and one study had over 3000 participants.

Of the 15 studies, nine studies described using reliable and valid instrument(s) and two used a research-developed tool that was tested (Pravikoff et al., 2005; Thiel \& Ghosh, 2008). 
Other studies used tools without established reliability and validity (Bartlet et al., 2011; Cadmus et al., 2008) or were mixed in which some tool(s) were evaluated and other(s) were not (Breimaier, Halfens \& Lohrmann, 2011; Chau et al., 2008). These factors impact the confidence in the results of some studies and stress the importance of using tools with some preliminary psychometric testing. This literature review also revealed the lack of instruments that focused on measuring organizational capacity exclusively in relation to RU. These points highlight the need for more research with relevant and validated instruments.

Data analysis techniques involved the use of statistics to: examine relationships (Pearson's r, Spearman's rank order correlation), predict (regression analysis) and examine causality (Chi-square test, t-test and one way Analysis of Variance) (Burns \& Grove, 2009). Some of the variables being used to analyze differences in the studies included: participating vs. not participating in an educational series, demographic information, types of positions, subscales of instruments, nurse characteristics, barriers and facilitators to RU, time and level of involvement in research activities. The most common statistical test used was the Chi-square and two studies solely used descriptive statistics. While the studies reviewed were descriptive in nature, the larger sample sizes may have permitted enough variability in the sample to facilitate the use of multiple different types of statistical methods. In addition, three studies included qualitative data, for which content analysis was used.

Systematic reviews. Both the systematic reviews were guided by research questions, outlined inclusion and exclusion criteria, provided search strategies, conducted duplicate screening and quality appraisals, used a consensus process for discrepancies in screening and presented characteristics of the included studies (Estabrooks et al., 2003; Sandström et al., 2011). While Estabrooks et al. (2003) used seven databases and included 22 articles in their review of 
individual attributes of nurses that impact RU; Sandström et al. (2011) used three databases and included seven articles to explore the influence of leaders on the process of evidence-based practice. The difference in yield may be due to the topic of interest in the reviews.

Although both reviews assessed the quality of articles, Sandström et al. (2011) used standardized, well-known tools to conduct appraisals but did not report the quality of each study individually and Estabrooks et al. (2003) used a researcher developed tool and classified each of the articles into strong, moderate and weak categories. However it is important to note that neither of the reviews calculated inter-rater reliability scores among the reviewers conducting the quality appraisals. Overall, the reviews were well done, the results were clearly presented, they included quantitative and qualitative studies and they are rated high on the hierarchy of evidence.

\section{Synthesis of the Literature}

Individual attributes and their impact on research utilization among nurses. In a

seminal article, Estabrooks and colleagues (2003) summarized 30 years of research in the field of RU by identifying six categories of potential individual attributes that were thought to influence research use. The categories of attributes included: "beliefs and attitudes, involvement in research activities, information seeking, professional characteristics, education and other socioeconomic factors" (Estabrooks et al., 2003, p. 506). Estabrooks and colleagues (2003) concluded that with the exception of attitudes towards RU, there was little to suggest that any of the individual attributes influenced RU. In addition to exploring specific individual attributes, some sources investigated nurses' use of research, information seeking behaviors and sources of information sought. While the latter two topics are not a direct measure of RU, the findings reveal the types of sources sought when nurses need information. More specifically, these 
findings, may shed light on how willing nurses are and if there is actual use of research when information is needed.

From the sources that reviewed nurses need to seek information, two sampled nurses from acute care hospitals in the United States (Cadmus et al., 2008; Thiel \& Ghosh, 2008), and another involved a sample of randomly selected nurses from different geographical locations in the United States (Pravikoff et al., 2005). The findings from two sources revealed nurses needed to seek information several times a week (Cadmus et al., 2008; Pravikoff et al., 2005) and nurses in another study reported needing to seek information daily or many times a day (Thiel \& Ghosh, 2008). While the sample size of these sources ranged from 120 to over 3000 participants, these results indicated that nurses seek information on a regular basis; however, what is important to consider is the source of the information.

When exploring the most common sources of information, it was reported that nurses relied heavily on peers/colleagues (Cadmus et al., 2008; Gerrish et al., 2008; Pravikoff et al., 2005; Thiel \& Ghosh, 2008), the Internet (Cadmus et al., 2008) and personal experience (Gerrish et al., 2008). The results of this literature review revealed that the use of bibliographic databases, journal articles and research reports as sources of information among nurses was very limited (Pravikoff et al., 2005; Thiel \& Ghosh, 2008). In addition to limited use of the sources mentioned above, use of libraries and librarians when nurses needed information was also revealed to be minimal (Cadmus et al., 2008; Pravikoff et al., 2005; Thiel \& Ghosh, 2008). The sources of information utilized by nurses in times of need reveal a critical gap in the use of research.

Only five articles specifically examined the use of research among nurses. In a crosssectional study, $77.4 \%(n=767)$ of nurses reported never/seldom using research results in the past year (Breimaier et al., 2011). Similarly, 43.7\% $(n=1,490)$ of nurses in Cadmus and 
colleagues' (2008) study reported never using research. Melnyk et al. (2012) asked nurses about their colleagues' use of research, of which, only 350 (34.5\%) agreed or strongly agreed that their colleagues consistently implemented research in their practice (Melnyk et al., 2012). And in one correlational study with 58 nurses and health care professionals, findings suggested that although participants' beliefs about evidence-based practice and their ability to implement research in practice were rated moderate to strong, their level of research implementation was low (Melnyk et al., 2010). In the only study with nurses employed in rural and remote areas, $63.6 \%(\mathrm{n}=222)$ of participants reported not having any research involvement, specifically reading journal articles either on paid or unpaid time, in the last two years (Bonner \& Sando, 2008). Three studies were conducted with nurses in hospital settings (Breimaier et al., 2011; Cadmus et al., 2008; Melnyk et al., 2010), one from rural and remote areas (Bonner \& Sando, 2008) and another with nurses in a variety of sectors and geographic areas (Melnyk et al., 2012).

While the results discussed above indicate that level of nurse engagement in research was generally low, variability in levels of disengagement was apparent. One of the reasons may be related to differences in settings and sample size of the studies. In addition to differences in how the health care system is organized in these various settings, differences in the type of organization (e.g. hospital vs. community) and rural versus urban settings may also have an impact on the types of resources professionals have and their access to research may vary as a result. For example, resources and access to research for nurses in a rural and remote setting may be limited when compared to nurses in an urban, acute care hospital. Another reason for this variability might be related to differences in how each of the studies conceptualized and collected data on research use. While some studies used subscales on validated tools, others used 
researcher-developed Likert-based scales. These differences might impact how participants interpret and respond to questions and limit the ability to make comparisons across studies.

\section{Organizational Capacity}

As evidenced by a review of the literature, if individual attributes of nurses fail to completely or consistently account for disengagement in RU, alternative factors must be explored. Authors suggest the factors within the organization, which is sometimes referred to as the setting, environment or context, also need to be examined (Corchon, 2010; Ellen et al., 2013; Kocaman et al., 2010). One such factor, which was introduced in the previous chapter and is gaining interest amongst those investigating RU, is organizational capacity. It is important to explore an organizations' ability to support research use, as it is essential to identify contextspecific factors that may influence the use of research. Organizational capacity has not been explored extensively in the nursing discipline or in relation to RU; however the individual components of organizational capacity have been explored. The remainder of this chapter will include highlights of the literature that pertains to the components of organizational capacity.

Organizational culture and values and setting priorities to obtain research. The first two components of organizational capacity are concerned with the importance an organization places on RU and how this impacts support for RU. The following themes will be discussed in this section: organizational culture, mission and vision and leadership support.

Organizational culture. Organizational culture and the role it plays in supporting RU is perhaps one of the most studied components. Experts such as Rycroft-Malone (2008) and Scott and Pollock (2008) have stated that researchers should focus on the conditions under which nurses are expected to engage in RU. Both Rycroft-Malone (2008) and Scott and Pollock (2008), relied on Schein's (1992) description of organizational culture as the artifacts, values and 
assumptions present in an organization. The culture that is present within an institution is thought to directly impact nurses' engagement in RU. In their discussion papers, Corchon (2010) and Kocaman and Lash (2011) posit that culture impacts RU because it directly relates to the attitudes professionals have about RU and how these attitudes translate into displayed behaviors. In another discussion paper, Squires and colleagues (2012) suggest nurses often draw on the culture within their unit and organization when deciding how to act or behave or when formulating their beliefs. These authors propose that nurses will often change their attitudes and subsequent behaviour to be reflective of the shared beliefs and attitudes of others on the unit. While these points provide support for the importance of creating a culture which values and supports RU, many of these perspectives have not been empirically validated, limiting the confidence with which conclusions can be accepted.

Two studies that explored organizational culture empirically and highlighted the importance that culture plays in supporting RU among nurses were located. In a descriptive, cross-sectional survey of 458 nurses from an academic medical center in California, two of the top four barriers to RU were related to support from the organization and the culture of using research within the organization (Brown et al., 2009). The authors reported that when research use was built into the culture of a particular organization, it was seen as a facilitator to RU (Brown et al., 2009). In another descriptive cross-sectional study, Thiel and Ghosh revealed nurses' attitude toward research use “...was strongly correlated with unit culture... and moderately correlated with organizational culture" (2008, p. 187). While this research illustrates that nurses may be more receptive to and engage in RU if organizations create a supportive culture, both the studies were conducted in a single hospital in the United States and generalizability is a concern. 
Various elements of the organization were also identified as barriers to RU in a quantitative study among 275 nurses in a Magnet hospital in Massachusetts (Karkos \& Peter, 2006). Results from this study revealed four out of the top five barriers, identified using the BARRIERS tool (Funk et al., 1991), were related to the organization. Furthermore, 39\% of nurses revealed a supporting and encouraging environment would facilitate research use (Karkos $\&$ Peter, 2006). When asked to respond to an open-ended question about what prevents professionals from implementing evidence-based practice, the second most common response was "organizational culture, including policies and procedures, politics, and a philosophy of that is the way we have always done it here" ( $\mathrm{n}=123)$ (Melnyk et al., 2012, p. 414).

Although these findings point to the importance of having an organization that supports RU through its' culture, values and policies, they should be interpreted with caution as the results do not provide strong support, given that the proportion of participants who responded to the questions above when compared to the total sample was relatively small in both studies. This also supports the need to continue to examine the importance of organizational culture, values and policies on RU further. Furthermore, Karkos and Peter (2006) sampled from a Magnet hospital and these organizations understand the importance of and support nursing research and are often recognized for the high quality patient care they provide (American Nurses Credentialing Center [ANCC], 2013). Because these organizations provide significant amounts of support for nursing research, have more supportive organizational cultures and are granted Magnet status by the ANCC, these results may not be comparable to other settings.

While an organization's culture and values must be supportive in order to facilitate RU, nurses' values regarding RU are also important to consider as they can also impact an organization. In a descriptive study of 760 nurses, the lack of value for research use was reported 
as the top individual barrier when respondents were asked to rank the top three barriers from a list of 10 (Pravikoff et al., 2005). In addition to being reported as an individual barrier, an organization's perception that nursing staff was not ready to engage in the use of research was also mentioned as an institutional barrier. This highlights that nurses' value for RU may not only have impact on an individual, but also an organizational level. One possible explanation for this finding may be related to the organization's value and support of RU. For example, the sixth and last institutional barrier reported by Pravikoff and colleagues (2005) was the organization's perception that RU was not achievable in the "real-world". If the organization itself does not place importance on using research or believe it is possible to implement, nurses are less likely to engage in the practice (Penz \& Bassendowski 2006; Pravikoff et al., 2005; Tod et al., 2004). These elements create a cyclic situation in which nurses report lack of value of research and are not engaging in RU due to unsupportive environments, yet the organization perceives lack of nurse interest as a barrier to RU and also doubt the practicality of RU.

The findings discussed above have noteworthy implications for engaging nurses in RU as it can be argued that if the organization itself does not value, support or believe that RU is practical, it can be challenging to elicit support among nurses. Although the findings reported above are meaningful, it is important to note these studies sampled mainly front-line staff nurses and did not seek the perspectives of senior nursing leaders. In addition, only one study (Karkos \& Peters, 2006) used a validated tool to collect data about barriers to RU; however this tool has a strong focus on individual barriers. The other studies relied on open-ended questions (Melnyk et al., 2012) or had participants select from a list of barriers (Pravikoff et al., 2005), as there are no tools that explore organizational supports for RU exclusively. Finally, none of the studies were from Canada and this has implications for generalizability of findings, especially when 
concerned with a component such as organizational culture, which is context dependent.

Mission and vision. An organization's mission and vision statements and how these indicate support for RU are other elements that are important to consider. When organizational documents incorporate and promote the importance of using research, it is communicated as a strategic organizational priority. Furthermore, when RU is incorporated in organizational documents, the expectations that the organization and leaders have of nurses are also more apparent (Thiel \& Ghosh, 2008).

Melnyk and colleagues (2012) reported that when RU was incorporated in the mission and vision and nurses perceived their culture as supportive of RU, their beliefs about using research and actual use of research was higher. Considering that a nurse's attitude is an individual attribute that might influence RU, it may be beneficial for organizations to analyze their mission, vision and the overall culture to determine if they might be contributing to negative attitudes (Estabrooks et al., 2003). When nurses from Thiel and Ghosh's (2008) study reported their organization was moderately supportive of RU, the authors suggested that support and commitment to RU, which could impact attitudes, might be enhanced through the mission and vision of an organization. While embedding the importance of RU in the organization's mission, vision and culture was identified as important, the establishment of these elements requires considerable commitment from leaders (Estrada, 2009; Hauck et al., 2013; Melnyk et al., 2012; Rycroft-Malone, 2008; Sandström et al, 2011; Wilkinson et al., 2011).

Leadership support. As introduced previously, leaders are critical to creating a culture that supports and engages professionals in RU. A lack of support from the organization and leaders was mentioned as a barrier to RU in the literature numerous times (Brown et al., 2009; Gerrish et al., 2012; Hauck et al., 2013; Melnyk et al., 2012; Sandström et al., 2011; Squires et 
al., 2012; Wilkinson et al., 2011). In a case study involving 51 interviews with nursing leaders, Wilkinson and colleagues' (2011) findings revealed leaders can support RU by: acting as champions, providing guidance in activities throughout the RU process, and empowering nurses to engage in RU. This suggests that in addition to offering knowledge and skills related to RU, leaders might also have a role in motivating and encouraging nurses to engage in RU.

Nursing leaders from Wilkinson and colleagues' (2011) case study described taking a passive approach when promoting RU among nurses. Leaders emphasized that competing demands, feelings of being overloaded and lack of adequate preparation in supporting nurses often contributed to the passive approach taken by many leaders. While the findings from this case study raise concerns about the variability of leaders' involvement in RU due to working conditions, it is important to note that the authors did not discuss how the sample was recruited, or which type of setting(s) participants were employed in and the methodology does not permit the findings to be generalized to other organizations.

Setting priorities. One of the very few studies that addressed organizational priorities found the presence of other goals with a higher priority was the second highest organizational barrier among pediatric healthcare professionals (Bartlet et al., 2011). The authors highlighted that evidence-based practice must be valued and prioritized as highly as direct clinical care in order to minimize organizational barriers. The authors also suggested efforts to reduce organizational barriers such as this, should be a priority of nursing leaders. While there was a lack of literature on this particular topic, it can be argued that the presence of the factors discussed throughout this chapter would indicate that an organization prioritizes RU.

Obtaining, critically appraising and using research in practice. The next three components of organizational capacity relate to the technical knowledge and skills required for 
RU. Common themes in the literature revealed a lack of the following factors impact nurses' ability to access, appraise and use research: time, resources, availability of mentors, and rewards.

Lack of time. A lack of time to engage in RU was the number one barrier identified in the majority of studies included in this literature review. Even if it was not mentioned as the most influential barrier, a majority of authors reported that nurses mentioned lack of time was a crucial factor that determined their engagement with RU. For example, in a cross-sectional survey of 1023 graduate nurses in an Austrian hospital, the top barrier to RU was reported as lack of time (69.9\%) (Breimaier et al., 2011). In another survey of organizational barriers, 70.5\% of Hong Kong nurses revealed time as a barrier (Chau et al., 2008). Even when comparing these results to nurses in an academic medical center in California, a lack of time to engage in RU was identified as a concern and the participants explained that high demands, a heavy workload along with the growing responsibility of meeting patient needs were major determinants that impeded availability of time to engage in RU (Brown et al., 2009). While nurses from the study by Brown et al. (2009) offered some explanations for lack of time to engage in RU, Cadmus and colleagues (2008) suggested consistent reporting of this barrier points to a deficit in which the organization is ineffectively supporting nurses. While these studies had comparable results, it is important to note the samples only consisted of front-line nurses.

Lack of resources. The provision or lack of resources, which can refer to human, financial and equipment/technical sources, can also impact RU. In a study that explored organizational supports for RU, results of 57 interviews with senior management, library managers, and knowledge brokers revealed an infrastructure that provides staff with the resources to access to research, such as databases, was a facilitator to promoting RU (Ellen et al., 2013). Ellen and colleagues' (2013) study was one of the only Canadian studies in which the 
authors sampled senior leaders from a variety of organizational settings in Ontario and Quebec. As Ellen and colleagues' study (2013) was Canadian and sampled nursing leaders, it was applicable to this present study.

While multiple sources have reported lack of resources as a barrier to RU among nurses (Brown et al., 2009; Cadmus et al., 2008; Gerrish et al., 2012; Kocaman et al., 2010; Pravikoff et al., 2005), some researchers reported that even when nurses are given the appropriate resources, they are not utilized (Gerrish et al., 2008; Thiel \& Ghosh, 2008). One explanation may be that nurses rely on older practices as a means to avoid using research and its' associated change (Tod et al., 2004). Tod and colleagues (2004) also suggested that nurses have the tendency to 'play victims' and create an image that the organization creates obstacles for nurses that prevent them from engaging in RU. Views such as this shift focus from the nurse as the root of the problem to consideration of the organization. The authors argue that this creates a need to explore these factors further and more specifically seek the perspective of those that manage front-line nurses so they can comment on the availability of resources and nurses level of engagement in RU.

Lack of mentors. Another element that was mentioned in the literature was the lack of mentors or experts in the field of RU. Nurses reported mentors who are knowledgeable about accessing, appraising and using research were valuable as they provided support to nurses, promoted their engagement in RU and were also critical to providing education and training around the technical skills of RU (Brown et al., 2009). In a survey conducted among 1, 015 nurses from magnet and non-magnet organizations, only $329(32.4 \%)$ reported that they had access to mentors, it was seventh amongst a list of ten reported facilitators and further analysis revealed that nurses from Magnet designated hospitals reported higher levels of availability of mentors when compared to those from non-Magnet organizations (Melnyk et al., 2012). 
In a discussion paper which outlined a set of recommendation for leaders to implement research-based policies, Squires and colleagues (2012) suggested the use of champions not only supported nurses with RU but also strengthened the unit culture, facilitated the provision of resources and empowered nurses to engage in RU. Another discussion paper by Smith and Donze (2010) suggested mentors should be advanced-practice nurses who have extensive training and skills in evidence-based practice, as their role should center on education and training and removing organizational barriers for front-line nurses. In addition, the authors argue that mentors play a vital role in implementing and sustaining a culture that is built on evidencebased practice. It is important to note both of these sources are discussion papers and essentially the opinions of experts as opposed to findings that have been tested empirically. Therefore, this highlights a need for research in order to validate the points discussed above.

Lack of rewards. Another element that may influence nurses' decision to engage in RU is the lack of rewards offered for involvement in RU. While it might be assumed that rewards take the form of monetary incentives, a literature review that explored the role of leaders in implementing evidence-based practice revealed that many nurses expect their involvement in evidence-based practice to be reflected in performance reviews (Sandström et al., 2011). The authors suggested that acknowledging the use of research in performance reviews contributed to creating a culture where nurses knew research was valued and this also promoted engagement. In a qualitative study about organizational change, Salmela and colleagues (2013) explain the purpose of rewards, which can be small, was to communicate to nurses that their efforts in using research were acknowledged.

Monitoring and evaluating policies and programmes. While literature regarding monitoring and evaluation in relation to policies and programmes was fairly limited, there was 
literature regarding the provision of feedback in relation to the use of research. Cadmus and colleagues (2008) suggest nurses need to be informed about the benefits of using research in practice in order for them to see the value of evidence-based practice. Furthermore, the authors also suggest the need for a culture that illustrates how the use of research is beneficial for the health-care system. In a descriptive study, $20 \%$ of nurses reported a practical application of RU was a facilitator and they also appreciated feedback on how research use improved patient outcomes (Karkos \& Peters, 2006). These findings suggest that nurses want to see others engage in RU and they also wanted to know how and if RU has positive outcomes for patients.

In another qualitative study, Ellen and colleagues (2013) developed an interview guide based on a scoping review that aimed to explore the current supports organizations have in place for evidence-informed decision-making. Of the seven identified domains of support, evaluation efforts was the only domain where participants expressed a lack of support. In a literature review, feedback from managers, with regards to implementing research in practice, was reported as important in three studies as it facilitated skill development, motivated staff and created a sense of acknowledgement and appreciation among nurses regarding their efforts to engage in evidence-based practice (Sandström et al., 2011). These findings highlight a key role for nursing leaders in relation to proving feedback and monitoring RU.

A lack of emphasis on monitoring and evaluation makes it difficult not only to explore the impact of RU on an organizational or macro level, but also limits the feedback that nurses receive on their implementation efforts on a micro-level. This has implications for points that were discussed earlier in which nurses expressed a need to know about the benefits of RU and also being part of an organization that rewards engagement in RU. A survey which aimed to explore the relationship between the characteristics of a 'learning organization' and nurses' 
beliefs regarding evidence-based practice among nurses in six acute care hospitals concluded that the implementation of evidence-based practice required constant evaluation of nurses' practice and its impact on patient outcomes (Estrada, 2009).

Professional development. Another area that is commonly studied is education to support the development of knowledge and skills related to the use of research in practice. In a cross-sectional study that compared perceptions, skills, and activities related to evidence-based practice among pediatric healthcare professionals who attended and did not attend an educational series, lack of training was one of the top three organizational barriers to RU (Bartlet et al., 2011). When asked about the top three individual barriers, both groups expressed lack of skills surrounding the use of and access to databases as well as, searching and critiquing/synthesizing literature but those who attended the educational series did report more involvement and skills related to evidence-based practice. It was interesting to note the overlap in personal barriers between both groups, despite attending the series (Bartlet et al., 2011). The results of this crosssectional study suggest that there may be a need for in-depth training as even those who attended the educational series reported the same barriers as those who did not attend the series.

Other authors also reported lack of (Pravikoff et al., 2005) or inadequate (Bonner \& Sando, 2008; Breimaier et al., 2011) education, knowledge and skills as a barrier for using research in practice. Findings such as these support the need for tailored education that addresses the needs of nurses, not only for the purpose of increasing knowledge but also applying new knowledge to practice. Barnsteir and colleagues (2010) described an evidence-based practice/ research committee at the Hospital of the University of Pennsylvania whose focus was to engage nurses in the use of research through education and mentoring activities. The authors suggested activities such as a journal club, which reviewed and critiqued research articles, and providing 
access to mentors, who were knowledgeable about research use, were positively received by nurses (Barnsteir et al., 2010). While this paper discussed valuable strategies to engage nurses in the use of research, the discussion is limited as effectiveness cannot be concluded and other organizations may not have the resources to implement such an initiative.

Findings of Brown and colleagues' (2009) cross-sectional study further added that a learning environment was the number one facilitator to promoting evidence-based practice among nurses. In an environment where learning and education were valued, nurses expressed an increased desire to enhance their knowledge and skills related to the use of research (Brown et al., 2009). Other authors also discussed education as a facilitator to supporting the use of research among nursing professionals (Chau et al., 2008; Hauck et al., 2013; Karkos \& Peter, 2006; Melnyk et al., 2012; Sandström et al., 2011; Smith \& Donze, 2010; Squires et al., 2012).

When exploring organizational supports for evidence-informed decision-making, the results of Ellen and colleagues' (2013) qualitative study also revealed that participants believed education and training programs facilitated the use of research among professionals. Results revealed that education and training was critical to building capacity to use research at the individual level. The concept of individual capacity is also important to foster because without the appropriate knowledge, skills and willingness to engage in RU, even organizational support may not be enough to promote RU. A literature review by Sandström and colleagues (2011) further suggested that leaders were in an optimal position to address the concerns of nurses related to research use, encourage nurses to engage in RU and also provide them with educational opportunities to enhance their knowledge and skills related to RU.

\section{Literature with Senior Nursing Leaders}

Although the perspective of the staff nurse was a focus of the literature review, the 
importance that nursing leaders hold in facilitating RU was also discussed in many sources. From the 23 empirical articles included in this literature review, only six articles incorporated nursing leaders exclusively or in combinations with other healthcare professionals, and only one of these six was a quantitative study. Hauck and colleagues' (2013) descriptive study assessed the impact of seven leadership facilitation strategies on participants' beliefs of the importance and frequency of evidence use and organizational readiness for evidence-based practice. The participants in this study included nurses with varying levels of time at the bedside and nursing directors or leaders. Some of the strategies implemented included: forums to discuss evidencebased practice, unit level champions to role-model use, promoting an evidence-based practice culture, dissemination of examples of evidence-based practice and creating a rewards system.

When comparing impact before and after the facilitation strategies, scores for beliefs and organizational readiness to engage in the use of research improved significantly and further analysis revealed front line care nurses improved the most (Hauck et al., 2013). After implementing the strategies, the authors also highlighted that leaders were critical for infrastructure development in the following areas: integrating the outcomes associated with evidence use in the strategic plan of the organization, supporting mentors and advocating for resources to educate and train nurses and disseminating information regarding the outcomes of using research (Hauck et al., 2013). It is important to note that the number of leaders that participated in the Hauck and colleagues' (2013) study was small, 10\% ( $\mathrm{n}=43)$ at baseline and $7 \%(\mathrm{n}=32)$ final sample.

A case study by Gerrish and colleagues (2012) aimed to identify the factors that influenced the integration of evidence-based practice among front-line nurses. The study revealed individual attributes of nursing leaders, relationships between leaders and front-line 
nurses, the leader's role and context impact the facilitation of evidence-based practice (Gerrish et al., 2012). The case study highlighted the need for leaders to be knowledgeable about RU, establish credibility, and have a trusting and respectful relationship with nurses. It is important to note the sample of this case study was 23 advanced practice nurses and while their roles differ from senior nursing leaders, the factors mentioned above are critical for professionals in any leadership position to have and they directly inform the present study. More specifically, the factors discussed above may be important to consider when exploring senior nursing leaders roles in supporting RU and how this translates into having adequate organizational capacity to support RU. Another case study of 51 nursing managers investigated their roles in implementing evidence-based practice (Wilkinson et al., 2011). Wilkinson and colleagues' (2011) findings highlighted that the role of managers is not well articulated, hindered by competing demands of managers and largely influenced by contextual factors. Nursing managers highlighted that managerial and administrative duty often over-shadowed the time that could be dedicated to clinical practice and implementing evidence-based practice.

While the studies above were focused on the role of leaders, their relationship with frontline nurses and the barriers they face, Ellen and colleagues' (2013) qualitative study was most closely aligned with the present study. This study explored the supports that healthcare organizations had and perceived to be important for facilitating evidence-informed decisionmaking. Ellen and colleagues (2013) conducted 57 interviews with individuals in three different types of positions (senior management, library manager, and knowledge broker) from regional health authorities, hospitals or primary care practices in Ontario and Quebec. The findings suggested that having a role that actively promoted RU in the organization, linked researchers and opinion leaders, created technical infrastructure and promoted education and training 
facilitated evidence-informed decision-making. This study also stressed the importance of a climate that is receptive to $\mathrm{RU}$ as this promotes the development of individual capacity to engage in RU and also builds the foundation for other initiatives that support the use of research.

In a literature review, Sandström and colleagues (2011) explored the influence of leadership on the process of implementing evidence-based practice. Their findings were divided into three areas: characteristic of the leader, organization and culture. Characteristics of the leader that facilitated RU included; being knowledgeable and committed to research use, visible, accessible, motivating, acknowledging and appreciating nurse efforts, creating opportunities for education and training and providing feedback regarding nurses' use of research (Sandström et al., 2011). The provision of administrative support, policy revisions, allocation of resources, strategic goals and access to library and computer resources were highlighted as characteristics of the organization. Multiple studies in the review also stressed the importance of education in promoting research use, having a quality monitoring system and providing access to a research expert or developing and internal research committee (Sandström et al., 2011). The review also discussed the importance of leaders in creating a culture where RU was valued and incorporated into performance appraisals (Sandström et al., 2011).

\section{Summary of the Literature Review}

A review of the literature suggests that engaging nurses in the use of research is a complex undertaking that requires the presence of many components. Sources highlighted the importance of culture and values that illustrate an organizations' support for the use of research among professionals. The lack of time, resources, access to mentors and rewards were key barriers that impact the use of research in practice. Another strong theme in the literature was the importance of education and training in facilitating RU. The literature review was central to 
establishing an understanding of organizational capacity. More specifically, this review was key in revealing factors, such as a supportive organizational culture, education and training for nurses, support from leaders, that are indicative of organizational capacity and how or if these factors contribute to the use of research. The limitations of the literature included extensive focus on the individual attributes of nurses that impact RU, limited focus on the components of organizational capacity and limited empirical research involving senior nursing leaders. These limitations need to be addressed in order to further improve understanding of organizational capacity and RU from a Canadian context.

\section{Gaps in the Literature}

After a review of the literature, it was apparent that there were gaps that should be addressed by future research. There was limited research exploring organizational capacity in relation to RU. Moreover, a majority of the literature reviewed consisted of samples of front-line staff nurses, signifying an under-representation of the views of senior nursing leaders. In addition, the scarcity of literature from Canada is problematic. Of the literature reviewed, only four studies were conducted in a Canadian context (Ellen et al., 2013; Estabrooks et al., 2003; Scott \& Pollock, 2008; Squires et al., 2012), of which two were from Ontario (Ellen et al., 2013; Squires et al., 2012). A majority of the research reviewed was from areas such as the United States, Australia, Austria, China, United Kingdom, Turkey, Finland and Sweden, where the health-care system differs from Canada and this has implications for the generalizability of results. And lastly, the lack of quantitative research exploring components in the organization with senior nursing leaders reveals the need for more exploration in this area. While other forms of research are valuable, it is important to conduct quantitative research to being to empirically validate and substantiate previous qualitative findings. 


\section{Significance of this Study}

The importance of this study was in addressing the gaps in the literature in four main areas. There was a need for research that: 1) focused on organizational capacity in relation to support for RU, 2) is quantitative, 3) with senior nursing leaders, 4) from Ontario.

\section{Need for research focused on organizational capacity in relation to research}

utilization. While there were meaningful findings regarding the organization in this review, it was not the focus among a majority of sources. Through the literature review, it was revealed that the focus of many sources was to explore the individual attributes of nurses that impact RU. There has been no study, to the knowledge of the researcher, which explored the extent to which components of organizational capacity that support RU are present in organizations. The current study addressed the lack of research on organizational capacity in relation to RU.

Need for quantitative research. While qualitative research is important, it is also critical to further validate these descriptions empirically. Furthermore, descriptive quantitative research is important as it can establish a baseline understanding in a particular area that can then be used to inform other types of quantitative research that focuses on determining the presence of relationships or infer cause and effect between variables (Burns \& Grove, 2009). The current study would generate preliminary findings regarding the presence of components of organizational capacity that support RU.

Need for research with senior nursing leaders. A majority of the literature drew from samples of front-line staff nurses, a group that may not have a higher level of understanding regarding components of the organization that impact RU. The role of senior nursing leaders was revealed as critical when discussing support for RU at an organizational level. As senior nursing leaders have the power and authority to examine and initiate practice change, their input 
regarding how an organization does or does not support RU is critical. Many of the organizational components that were explored in this present study required input from a professional with increased knowledge about the organization and the supports that are in place to facilitate RU. The review of the literature highlighted the need for more research that directly involved senior nursing leaders, as their roles are critical to supporting RU. Therefore, the importance of this study was its involvement of senior nursing leaders when exploring the components of organizational capacity that support RU.

Need for research from Ontario. Because variability in an organization's ability to support RU is largely influenced by context, the need for research that is conducted in the setting to which it is meant to be applied is imperative. Given that the health-care system, practice of nurses, availability of resources and priorities are influenced by context, more research that is representative of a Canadian context is needed. Therefore, it is necessary that research, which accounts for the health-care system structure in Ontario be conducted, and the importance of this study lies in addressing this gap.

This study was among one of the first to address the factors discussed in this review from a Canadian perspective. Furthermore, with a lack of research in the areas mentioned above, this study provides a preliminary understanding of the components of organizational capacity that are present and support RU in organizations in Ontario. Ultimately, this research may be used to create a strategic plan that addresses ways to improve support for RU among senior nursing leaders and nurses. 



\section{Chapter 3: Theoretical Framework}

The purpose of this study was to begin to address the gaps identified through a review of the literature. More specifically, in order to explore the extent to which senior nursing leaders report the presence of components of organizational capacity that support research use, the SUPPORT series was used to guide the study framework. While the SUPPORT series and tool used the term evidence-informed health policymaking (EIHP), which stems from evidenceInformed Decision-Making (EIDM) (Lavis et al., 2009), this study used the term research utilization (RU). The SUPPORT series was used as a guiding perspective as it is the only source that provides a measure of organizational capacity. Furthermore, the subscales and items of the tool highlight components of organizational capacity, which are important to explore when analyzing support for the use of research.

\section{Use of the term Research Utilization}

The term RU was used in this study because it solely focuses on the use of research and it broadly captures the use of research in a manner that can be applicable to the wider nursing profession. RU was defined in this study as: "the use of research findings in any or all aspects of one's work as a registered nurse" (Estabrooks, 1998, p.19). When considering the option to use the term evidence-informed health policymaking (EIHP), it is important to note that this term focuses on the process of using research in policymaking and also specifies how research can be used. The term RU was used because of its broad description of how research is to be used in a manner that it is not limited to a specific group of nurses or to a particular area.

Furthermore, because the target population of this study was senior nursing leaders, use of the term EIHP would not be appropriate as the roles and responsibilities between the population in this study and policymakers may differ. In this sense, the term RU would resonate 
more with leaders as opposed to the term EIHP. In addition, the evidence in EIHP signifies that the term is broad because it includes others forms of knowledge besides research, but the present study was explicitly interested in exploring the use of research as opposed to other sources of evidence. RU, as suggested by the name is solely concerned with the use of research.

While this study used the term RU, it is important to understand the theoretical underpinning and development of evidence-informed health policymaking (EIHP), which is rooted in evidence-informed decision-making (EIDM), as it is a critical part of the series and the tool that was used in this study. EIDM is defined as "the process of distilling and disseminating the best available evidence from research, practice and experience and using that evidence to inform and improve public health policy and practice" (Ciliska, 2012). This term came about as a result of resistance to the evidence-based medicine movement, which was argued to give preference to randomized control trials and meta-analyses. With the introduction of EIDM, it was anticipated that the term would be more inclusive of others forms of evidence that can be used when making decisions (Ciliska, 2012). EIDM has been further expanded to include terms such as: evidence-informed practice, evidence-informed public health, evidence-informed policy making and evidence-informed health policymaking. Regardless of the term used, the underlying premise is the use of multiple sources of evidence, including research evidence, when making decisions (Ciliska, 2012).

\section{Use of the SUPPORT Series as a Guiding Perspective}

Despite some of the differences discussed above, the SUPPORT series was used as a guiding perspective in this study for several reasons. Although the target audience of the SUPPORT series and EIHP are policymakers, the team discusses the need for research use among those who have the power and authority to make decisions (Lavis et al., 2009). Because 
the current study targeted senior nursing leaders, it can be argued that the series is also applicable to them and this study because this group of professionals also has the power and authority to make decisions. Furthermore, the focus of EIHP and the tool that assesses organizational capacity is on the process of using research, which is a component of both EIHP and RU.

Another reason that the SUPPORT series was used in this study was the lack of literature exploring organizational capacity in relation to the use of research and the absence of tools measuring organizational capacity. The series and tool were used as they provide a good measure of organizational capacity and its components. The next section discusses EIHP in relation to assessing organizational capacity to support RU in this study.

\section{Evidence-Informed Health Policymaking}

Essentially, evidence-informed decision-making (EIDM) is a model that provides users with a systematic process that can be used to guide decision-making. There are seven steps in this model including: asking a clear question or problem, acquiring the best evidence, appraising the evidence, integrating the evidence, adapting it to a local context, applying the evidence and analyzing the effectiveness of the application (Ciliska, 2012). Evidence-Informed Health Policymaking (EIHP) is another term that comes from EIDM and it is an approach to decision making in policy that strives to ensure the process is informed by research evidence (Oxman, Lavis, Lewin \& Fretheim, 2009). Evidence-Informed Health Policymaking (EIHP), like EIDM, also follows a systematic process to ensure evidence is used for decision-making. While EIDM is focused on the process of using evidence, EIHP also considers the steps that are required before research can be used. Namely, EIHP also acknowledges the background knowledge and support that is required from the organization in order for the process of using research. 


\section{Steps of Evidence-Informed Health Policymaking}

The SUPporting POlicy relevant Reviews and Trials (SUPPORT) series was led by a group of researchers who have outlined the steps required when using research in policy related decisions (Oxman et al., 2009). The SUPPORT series was developed in order to support individuals, mainly policymakers, who are responsible for making decisions about health policy and programs (Lavis et al., 2009). The series is divided into four main areas, which are further divided into the steps of EIHP: 1) supporting evidence-informed policymaking (defining EIHP, improving how your organization supports EIHP and setting priorities for EIHP), 2) identifying the need for research evidence (using research evidence to: clarify a problem, find options to address the problem and address how the options will be implemented), 3) finding and assessing the evidence (related to finding systematic reviews, deciding how much confidence to place in a review, assessing applicability and other types of evidence), 4) going from research evidence to decisions (engaging stakeholders and using research in decisions) (Lavis et al., 2009).

Improving how an organization supports EIHP. While the EIHP series follows the process and model of EIDM, of particular importance in this study was the first area surrounding supporting evidence-informed policymaking, and more specifically the sub-step about improving organizational support for EIHP. As outlined above, in order to support EIHP professionals need to know what EIHP is, organizations need to improve support for EIHP and also set priorities for EIHP. Oxman and colleagues (2009) posit that in order to improve support for EIHP, lack of organizational arrangements and procedures for setting priorities, which are common barriers, must be addressed. Furthermore, in order to address these barriers the group of researchers suggests that organizations need to assess their organizational capacity to support research evidence use and EIHP (Oxman et al., 2009). 
Assessing organizational capacity to use research evidence. In order for an organization to improve research use and EIHP, it is important to evaluate the supports that are already in place and identify where there is a need for improvement. A number of validated instruments measuring the competence of professionals when using research in practice exist, however tools assessing the organization, namely capacity, are limited (Oxman et al., 2009). The authors present a tool for organizations to assess their capacity to support research use as part of the SUPPORT series. In addition, this tool also generates discussion surrounding an organization's strengths, weaknesses, priorities and areas for improvement. The Organizational Capacity Instrument was used to operationalize organizational capacity in this study. Details about its development are discussed in the next chapter. The remainder of this chapter provides conceptual and operational definitions for the variables of interest in this study (organizational capacity, facilitators and barriers) and concludes with the research questions that were used to guide this study.

\section{Organizational Capacity}

Conceptual definition. Although relatively unexplored in nursing and healthcare, organizational capacity has some established definitions. While there are differences in how various sources have conceptualized organizational capacity, similarities are also noted. In analyzing the indicators of organizational capacity, the most commonly mentioned indicators, among five sources are: resources $(n=4)$, routines/processes or procedures $(n=4)$, organizational culture and values ( $\mathrm{n}=3)$ and infrastructure $(\mathrm{n}=2)$ (Cohen et al., 2013; Hamel \& Schrecker, 2011; Jokelainen et al., 2011; Meyer et al., 2012; Shea et al., 2014).

Although there was commonality among the sources, some differences were also noted. For example, while resources were mentioned four times, some sources broadly mentioned 
resources (Cohen et al., 2013; Jokelainen et al., 2011) and others specified the types of resources required (Meyer et al., 2012; Shea et al., 2014). Cohen and colleagues (2013) were the only source to discuss: knowledge, attitude and skill of professionals, a will or commitment to act, ethics and leadership. In analyzing some of the other differences, the only source that mentioned the size of an organization, decision-making structure and inter-organizational relations was from Meyer and colleagues (2012), who also had the most comprehensive list of indicators. It was interesting to note that none of the sources discussed monitoring and evaluation or a similar component, yet organizational capacity should involve the use of continuous monitoring to some extent. This is important as a change in circumstances may have an influence on organizational capacity, and it becomes important to revaluate the concept at that time.

The outcomes of organizational capacity varied greatly and were dependent on the context in which the term was being discussed. Some outcomes mentioned by the sources included: reduction of health inequities (Cohen et al., 2013), effective mentorship for nursing students during placements (Jokelainen et al., 2011), predictor of organizational processes and performance of employees (Meyer et al., 2012), and the introduction, implementation and sustainability of a new practice (i.e. use of electronic health records) (Shea et al., 2014).

There was only one other source that discussed organizational capacity in relation to the use of research from a healthcare, policy-making lens (Hamel \& Schrecker, 2011). Using case study methodology, Hamel and Schrecker (2011, p.33) defined capacity to acquire research as "the organizational processes and resources to locate, obtain, and assess the quality and relevance of research findings it needs; access to sources of information; research partnership; and staff with research methods skills". Interestingly, both Hamel and Schrecker (2011) and Oxman and colleagues (2009) discussed the need for a process to obtain research, the importance 
of resources and access in this step and skilled people to facilitate this process. Furthermore, both sources acknowledged the need to interpret and transform research before use. In addition, just as Oxman and colleagues (2009) described the use of research as a process, Hamel and Schrecker (2011) also present it as a comparable multi-step process.

While there was some variability in the definitions, indicators and outcomes and of organizational capacity among sources, Oxman and colleagues' (2009) perspective was chosen to guide this study. To begin with, given the target population was senior nursing leaders in healthcare settings an instrument that discussed organizational capacity in relation to the use of research from a healthcare perspective was needed. Furthermore, this was the only source that developed a tool to assess organizational capacity to support the use of research, which was aligned with the purpose of this study.

While Oxman and colleagues (2009) do not offer a formal definition of organizational capacity, their discussion surrounding the term was used when developing a definition for this study. In this study, organizational capacity was defined as an assessment of the extent to which organizations have the necessary conditions, resources and processes to be able to support the use of research evidence (Oxman et al., 2009).

Operational definition. Organizational capacity was operationalized using the Organizational Capacity Instrument (Oxman et al., 2009). Each subscale on the tool corresponds to a component of organizational capacity, whose presence is theorized to support research use among health professionals. The seven components include: organizational culture and values; setting priorities to obtain research; obtaining research; critically appraising research; using research in practice; monitoring and evaluating impact of use and providing professional development (Oxman et al., 2009). The extent to which components are present within an 
organization are believed to reflect an organizations' capacity to support research use.

Organizational culture and values. Some of the items which measure organizational culture and values on the instrument include: mission and key organizational documents supporting evidence-informed decisions, support from leaders, participation in networks and regular meetings to discuss use of research in relation to decisions (Oxman et al., 2009).

Setting priorities to obtain research. An organization that does a good job of setting priorities for obtaining research has criteria for setting priorities, an appropriate mix of people to make decisions about priorities and an appropriate process for setting priorities (Oxman et al., 2009). Once these items are in place, the priorities for obtaining research within the organization can also be established.

Obtaining research. Obtaining research involves accessibility to research. Some of the elements required to enhance accessibility to research include having skilled staff to search and retrieve research, having enough time, incentive and resources, arrangements with external experts and access to databases (Oxman et al., 2009).

Assessing the quality and applicability of research and interpreting the results. Once professionals have access to research, assessing the quality and applicability of research is also critical. The research also needs to be interpreted for use. This component involves analyzing research beyond its methodological characteristics to include an assessment of the context to which research is to be applied. Items such as access to skilled staff, provision of time, incentive and resources and arrangements with external experts are used to quantify this component (Oxman et al., 2009).

Using research. While the Organizational Capacity Instrument did not measure the act of research use, it evaluated organizational support for using research evidence. The subscale aims 
to explore the presence of resources that are needed when professionals use research in practice.

Some of the items on this subscale include provision of time, expertise and incentive, stakeholder involvement, knowing how and when research can be used and transparency of the how and what evidence was used in decisions (Oxman et al., 2009).

Monitoring and evaluation. The discussion of monitoring and evaluation is limited to policies and programmes on the Organizational Capacity instrument from Oxman and colleagues (2009). Although the series does not specify the types of policies and programmes to be evaluated, one might infer the relation to policies and programmes on evidence-informed health policymaking. Items such as considering the need for monitoring and evaluation, having access to external experts or staff with expertise, provision of incentives and resources and involvement of stakeholders were measured on the subscale (Oxman et al., 2009).

Supporting continuing professional development. Oxman and colleagues (2009) indicate that continuing professional development should address important topics and be evidence-based. The provision of professional development might involve educating professionals about the different components measured on the instrument. For example, organizations may be involved in providing professional development opportunities related to searching for and retrieving research, assessing the quality of research or evaluating the effectiveness of a policy that promotes RU. Organizational support of professional development opportunities was measured with items that explore time for professional development, routines to develop skills in accessing, appraising and applying research, staff priority of engaging in professional development, and the provision of internal and external professional development opportunities (Oxman et al., 2009). 


\section{Facilitators}

Conceptual definition. Facilitators were defined in this study as the factors within an organization that support the use of research. The previous chapter highlighted that the presence of certain factors can contribute to an organizations' capacity to support the use of research. When present, these factors, known as facilitators, assist nurses with RU. Facilitators provide nurses with the necessary resources (time, financial, human and technical), knowledge and skills, and infrastructure (procedures, processes and policies) required to be successful in the use of research. If effective, facilitators may change nurses' attitudes and beliefs about RU, promote their interest in RU, enhance the knowledge and skills required, provide access to the resources required and ideally increase engagement with RU. For example, in the previous chapter organizational culture was discussed as one of the most cited facilitators. When the culture and values of an organization, which are evidenced through supportive mission and vision statements, support from leaders and committed resources, illustrated support for the use of research there was an impact on how nurses viewed RU. These views might in turn impact nurses' attitudes and engagement in RU (Brown et al., 2009; Thiel \& Ghosh, 2008). Therefore, if elements of organizational culture are positive and they assist nurses with engagement in RU, this would exemplify a facilitator of RU.

Operational definition. Senior nursing leaders self-report of the factors that support research use within their organization.

\section{Barriers}

Conceptual definition. Barriers were defined in this study as the factors that hinder the use of research within an organization. The absence of these factors creates circumstances or conditions, which become a hindrance for nurses engaging in RU. Similar to the discussion of 
facilitators, if organizations do not have the necessary resources, or education and training that facilitates the development of required knowledge and skills, or infrastructural supports, this becomes a barrier and impacts how engaged nurses are in RU.

The same factors that can be facilitators to RU can also be barriers if they are not supportive or are absent within an organization. For example, while organizational culture was discussed as a facilitator by some sources reviewed in this study, another source reported that it was a barrier (Melnyk et al., 2012). This difference is related to how supportive or unsupportive a factor is of RU. While some organizations may have resources, provide knowledge and skills and have the infrastructure that supports RU, another organization may not and these same factors would be classified as barriers in the other organization. Some barriers might include lack of access to resources, inadequate knowledge and skills to engage in RU, negative attitudes and beliefs about RU, lack of interest in RU and decreased engagement with RU.

Operational definition. Senior nursing leaders self-report of the factors that hinder the use of research within their organization.

\section{Research Questions}

The research questions that informed the study were:

1. To what extent do senior nursing leaders report the presence of the following components of organizational capacity, which support RU, within organizations:
a. Organizational culture and values
b. Setting priorities for obtaining research
c. Obtaining research evidence for use
d. Assessing quality and applicability of research evidence and interpreting results
e. Use of research evidence 
f. Monitoring and evaluating existing policies and programs related to research use

g. Supporting continuing professional development to assist with use of research evidence in practice

2. What do nurse leaders describe as facilitators and barriers to research utilization within their respective organizations? 


\section{Chapter 4: Methods and Procedures}

\section{Design}

A descriptive, quantitative design was used to examine the presence of components of organizational capacity that support RU in various settings in Ontario. This research design is most appropriate when little is known about a topic, thus warranting a need to develop greater understanding (Burns \& Grove, 2009). Although there is research on organizational factors related to research use, there is limited exploration of organizational capacity and the organizational facilitators and barriers to research use in Ontario. This study involved the use of a questionnaire and two open-ended questions to elicit information from senior nursing leaders about organizational capacity and the facilitators and barriers to RU.

\section{Sample}

Target and accessible population. The target population included registered nurses in senior leadership positions within various healthcare settings in Ontario. As it is difficult to identify and access all leaders in Ontario because the exact number of leaders is unknown and they work in a variety of geographically dispersed areas, there was a need to narrow the target population to a more focused and accessible population. The accessible population for this study included senior nurse leaders who were members of the Nursing Leadership Network of Ontario (NLN.ON) interest group offered by the Registered Nurses of Association (RNAO), including those who are members of the groups' Facebook and LinkedIn page. In addition, the accessible population included nursing leaders on the Ontario Ministry of Finance (2014) public salary disclosure list.

Inclusion and exclusion criteria. The inclusion criteria for this study were (a) registered nurses in (b) the following positions: Chief Nursing Officers, Directors, Managers, Vice 
Presidents and Presidents. Professionals holding these various positions were included in this study as they: understand the organization, have the power to influence the capacity of the organization, have the capability to make decisions, and have professional relationships with staff nurses in direct and indirect capacities (Rycroft-Malone et al., 2004). There were no restrictions placed on the inclusion of participants from various settings as this study was exploratory in nature and this promoted increased representativeness of the population. Therefore participants from acute care and community settings were eligible to participate in this study.

Exclusion criteria for this study were registered nurses who were working as (a) frontline staff, (b) charge nurses, (c) resource nurses, (d) educators, (e) advanced practice nurses, (f) nurse practitioners and (g) nurses with temporary leadership positions. For the purpose of this study, a temporary position referred to one in which an individual was replacing another for an indefinite or definite period of time; otherwise meaning the position was not permanent. The professionals listed above were excluded, as they may not have the power and authority to make changes and decisions in the same capacity as leaders in senior positions. In addition, individuals among the excluded list of positions may not possess the higher-level understanding of the organization, be able to assess or influence organizational capacity to support research use or realize the impact organizational capacity has on practice. Furthermore, individuals in these roles may not have reporting relationships with executive and/or corporate leaders or have the power and authority to influence organizational capacity or its' components and ultimately encourage RU among nurses (Cummings, Estabrooks, Midodzi, Wallin \& Hayduk, 2007; Matthew-Maich, Ploeg, Jack \& Dobbins, 2010; Salmela et al., 2013).

In order to ensure participants met the inclusion criteria, the researcher placed a reminder on the information and consent page and at the beginning of the online questionnaire that stated: 
"If you currently work as a front-line staff or charge or resource or advanced practice nurse, team leader, educator, nurse practitioner or are in a temporary leadership position, we thank you for your time, but you do not meet the study eligibility criteria. Please do not continue with the study."

Sampling method and recruitment. Several different sampling strategies were used to maximize the sample size in this study and included: (1) the online newsletter, Facebook and LinkedIn page of the NLN.ON, (2) those referred by other leaders and (3) those on the Ontario Ministry of Finance (2014) public salary disclosure list.

As all leaders in Ontario were not accessible to the researcher, a non-probability convenience-sampling method was initially used for this study. The researcher consciously selected to recruit from a group where nursing leaders are easily accessible (the NLN.ON website and their Facebook and LinkedIn page). Using the interest group for the purposes of recruitment was beneficial as it is one of the only consortiums in Ontario which can be used to identify nursing leaders in various organizations and settings. Cullum, Ciliska, Haynes and Mark explain that in convenience sampling, participants are selected by the researcher based on “...ease of access... and, secondarily, for their knowledge of the subject matter" $(2008$, p.208). Furthermore, considering senior nursing leaders work in various settings and organizations which are geographically dispersed and it is difficult to obtain contact information of all individuals in leadership positions, the researcher sought an opportunity to easily access potential participants at one time through the NLN.ON newsletter, Facebook and LinkedIn page.

The second part of Cullum and colleagues (2008) description of convenience sampling is also relevant to this study as senior nursing leaders were recruited because of their in-depth knowledge and expertise regarding the organization, and more specifically the components of 
organizational capacity that are important when considering support for research utilization. Burns and Grove (2009) also highlight the usefulness of this sampling method when exploring areas in which research is not well established. Because convenience sampling is the most cost effective, promotes ease of accessibility and suitable considering the exploratory nature of this study, it was utilized (Burns \& Grove, 2009; Cullum et al., 2008).

A recruitment advertisement in the online NLN.ON newsletter was used as the group does not meet regularly and a shorter version of the advertisement was posted on the group's Facebook and LinkedIn page. The advertisement (please refer to Appendix C) included the purpose of the study, what participation in the study consisted of, the time commitment required, contact information of the researcher and instructions for accessing the online survey. Senior nursing leaders were instructed to contact the researcher regarding any questions and were also provided with a link to access the survey online. Once on the survey webpage, leaders were instructed to read detailed information about the study and answer a question regarding consent, after which the survey was initiated. If leaders provided a name or contact information of other potential participants, the researcher contacted these individuals. This alternative strategy was classified as snowball sampling (Burns \& Grove, 2009).

The final recruitment strategy involved the researcher accessing a publically available salary disclosure list for Hospitals and Boards of Public Health from 2013 in order to obtain names of senior nursing leaders throughout Ontario (Ontario Ministry of Finance, 2014). Employing purposive sampling, the list was filtered using the terms 'nursing' and 'nurse'. This strategy was purposive as the list contained all healthcare professionals who made over a certain salary cut-off, but the researcher was only interested in senior nursing leaders. Upon reducing the list to senior nursing leaders, contact information for the individuals was obtained using 
organization websites and publically available contact information. If needed, hospital administrators were contacted to obtain email addresses of the leaders. The leaders were then emailed the recruitment advertisement and directed to the research study's online webpage.

For participants recruited through the salary disclosure list, multiple follow-ups were used to enhance participation. Applying the Dillman, Smyth and Christian (2009) method for Internet surveys, follow-up from initial contact was conducted twice, with a two-week gap in between each follow-up. Dillman et al. (2009) recommend two to three follow-up attempts for Internet surveys and this guide was followed in this study. Participants were recruited using these methods until the minimum desired sample size was met and the survey was closed September 30, 2014 after which access to the survey was not available.

Sample size considerations. Due to the descriptive nature of the study, sample size was not calculated through a power analysis. Because this study was the first to examine organizational capacity and its components from the perspective of senior nursing leaders across healthcare organizations in Ontario, it was treated as a preliminary study. In exploring sample size determination strategies for preliminary and pilot studies, Billingham, Whitehead and Julious (2013) reveal there is a lack of published guidelines for researchers. The authors report on the most cited methods when determining sample size in pilot and feasibility studies. Of the 79 articles in Billingham and colleagues (2013) reviewed, justification for sample sizes ranged from: targeting 0.03 times the sample size planned for the larger study (Stallard, 2012), a general rule of thumb of 30 participants to estimate a parameter (Browne, 1995), a minimum of 12 participants per group (Julious, 2005) and having 50 participants per group (Sim \& Lewis, 2012).

In rationalizing the target sample size for this study, the first method by Stallard (2012) did not apply, as this study did not precede a larger trial, the last two methods by Julious (2005) 
and Sim and Lewis (2012) were also not applicable, as this study was not experimental and therefore did not have a control and experimental group. A review of the literature for response rates of senior nursing leaders also yielded no data. Another guideline that was used to estimate sample size requirements in this study was the recommendation of having five-ten participants for every variable analyzed in a study (Burns \& Grove, 2009). Based on this guide and the three variables of interest in this study (organizational capacity, facilitators and barriers), the researcher aimed for a target sample of 15-30 participants. Browne's (1995) recommendation of having a minimum of 30 participants was also considered and while this study targeted a minimum number of participants, all participants who responded during the data collection period (until September 30, 2014) were included in the analysis. It was not expected that the number of participants recruited would exceed 60 .

\section{Setting}

Data collection occurred in an uncontrolled, naturally occurring setting (Burns \& Grove, 2009). The only option of completing this questionnaire was through the Internet. It was anticipated that structuring the study in this manner provided participants the freedom to choose when and where the study was completed and that this would also increase the response rate. A method to minimize extraneous environmental variables in this study involved providing study participants with detailed instructions for completing the survey. All participants received the same detailed instructions from the researcher in an effort to limit errors caused by confusion. It was anticipated that consistency in the delivery of study information would help limit the number of surveys considered incomplete due to errors in participant responses. 


\section{Ethics}

The researcher obtained approval to conduct this study from the Research Ethics Board at Ryerson University (see Appendix D). The initial recruitment strategy of the NLN.ON newsletter (see Appendix C) also required approval from the group's Director of Education and Research and it was necessary for the researcher to join the group as a member.

Participants had the freedom to choose to participate in this study, free from coercion. Furthermore, those who participated were free to quit the study at any time. Participants were also informed that a decision not to participate in the research study would not harm them in any way. Considering the descriptive nature of this study, it was anticipated that the potential risk of participating in this study would be minimal (Burns \& Grove, 2009). Participants were also informed of their right to skip question(s) or stop their participation at any time, should they have felt uncomfortable. Although there were no direct benefits expected for the participants, the researcher anticipated findings from this study would contribute to knowledge generation about RU. Monetary benefits were not offered in this study.

All potential participants were able to access the URL link independently or they were directed to the webpage of the study if they contacted the researcher directly. Before participants proceeded to the online questionnaire, the first page of the URL link contained detailed information about the study and consent process (please refer to Appendix E). After reading this first page, participants were prompted with the statement: Answering yes to the question below will indicate that you have read and agree with the information on this page. Do you consent to participate in this survey? Consent for participating in the study was implied when participants answered yes to this question. An implied consent process was appropriate because the study had minimal risk associated with participation (Burns \& Grove, 2009). Once a participant consented 
to participate, their responses were included regardless of if they: completed the survey and submitted, skipped question(s) and submitted or if they quit/stopped the study without submitting (an incomplete survey). This information was clearly outlined in the study information provided to potential participants.

Another element in this study was the anonymity of study participants. This was facilitated through the anonymity feature of the website used to build the study questionnaire. The researcher was not able to link completed questionnaires with participants in this manner. Informing participants of this process was crucial as it was anticipated this would allow participants to answer freely when completing the questionnaires. Although this method potentially minimized social desirability bias, which is the tendency to present responses that are considered ideal, a limitation posed is the inability to withdraw responses once a participant submits a completed survey (Loiselle et al., 2007). In order to protect participants' rights, this information was included in the letter of information and consent so that participants were aware that they could not withdraw their responses once submitted. Another limitation of anonymity was the inability for the researcher to follow-up with participants in case clarification was needed. Regardless of these limitations, the researcher prioritized anonymity of participants to facilitate honest responses from those participating in the study.

Confidentiality was maintained by protecting data collected during and after the completion of the study. All electronic data were kept on a password-protected, encrypted external device and the files were only accessible to the researcher. Demographic data of the participants, including their work setting, were collected using codes and categories to minimize the risk of being able to identify participants. Participants were also asked not to include any 
identifying information on their responses to the study questionnaires. The researcher will destroy the data one year after completion of the study.

\section{Measurement}

Two instruments were used with senior nursing leaders to collect pertinent data (a) Demographic Data Questionnaire and the (b) Organizational Capacity to Support the Use of Research Evidence to Inform Decisions Instrument (Oxman et al., 2009).

Demographic Data Questionnaire. Demographic information was collected using a researcher developed Demographic Data Questionnaire (please refer to Appendix F) in order to describe the sample. Participants were asked to complete ten questions about: (1) sex, (2) age, (3) number of years as a registered nurse, (4) level of education (nursing), (5) level of education (non-nursing), (6) number of years in a leadership position (including past positions), (7) current position, (8) number of years in current position, (9) practice setting and (10) organization size.

\section{Organizational Capacity to Support the Use of Research Evidence to Inform}

Decisions Instrument. The main instrument used to operationalize organizational capacity was the Organizational Capacity Instrument by Oxman and colleagues (2009). This instrument can be used as an assessment of an organizations' capacity to use research evidence when making decisions. More specifically, it contains subscales that measure components, which are thought to increase an organizations' capacity to use research. This instrument was developed by building on the 'Is Research Working For You' tool by the Canadian Health Services Research Foundation (CHSRF) and the 'Assessing country-level efforts to link research to action' framework by Lavis, Lomas, Hamid and Sewankambo (2006) (Oxman et al., 2009). Addressing deficiencies in the CHSRF tool and the Lavis and colleagues (2006) framework, Oxman and 
colleagues (2009) developed this instrument to assess and improve the organizational component of using research in practice.

The Organizational Capacity Instrument was relevant for this research study as it explores an organization's capacity to use research evidence by assessing the degree to which components of organizational capacity that support RU are present. The Organizational Capacity Instrument is a scorecard-based tool that was developed following a survey of 176 organizations and more detailed telephone interviews with 25 organizations. Drawing on the lessons learned from surveys and interviews, Oxman and colleagues (2009) suggested five questions are important when considering how to support research use among organizations. This particular instrument addresses the first of five questions and is directly aligned with the purpose of this research study: "What is the capacity of your organization to use research evidence to inform decision-making?" (Oxman et al., 2009, p. 3).

The instrument consists of a total of 36 items divided among seven subscales: (a) organizational culture and values that support the use of research, (b) setting priorities for obtaining research evidence to inform decisions, (c) obtaining research evidence to inform decisions, (d) assessing the quality and applicability of research and interpreting the results to inform decisions, (e) using research evidence to inform recommendations and decisions, (f) monitoring and evaluating policies and programmes and (g) supporting continuing professional development (Oxman et al., 2009). Subscales are comprised of 4 to 6 items and are scored on a 6-point Likert scale with possible scores ranging from 0 to 5 where 0 represents don't know, 3 represents neither agree nor disagree and 5 represents strongly agree. The stem of each question that forms a subscale is: "Does your organization..." and each item explores a specific subcomponent of each subscale (Oxman et al., 2009). Each item is scored individually, as part of the 
subscale and contributes to an overall score. The possible range of scores for each subscale varies from 0 to 20 to 30 and the total overall score ranges from 0 to 180 (Catallo \& Sidani, 2014; Oxman et al., 2009).

The original instrument also contains three open-ended questions at the end of each subscale to gather more information about the participants' organization and discuss additional strengths and weaknesses. Because these questions were not required to answer the research questions for this study, they were removed from the tool. Removal of the open-ended questions should not impact validity of the tool, as answers to the questions are not accounted for in statistical testing. In order to give participants the option of discussing additional facilitators and barriers to RU, the questions were replaced by two researcher developed open-ended questions. Using this approach was thought to prompt the views' and opinions' of leaders. Participants were asked to respond to the following at the end of the instrument: Please identify any other facilitators that support research use and please identify any other barriers that inhibit research use. Face validity testing was undertaken to determine the appropriateness of the additional questions and supported by consulting with the researchers' thesis committee members.

The Organizational Capacity Instrument evolved through multiple workshops with different groups aimed at addressing content validity (Oxman et al., 2009). Psychometric testing of the instrument is limited as it is fairly new. Using a telephone survey of 57 Canadian health policymakers, preliminary testing of reliability and validity of this instrument has been completed (Catallo \& Sidani, 2014). The authors calculated Cronbach's $\alpha$ coefficients for the seven subscales and item-to-total correlations of this instrument to determine reliability.

Item-to-total. The item-to-total correlations ranged from 0.16 to 0.74 (Catallo \& Sidani, 2014, p.4). The authors used the guideline of $>.30$ as acceptable for the item-to-total correlations. 
Only two items were below the accepted 30 criterion and authors reported this might be due to low variability in scores (Catallo \& Sidani, 2014). These two items were left in the instrument as their removal may have impacted the number of items used to operationalize the respective subscales and consequently compromise quality of the instrument. Overall, the item-to-total correlations were reported as positive and moderate associations.

Within subscales. Internal consistency reliability scores within each subscale were reported as being of moderate to high magnitude. The Cronbach's $\alpha$ coefficient scores ranged from 0.67 to 0.91. Catallo and Sidani (2014) reported the Cronbach's $\alpha$ coefficient was 0.81 for organizational culture and values; 0.91 for setting priorities for obtaining research to inform decisions; 0.67 for obtaining research evidence; 0.82 for critically appraising research evidence; 0.78 for using research evidence; 0.91 for monitoring and evaluating the impacts of decisions and 0.76 for professional development. As this is a newly developed instrument, most coefficient scores were within an acceptable range of > 0.70 (Burns \& Grove, 2009; Catallo \& Sidani, 2014). The authors reported three items had an inter-item correlation above 0.80 indicating redundancy. In response, Catallo and Sidani (2014) calculated Cronbach's $\alpha$ coefficients with and without these items and determined a slight decrease in the $\alpha$ coefficient indicating need for further psychometric testing before the decision to exclude these items could be made.

Discriminant validity. Group comparison was used to provide evidence of discriminant validity. The mean subscale scores were compared for policy analysts and senior managers using the independent sample $t$ test and effect size (Catallo \& Sidani, 2014). The effect size, measured by Cohen's d, ranged from - -10 to .16 , indicating the amount of difference in subscales, between the two groups, was small. Although discriminant validity aims to measure differences, preliminary psychometric analysis revealed the instrument did not discriminate responses among 
the sample of policy analysts and managers. As the researchers expected to see a difference between the two groups, they discussed the limitations of a small sample in their study and indicated the need for further psychometric testing using larger sample sizes. Overall, Catallo and Sidani (2014) report this instrument can be useful when evaluating an organization's capacity to use research evidence but they suggest the need for further psychometric testing.

Although Catallo and Sidani (2014) recommend further psychometric testing, the use of the Organizational Capacity Instrument was very important for this research study. The fit between the conceptualization and operationalization of organizational capacity was very strong with the use of this instrument. The conceptualization of organizational capacity was informed by Oxman and colleagues' (2009) series and it was operationalized was through the selfassessment tool for organizations. In addition to enhancing construct validity through a clear conceptualization and operationalization, use of the Organizational Capacity Instrument was warranted, as this was the only instrument, to the knowledge of the researcher, which was suitable for measuring organizational capacity. Because the aim of this study was to gain an understanding of the presence of components of organizational capacity that support RU, it was very important to choose an instrument that was comprehensive in its inclusion of organizational factors, hence another reason to utilize the Organizational Capacity Instrument.

\section{Data Collection}

The original Organizational Capacity Instrument is a paper-based tool that is accessible on the Internet. Because the researcher used an online questionnaire, items from the instrument and demographic questionnaire were transferred online using Opinio 6.0- an online survey building, publishing and maintaining software (ObjectPlanet Inc.). This software allows researchers to create online surveys, disseminate them to participants and export data to 
programs such as Microsoft Excel and IBM SPSS for analysis. All 36 items from the instrument were entered onto the online survey tool without any modifications. The researcher doublechecked accuracy of the online survey by completing a mock survey and two thesis committee members also reviewed the survey before beginning recruitment. The original tool asks users to identify the organization in which they work; however this question was removed to protect the anonymity of participants. Burns and Grove (2009) discuss a reduction in transcribing errors as a benefit of computerized data collection. By reducing the chance of error that may have resulted from manually recording or transcribing data, this method can also increase accuracy and reliability. Although digital data collection may require considerable preparation, this method saved the researcher time during the data collection and analysis phases (Burns \& Grove, 2009).

\section{Data Management}

All data were coded using a codebook for data definitions. All demographic variables and items from the Organizational Capacity Instrument were identified and defined using (a) shortened, abbreviated variable name, (b) descriptive variable name, and (c) the range of possible numeric values (Burns \& Grove, 2009). These data definitions and codes were used during the analysis process. The codebook was transferred to IBM Statistical Package for the Social Sciences (IBM SPSS) Version 22.0 in preparation for analysis.

While the data were collected, the researcher prepared for analysis by checking for missing data and other problems that may have compromised the integrity of study findings. Because the data were being collected on the computer, the researcher ensured the data were backed up and saved in a secure manner, in case of a technical issue. In order to protect and avoid loss of valuable data, this was done through an encrypted, password-protected external device that was accessible only to the researcher. 


\section{Data Analysis}

All the data from the Demographic Data Questionnaire and Organizational Capacity Instrument were imported into IBM SPSS Version 22.0. Data from the open-ended questions were imported into a separate word document. The following will be discussed below: preparing the data for analysis by testing for missing data, testing reliability of the instrument and approach to analysis for describing the sample and addressing the research objectives.

Missing data. Once data were imported into SPSS, they were evaluated for missing values. Following identification of missing values, they were analyzed to determine the extent and pattern of missing data (Burns \& Grove, 2009). This was done to determine an appropriate approach to handling missing data, while keeping in mind the amount of data that were missing (Fox-Wasylyshyn \& El-Masri, 2005). This study followed Cohen and Cohen’s (1983) recommendation that if there is no more than $10 \%$ missing data, the amount is not large and it should be retained for analysis (as cited Fox-Wasylyshyn \& El-Masri, 2005). Next, the pattern of missing data was explored to establish if the cause was the result of a random or systematic error (Fox-Wasylyshyn \& El-Masri, 2005).

During the first stage, the extent of missing data was calculated as a percentage based on the number of items a participant left unanswered out of a possible 36 items on the instrument. In the case that the missing data totalled less than $10 \%$ and was missing at random, the researcher chose to impute data using the case mean substitution technique (Fox-Wasylyshyn \& El-Masri, 2005). This approach was taken in order to retain the sample size of the study. Furthermore, Polit (2010) suggests imputation techniques are appropriate if up to $30 \%$ of data are missing. In order to complete the imputation, the researcher calculated the individual participant's mean with the 
subscale items that had completed data. This mean was then imputed as the value for the missing response(s) and the subscale mean was then calculated based on a complete set of data.

This technique was chosen for several reasons. To begin with, case mean substitution facilitated the use of completed data from the participant and the imputation value was characteristic of the individual and how they are responding to questions. Furthermore, as this instrument had seven subscales, the completed data from the subscale with missing response(s) was used to calculate a mean for substitution. Because each item on the subscale is representative of the component being measured, the mean calculated should be representative of the particular component being measured (Fox-Wasylyshyn \& El-Masri, 2005). Therefore, using this approach to handle missing data is beneficial as it acknowledges and characterizes the participant and the component being measured and retains the sample size of the study.

Summary of missing data. In this study, the extent of missing data was determined based on the number of items missing from the overall 36 items on the instrument. Seven respondents out of 43 had some missing data on the Organizational Capacity Instrument. The missing data in all seven cases did not follow a particular pattern and was therefore treated as missing at random. Of these seven respondents, five cases had a response missing for one item on any given subscale and this was equivalent to $2.78 \%$ missing data on the total Organizational Capacity Instrument. The remaining two cases had a missing response for two items on any given subscale, which was equivalent to $5.56 \%$ missing data on the total instrument. The “organizational culture and values [that] support the use of research evidence to inform decisions" was the only subscale that had no missing values.

Reliability Testing. In order to assess the internal consistency reliability of the Organizational Capacity Instrument, a Cronbach's alpha coefficient for the instrument and each 
of the seven subscales was calculated using IBM SPSS. An alpha coefficient of .70 or greater was considered acceptable since this is a relatively new instrument (Burns \& Grove, 2009).

Statistical Analysis. Descriptive statistics were used to analyze data collected in order to describe the sample and address the research questions.

Sample. Sex, current position and practice setting were measured at a nominal level. Highest level of education completed and organization size were measured at an ordinal level. These variables were reported using frequency and percentage distributions. Age in years, number of years as a nurse, number of years in leadership positions and number of years in current leadership position were measured on a ratio level of measurement and were reported using measures of central tendency (mean), and measures of dispersion (range and standard deviation) (Burns \& Grove, 2009).

Research questions. Oxman and colleagues' (2009) Organizational Capacity Instrument was used to explore the main research question To what extent do nurse leaders report the following components of organizational capacity as present within their respective organizations? The tool has a total possible range of scores from $0-180$, which represents an overall measure of organizational capacity and suggests the presence of a continuum of values. Therefore, data collected with this instrument were treated as interval level. In order to answer sub-questions a) through g), the mean score for each subscale and the total instrument was obtained to explore the distribution of responses among participants. As measures of dispersion, the standard deviation and range of scores were also reported to demonstrate how scores varied among participants (Burns \& Grove, 2009). In order to obtain more information about the items on a subscale, the mean and standard deviation of the individual items was also calculated. 
The second research question related to facilitators and barriers to research utilization was explored using data obtained from two open-ended questions. Answers to the open-ended questions were analyzed based on the principles of content analysis. The purpose of content analysis is the classification of text into categories or themes that are meaningful; a quantitative approach involves reporting the frequency with which a particular unit of analysis is mentioned (Burns \& Grove, 2009; Elo \& Kyngas, 2007; Hsieh \& Shannon, 2005). The unit of analysis being described is dependent on the purpose of the research and can include words, phrases, categories, themes and more (Hsieh \& Shannon, 2005).

In this study, conventional content analysis was used to analyze data from the openended questions (Hsieh \& Shannon, 2005). A characteristic of this type of analysis is the text drives the development of categories as opposed to preconceived categories. The researcher first reviewed the responses regarding the facilitators and barriers to research use separately. During this first review, the researcher made notes of words and phrases that could be classified into potential categories. Similar responses were then grouped together into a broader category based on meaning. A mutually exclusive and exhaustive category list was then developed from these notes so all data could be grouped into a category. Following this step, the researcher recorded the number of times a category was mentioned and reported them using frequency counts.

In order to ensure accuracy during the process, the thesis supervisor and another Masters prepared colleague with qualitative research experience reviewed the categorization scheme. This process, known as peer debriefing, was meant to enhance credibility and rigor of findings (Speziale \& Carpenter, 2007). The researcher provided the reviewers with a coded transcript of the open-ended responses and included examples of the type of responses that were categorized into a particular category. The reviewers examined the text and categorization of responses into a 
particular category to validate the categorization scheme until there was agreement between the reviewers and researcher. In addition to describing the frequency with which categories were mentioned, the results from the open-ended questions were also described narratively, including the use of participant quotes to supplement the discussion where relevant. 



\section{Chapter 5: Results}

In this chapter, the findings related to the extent to which components of organizational capacity, which support research use, are presented. In addition, findings related to the facilitators and barriers to research utilization as reported by senior nursing leaders are presented. The chapter begins with a description of the response rate and demographic characteristics of the sample, followed by results of reliability testing of the Organizational Capacity Instrument. It concludes with the results pertaining to the research questions.

\section{Sample}

Response Rate. Given the nature of the recruitment strategies used, it was only possible to keep track of the number of participants contacted for one strategy. A total of 99 nursing leaders were emailed from the publically available Ontario Ministry of Finance (2014) salary disclosure list. Of the 99 nursing leaders that were emailed, 26 (26\%) declined to participate. Reasons for refusals or non-participation included: busy/refused to provide contact information $(n=12)$, retiring $(n=2)$, position no longer available $(n=4)$, temporary leader $(n=5)$, selfidentification of not fitting inclusion criteria $(n=2)$ and maternity leave $(n=1)$. Due to the anonymous approach of data collection in this study, the researcher could not calculate response rates for any of the other recruitment strategies used in this study.

A total of 55 participants recruited through the various strategies consented to participate in the survey. It was not possible to determine how many participants came from a particular recruitment source. Of the 55 participants who consented, 36 participants (65\%) completed all demographic information and the Organizational Capacity instrument, 7 participants (13\%) completed some/all of the demographic information and partially completed the instrument (i.e. they had missing values), 8 participants (15\%) completed some or all of the demographic 
information but did not complete any questions on the instrument and 4 participants (7\%) did not provide any demographic data or answer any questions on the study instrument. The final sample size for this study $(n=43)$ was comprised of participants from the groups who completed all demographic information and the Organizational Capacity instrument or completed some/all of the demographic information and partially completed the instrument. Upon analysis of demographic information, such as sex, highest nursing education and size of current organization, the participants who did not complete the survey were comparable to those who did. Age, number of years as an RN and number of years in current leadership position were slightly higher among those who did not complete the survey whereas the sample included in this study had more experience as leaders.

Demographic Profile. The majority of nursing leaders were females $(n=36,84 \%)$ with a mean age of 48.74 years $(\mathrm{SD}=9.94)$. On average, leaders had approximately 25 years of experience as a registered nurse and 16 years in a leadership position. Detailed information regarding the demographic variables is presented in Table 1.

Table 1

Age and number of years as a nurse, nursing leader and in current position

\begin{tabular}{|c|c|c|c|c|c|}
\hline \multirow[b]{2}{*}{ Characteristic } & \multirow[b]{2}{*}{$\mathrm{N}$} & \multirow[b]{2}{*}{ Mean } & \multirow[b]{2}{*}{ SD } & \multicolumn{2}{|c|}{ Range } \\
\hline & & & & Minimum & Maximum \\
\hline Age & 43 & 48.7 & 9.9 & 26 & 62 \\
\hline Number of Years as RN & 42 & 25.6 & 11.2 & 2 & 42 \\
\hline Total \# of Years as Leader & 43 & 16.3 & 11.2 & 1 & 55 \\
\hline $\begin{array}{l}\text { \# of Years in Current Leadership } \\
\text { Position }\end{array}$ & 43 & 6.7 & 5.0 & 1 & 19 \\
\hline
\end{tabular}

Note: The Number of Years as an RN had one missing response. 
Furthermore, a considerable number of leaders reported having a Masters $(n=17,40 \%)$ or Bachelors in Nursing $(n=16,37 \%)$. Specific leadership roles among participants in the sample included: Chief Nursing Officers $(n=16,37 \%)$, Managers $(n=8,19 \%)$, Directors or Associate directors of Nursing $(n=7,16 \%)$ and President or Vice-President $(n=4,9 \%)$. A total of eight respondents (19\%) selected the other category for current position and most of the roles entered in the free text box were a combination of more than one role or participants reported a very specific role. More than half of the nursing leaders reported working in acute care hospitals $(\mathrm{n}=$ $29,67 \%)$, followed by long-term care $(n=5,12 \%)$ and community $(n=1,2 \%)$ settings. While two participants (5\%) did not respond to this question, six participants (14\%) described other settings such as: public health $(n=2)$, mental health $(n=1)$, retirement $(n=1)$, nursing association $(n=1)$ and government $(n=1)$. Additional demographic information is presented in Table 2. 
Table 2

Other demographic characteristics of the sample

\begin{tabular}{cll}
\hline Characteristic $(\mathrm{n})^{*}$ & Frequency & Percentage \\
\hline Highest Nursing Education $(\mathrm{n}=43)$ & & \\
- Diploma & 4 & 9.3 \\
- Baccalaureate & 16 & 37.2 \\
- Masters & 17 & 39.5 \\
- PhD & 6 & 14.0
\end{tabular}

Highest Non-Nursing Education $(\mathrm{n}=37)$

- Diploma

- Baccalaureate

10.8

- Masters

8.1

- $\mathrm{PhD}$

24.3

- Not Applicable

2.7

54.1

Current position $(n=43)$

- Chief Nursing Officer

37.2

- Manager

$\begin{array}{ll}16 & 37.2 \\ 8 & 18.6 \\ 8 & 18.6 \\ 7 & 16.3 \\ 4 & 9.3\end{array}$

- Director/Associate Director

18.6

- President/Vice-President

9.3

Current work setting $(\mathrm{n}=41)$

- Acute care hospital

- Other

$29 \quad 67.4$

- $\quad$ Long-term care

$6 \quad 14.0$

- Community organization

$5 \quad 11.6$

$1 \quad 2.3$

Size of current organization $(n=43)$

- 11-20 nursing staff

- 21-30 nursing staff

- $\quad 31-40$ nursing staff

2.3

- 41-50 nursing staff

- $\quad>50$ nursing staff

*Some missing values

\section{Organizational Capacity Instrument Reliability Testing}

Internal consistency reliability for the 36-item Organizational Capacity Instrument was supported by a Cronbach's alpha coefficient of $0.96(n=43)$. Cronbach's alpha coefficients for the seven subscales ranged from 0.8 to 0.9 indicating a high level of internal consistency. Table 3 provides the alpha values computed for the Organizational Capacity Instrument and each of the seven subscales of the instrument. 
Table 3

Cronbach's Alpha Coefficient for The Organizational Capacity Instrument and Subscales

\begin{tabular}{lc}
\hline \multicolumn{1}{c}{ Scale/Subscale } & Cronbach's a \\
\hline Organizational culture and values & 0.88 \\
Setting priorities for obtaining research to inform decisions & 0.95 \\
Obtaining research evidence & 0.85 \\
Critically appraising research evidence & 0.80 \\
Using research evidence & 0.95 \\
Monitoring and evaluating the impacts of decisions & 0.94 \\
Professional development & 0.90 \\
Total Instrument & 0.96 \\
\hline
\end{tabular}

Findings Related to the Research Questions

Organizational Capacity Instrument. The primary research aim was to explore the extent to which nurse leaders reported components of organizational capacity as present within their respective organizations by using the Organizational Capacity Instrument (Oxman et al., 2009). The total instrument scores ranged from 55 to 180 from a possible range of 0 to 180 and the mean score for the total instrument was $121.78(\mathrm{SD}=27.92)$. The possible and actual range of scores per subscale along with mean and standard deviation are presented in Table 4. 
Table 4

Descriptive Statistics for subscales of the Organizational Capacity Instrument ( $n=43$ )

\begin{tabular}{lcccc}
\hline & Range of Scores & & \\
\hline Subscale (number of items on subscale) & Possible & Actual & Mean & SD \\
Organizational culture and values (6) & $0-30$ & $10-30$ & 23.40 & 5.03 \\
Setting priorities for obtaining research (5) & $0-25$ & $5-25$ & 15.24 & 5.47 \\
Obtaining research evidence (5) & $0-25$ & $5-25$ & 16.85 & 5.51 \\
Critically appraising research evidence (4) & $0-20$ & $4-20$ & 12.13 & 3.74 \\
Using research evidence (5) & $0-25$ & $5-25$ & 16.52 & 4.88 \\
Monitoring and evaluating policies and programmes & $0-25$ & $5-25$ & 17.33 & 4.78 \\
$\begin{array}{l}\text { (5) } \\
\text { Professional development (6) }\end{array}$ & & & & \\
\hline
\end{tabular}

Interpretation of the mean subscale scores. When analyzing the subscale scores, the number of items per subscale was considered and a summative mean was also calculated in order to interpret the mean subscale scores (Burns \& Grove, 2009). In addition to comparing the mean subscale scores, a range was developed to categorize the subscale scores into low, moderate and high categories. Mean subscale scores from 0-15 were classified as low, 16-20 as medium and anything over 20 as high. Using this categorization, one subscale was in the low category (critically appraising research evidence); four subscales were in the moderate category (setting priorities for obtaining evidence, obtaining research evidence, using research evidence and monitoring and evaluating policies and programmes); and two subscales were in the high category (organizational culture and values and continuing professional development). However, because the analysis of subscale mean scores limited the data that could be used to answer the 
research questions, and in order to develop a better understanding of participants' responses to individual items, the individual item mean scores were also calculated.

Interpretation of the item mean scores. The individual item statistics for the Organizational Capacity Instrument are reported in Table 5. The individual item means represent an average of how participants rated a particular item on a scale of zero to five, zero signifying don't know, three signifying neither agree nor disagree and five signifying strongly agree. Therefore, items that had a mean score below three suggested the component was not consistently present in participants' organizations, whereas a mean score of four or greater indicated the component was more consistently available.

In order to compare results across subscales, the mean scores of the last item on each subscale, which measured overall presence of the component, were analyzed. The item measuring overall support was the highest for organizational culture and values supporting the use of research evidence $(4.00, \mathrm{SD}=1.05)$, followed by professional development $(3.63, \mathrm{SD}=$ 1.18), using research evidence (3.56, $\mathrm{SD}=1.08)$, obtaining research evidence $(3.37, \mathrm{SD}=1.16)$, monitoring and evaluating policies and programmes $(3.28, \mathrm{SD}=1.11)$, critically appraising research evidence (3.21, $\mathrm{SD}=1.04)$ and setting priorities for obtaining research $(3.02, \mathrm{SD}=1.19)$. The range of all other items measuring over all support of the component was $3.02(\mathrm{SD}=1.19)$ to $3.63(\mathrm{SD}=1.18)$. Furthermore, a majority of the items on subscales had mean scores that were between 3.0 to 4.0 and therefore indicated participants neither agreed nor disagreed. There were items measuring the provision of time, incentive, resources, expertise or access to experts on all subscales except the setting priorities of obtaining research subscale. These items had mean scores ranging from 2.65 to 3.49 indicating most participants either disagreed or were neutral regarding the availability of these resources and supports. 
Table 5

Descriptive Statistics for items of the Organizational Capacity Instrument $(n=43)$

\begin{tabular}{|c|c|c|c|}
\hline Subscale & Subscale items & Mean & $\begin{array}{l}\text { Standard } \\
\text { Deviation }\end{array}$ \\
\hline \multirow{7}{*}{$\begin{array}{l}\text { Organizational culture and values } \\
\text { [that] support the use of research } \\
\text { evidence to inform decisions }\end{array}$} & a. Our mission or other key organisational documents support evidence-informed decisions & 4.19 & 0.96 \\
\hline & b. Leadership in the organisation supports evidence-informed decisions & 4.33 & 0.81 \\
\hline & c. We are active members in networks that support evidence-informed policymaking or actively & 4.09 & 0.87 \\
\hline & follow the developments and the products of relevant networks & & \\
\hline & $\begin{array}{l}\text { d. We have regular meetings where highly relevant research evidence is discussed in relationship } \\
\text { to decisions }\end{array}$ & 3.35 & 1.29 \\
\hline & $\begin{array}{l}\text { e. Our organisation has committed resources to ensure that research evidence is used to inform } \\
\text { decisions }\end{array}$ & 3.44 & 1.26 \\
\hline & $\begin{array}{l}\text { f. Overall, our organisational culture and values support the use of research evidence to inform } \\
\text { decisions }\end{array}$ & 4.00 & 1.05 \\
\hline \multirow{5}{*}{$\begin{array}{l}\text { Setting priorities for obtaining } \\
\text { research evidence to inform } \\
\text { decisions }\end{array}$} & a. We have explicit criteria for setting priorities for obtaining research evidence & 2.93 & 1.05 \\
\hline & $\begin{array}{l}\text { b. An appropriate mix of people with relevant types of expertise, responsibilities and interests } \\
\text { make decisions about priorities for obtaining research }\end{array}$ & 3.09 & 1.16 \\
\hline & $\begin{array}{l}\text { c. We have an appropriate process for setting priorities for obtaining research evidence } \\
\text { dynamically }\end{array}$ & 3.05 & 1.23 \\
\hline & d. We have appropriate priorities for obtaining research evidence & 3.14 & 1.27 \\
\hline & $\begin{array}{l}\text { e. Overall, our organisation does a good job of setting priorities for obtaining research evidence } \\
\text { to inform decisions }\end{array}$ & 3.02 & 1.19 \\
\hline \multirow{3}{*}{$\begin{array}{l}\text { Obtaining research evidence to } \\
\text { inform decisions }\end{array}$} & a. We have skilled staff to search for and retrieve research evidence & 3.65 & 1.68 \\
\hline & $\begin{array}{l}\text { b. Our staff have enough time, incentive and resources or arrangements with external experts to } \\
\text { find and obtain research evidence }\end{array}$ & 2.65 & 1.36 \\
\hline & c. We have good access to databases such as PubMed and The Cochrane Library and publications & 3.58 & 1.48 \\
\hline
\end{tabular}




\begin{tabular}{|c|c|c|c|}
\hline Subscale & Subscale items & Mean & $\begin{array}{c}\text { Standard } \\
\text { Deviation }\end{array}$ \\
\hline & that report relevant research & & \\
\hline & d. We have good access to national, provincial or local evidence that we need to inform decisions & 3.59 & 1.26 \\
\hline & $\begin{array}{l}\text { e. Overall, our organisation does a good job of obtaining research evidence to inform priority } \\
\text { decisions }\end{array}$ & 3.37 & 1.16 \\
\hline \multirow{4}{*}{$\begin{array}{l}\text { Assessing the quality and } \\
\text { applicability of research evidence } \\
\text { and interpreting results to inform } \\
\text { priority decisions }\end{array}$} & $\begin{array}{l}\text { a. We have skilled staff to evaluate the quality and applicability of research evidence and } \\
\text { interpret the results }\end{array}$ & 3.37 & 1.13 \\
\hline & $\begin{array}{l}\text { b. Our staff have enough time, incentive and resources to evaluate the quality and applicability of } \\
\text { research evidence and interpret the results }\end{array}$ & 2.74 & 1.24 \\
\hline & $\begin{array}{l}\text { c. We have arrangements with external experts to evaluate the quality and applicability of } \\
\text { research evidence and interpret the results }\end{array}$ & 2.81 & 1.30 \\
\hline & $\begin{array}{l}\text { d. Overall, our organisation does a good job of assessing the quality and applicability of research } \\
\text { evidence and interpreting the results to inform priority decisions }\end{array}$ & 3.21 & 1.04 \\
\hline \multirow{5}{*}{$\begin{array}{l}\text { Using research evidence to } \\
\text { inform recommendations and } \\
\text { decisions }\end{array}$} & $\begin{array}{l}\text { a. Our staff have sufficient time, expertise and incentive to ensure appropriate use of research } \\
\text { evidence to inform recommendations and decisions }\end{array}$ & 2.86 & 1.04 \\
\hline & $\begin{array}{l}\text { b. Staff and appropriate stakeholders know how and when they can contribute research evidence } \\
\text { to inform decisions and how that information will be used }\end{array}$ & 3.07 & 1.06 \\
\hline & $\begin{array}{l}\text { c. Our organisation ensures that appropriate stakeholders are involved in decision making and } \\
\text { that they have access to relevant research evidence }\end{array}$ & 3.47 & 1.12 \\
\hline & d. What evidence was used and how it was used is transparent in our decisions & 3.56 & 1.10 \\
\hline & $\begin{array}{l}\text { e. Overall, our organisation does a good job of using research evidence to inform } \\
\text { recommendations and decisions }\end{array}$ & 3.56 & 1.08 \\
\hline \multirow{3}{*}{$\begin{array}{l}\text { Monitoring and evaluating } \\
\text { policies and programmes }\end{array}$} & We routinely consider the need for monitoring and evaluation & 4.00 & 0.93 \\
\hline & $\begin{array}{l}\text { b. Our staff have enough expertise or adequate arrangements with external experts for monitoring } \\
\text { and evaluation }\end{array}$ & 3.23 & 1.21 \\
\hline & c. Our staff have the incentive and resources to conduct/ commission monitoring and evaluation & 3.12 & 1.10 \\
\hline
\end{tabular}




\begin{tabular}{|c|c|c|c|}
\hline Subscale & Subscale items & Mean & $\begin{array}{l}\text { Standard } \\
\text { Deviation }\end{array}$ \\
\hline & $\begin{array}{l}\text { d. Our organisation ensures that appropriate stakeholders are involved in decisions about } \\
\text { monitoring and evaluation }\end{array}$ & 3.70 & 0.99 \\
\hline & $\begin{array}{l}\text { e. Overall, our organisation does a good job of monitoring and evaluation of policies and } \\
\text { programmes }\end{array}$ & 3.28 & 1.11 \\
\hline \multirow{6}{*}{$\begin{array}{l}\text { Supporting continuing } \\
\text { professional development that } \\
\text { addresses important topics and is } \\
\text { evidence-based }\end{array}$} & a. Our staff have enough time for continuing professional development & 3.49 & 1.20 \\
\hline & $\begin{array}{l}\text { b. We have routines to ensure that our staff continue to develop appropriate skills for obtaining, } \\
\text { appraising and applying research evidence }\end{array}$ & 3.12 & 1.16 \\
\hline & c. Our staff prioritise continuing professional development activities that are "evidence-based" & 3.23 & 1.15 \\
\hline & $\begin{array}{l}\text { d. We have appropriate routines for prioritising internal professional continuing development } \\
\text { activities that accommodate the needs of both new and long-term staff }\end{array}$ & 3.30 & 1.19 \\
\hline & $\begin{array}{l}\text { e. We have appropriate routines for deciding whether to support participation in external } \\
\text { continuing professional development activities that accommodate the needs of both new and } \\
\text { long-term staff }\end{array}$ & 3.54 & 1.07 \\
\hline & $\begin{array}{l}\text { f. Overall, our organisation does a good job of supporting continuing professional development } \\
\text { that addresses important topics and is evidence-based }\end{array}$ & 3.63 & 1.18 \\
\hline
\end{tabular}


Facilitators and Barriers to Research Utilization. The secondary purpose of this study was to describe the facilitators and barriers to RU as identified by senior nurse leaders.

Facilitators of Research Utilization. Of the 43 respondents, 60\% $(\mathrm{n}=26)$ answered the question about facilitators. The top three reported facilitators were: developing networks $(\mathrm{n}=18)$, availability of resources $(n=16)$ and organizational support $(n=10)$. Table 6 summarizes the frequency of reported facilitators and each is described in detail below using participant quotes.

Table 6

Facilitators of Research Utilization $(n=26)$

\begin{tabular}{lc}
\hline Facilitator & \# of times reported \\
\hline Networks & 18 \\
Academic links & 7 \\
Research links & 5 \\
Links with other organizations & 2 \\
Links with community partners & 2 \\
Links with professional association & 2 \\
& \\
Resources & 16 \\
Access to an expert & 5 \\
Broad mention of resources & 3 \\
Time & 2 \\
Other & 2 \\
Human & 1 \\
Library & 1 \\
Technology & 1 \\
Financial & 1 \\
& \\
Organizational support & 10 \\
Support from leaders & 4 \\
Culture & 3 \\
Organizational involvement in research & 2 \\
Incentives & 1 \\
Edaff interest & 4 \\
Creating accountability for using research & 3 \\
& \\
\hline & \\
Education and training & 3 \\
\hline
\end{tabular}


Networks. The notion of networks was the most commonly mentioned facilitator among senior nursing leaders. The availability of academic links $(n=7)$ was the most common response in this category and included the need for connections with educational institutions and students (undergraduate, Masters and $\mathrm{PhD}$ ). This category was followed by research links ( $\mathrm{n}=5)$ and included descriptions such as exchange between researchers and policy/decision makers, communication between researchers and end users and having a research department or institute within an organization. Other participants specified networks with professional associations $(n=2)$, other organizations $(n=2)$ and community partners $(n=2)$ to support research use within organizations.

Resources. The second most common facilitator mentioned among participants was the availability of various resources. The most frequently mentioned type of resource was having access to an expert $(n=5)$. Participants added they would value someone: ' $\ldots$ with expertise in evaluating research studies' (participant 13), '...who examines the new literature studies and assess their applicability to their health care setting' (participant 1). Another participant added an 'individual with expertise in adapting research to a local organization, who has a thorough understanding of research methodologies, conducting research and can help staff interpret research findings would facilitate RU among nurses' (participant 14). Three participants did not specify a particular type of resource and two mentioned time. Responses from two participants were categorized as other because they both referred to the use of clinical pathways, medical directives and order sets as evidence-based resources that facilitated RU.

Organizational support. Support from leaders $(\mathrm{n}=4)$ was the most frequently mentioned response in this category, followed by a supportive organizational culture $(n=3)$. A participant explained 'It is about building a culture of inquiry and broadening the perspective of 'research 
utilization' among all staff' (participant 38). Other participants have suggested culture needs to be 'supportive' (participant 1) and 'positive' (participant 10). Two participants also suggested organizational involvement in research, such as nursing advisory councils, facilitate RU and one participant mentioned incentives.

Staff interest. Leaders mentioned 'buy-in' (participant 1), 'receptiveness' (participant 3) 'willingness' (participant 9) and 'engagement' (participant 38) among nurses as a facilitator.

Creating accountability. An interesting category that emerged suggested the need for creating accountability for organizations and nurses to engage in RU. Four participants specified how this accountability might be present within an organization: 'government should include the use of evidence-based research into funding formulas...' (participant 23), 'accreditation process' (participant 34), 'expectations for evidence informed practice embedded in performance competencies' (participant 38) and 'ongoing evaluation of practices' (participant 1).

Education and training. Three participants identified education and training in relation to learning about and promoting the use of research as an important facilitator. For example, one participant identified 'Staff have been sent to attend the EIDM [evidence-informed decisionmaking] Workshop at McMaster as well as the Dorothy Wylie Nursing Leadership Institute to enhance learning and promote the use of research...' as a facilitator for RU (participant 37).

Barriers to Research Utilization. Of the 43 participants in this study, $65 \%$ ( $\mathrm{n}=28$ responded to the open-ended question regarding barriers. The three most frequently reported barriers were: lack of resources ( $n=32$ ), unsupportive organization ( $n=9)$ and $\mathrm{RN}$ attitude/beliefs $(n=7)$. Table 7 summarizes the frequency of barriers and each is described in detail below. 
Table 7

Barriers to Research Utilization $(n=28)$

\begin{tabular}{lc}
\hline Barrier & \# of times reported \\
\hline Lack of resources* & 32 \\
Time & 12 \\
Broad mention of resources & 7 \\
Human & 5 \\
Financial & 4 \\
Knowledge, education and training & 3 \\
Library & 1 \\
& \\
Unsupportive organization & 9 \\
Priorities of the organization & 5 \\
Organizational support / culture & 2 \\
Lack of structure & 1 \\
Lack of leader support & 1 \\
RN attitude/beliefs & 7 \\
Lack of engagement & 2 \\
Lack of interest & 2 \\
Resistance & 1 \\
Hesitancy to engage & 1 \\
Other & 1 \\
Organization size & 6 \\
Constitution of 'evidence' & 2 \\
\hline Some participants reported more than one & \\
\hline
\end{tabular}

*Some participants reported more than one barrier in category.

Lack of resources. The lack of resources was mentioned as a barrier 32 times among nursing leaders. While some participants specified a particular type of resource, others reported a general lack of resources as a barrier $(n=7)$. If a participant mentioned more than one type of resource, each specific resource mentioned was counted individually. Specific resources mentioned among participants included: time $(n=12)$, human $(n=5)$, and financial $(n=4)$. Concerning human resources, one participant added organizations should hire individuals who are truly a good fit for a particular position '... but [they] can be constrained by the need to fill 
vacancies and not wait for the right person' (participant 38). Other leaders expressed financial barriers such as increasing expense of subscription and online access to databases such as Medline (participant 14) as barriers to promoting RU. Three participants mentioned lack of knowledge, education and/or training as barriers and one participant noted an understaffed and under resourced library (participant 31) as a barrier to RU.

Unsupportive organization. Following a lack of resources, an unsupportive organization was the second most common barrier reported by nursing leaders. Five participants mentioned the priority of the organization as a barrier. One participant stated there is 'increased emphasis in promoting RU/EBP at a higher level' (participant 10) and another stated 'too many decisions not evidence based- that are driven from operational need' (participant 48). Another participant echoed this thought and stated 'priority setting for use of best-evidence and resources allocated for same are based on corporate level priorities, not priorities set at the unit-level by the clinical nurses' (participant 32). 'Priorities of senior management' (participant 2) and 'Urgency of issues' (participant 19) were other responses in this category. An unsupportive culture $(n=2)$, lack of senior leadership support $(n=1)$ and lack of structure $(n=1)$ were less frequently mentioned.

Nurse attitudes and beliefs. Seven nursing leaders also noted the attitudes and beliefs of nurses were a barrier for RU in the organization. The leaders specified, resistance $(n=1)$, lack of engagement with research use $(n=2)$, hesitancy to engage in RU (n=2) and lack of interest $(n=2)$ in research use among nurses were barriers. One participant emphasized organizational arrangements supporting RU are present ' ...but we struggle with engagement at all levels' (participant 38). Another leader mentioned that staff nurses did not have interest in training and 
education related to RU (participant 10). In the other category, one participant stated 'most nurses still access the colleague most readily available when delivering care' (participant 32)

Organization size. Six participants who responded to this question cited being a small organization was a barrier to research use. One participant stated bigger organizations usually invest more time and effort in implementing research (participant 7) while another explained 'this study assumes dedicated resources to manage the acquisition of knowledge, that is not a reality in small community facilities' (participant 20). Another participant linked smaller organizations with a lack of available funding and resources to be allocated for research use (participant 37).

Constitution of 'evidence'. A less commonly cited barrier was surrounding the definition of evidence and research. One participant explained ' [the] perception that RCTs are the 'gold standard' and only way of knowing' is a barrier to research use among nurses because it 'limits [the] pool of available research or makes use of other evidence challenging' (participant 14). Another participant echoed this and stated how research is defined is one of the greatest challenges to evidence based decision-making (participant 42).

Other. One participant mentioned 'research that refutes or disagrees with current policy directions/political climate (or vice versa- when the current political demands are not in line with “popular” research opinion)' as a barrier (participant 14). 


\section{Chapter 6: Discussion}

In this chapter, the main findings from this study are compared against what is currently known in the literature regarding research utilization and organizational capacity, with a particular emphasis on senior nursing leaders.

\section{Representativeness of the Sample}

The sample demographic characteristics were challenging to compare to the target population as there are no demographic data collected, by any organization, specific to senior nursing leaders in Canada or Ontario. In 2014, the College of Nurses of Ontario (CNO) reported there were 1, 838 registered nurses employed under the 'senior manager' position category. The types of roles in this category were parallel to the target population of this study. Of these 1,838 senior managers, distribution according to practice sectors was: 453 (community), 463 (hospital), 689 (long-term care), and 233 (other) (CNO, 2014b). In contrast, more than $60 \%$ of participants in this study identified being employed in an acute care hospital and $10 \%$ in long-term care. This discrepancy may be due to several reasons. The higher rates of senior nursing leaders from acute care hospitals may be because NLN.ON and the professional association of nurses in Ontario is connected to a wider network of acute care hospitals as opposed to long-term care. It is also a possibility that there are more senior nursing leaders who work in the acute care setting as opposed to long-term care. In addition, when contacting senior nursing leaders from the salary disclosure list, it was apparent that a majority of those who were emailed were from acute care settings, thus contributing to the higher number of participants in that particular category.

While beneficial, the data from the College of Nurses of Ontario's Data Query Tool (CNO, 2013) relied on nurses self-identifying as senior managers. Without an adequate description of specific roles in this category, this leaves the decision of which category a 
professional belongs to up for interpretation. Furthermore, a restriction of the data query tool includes an inability to set filters related to demographic information or other limits when generating reports. These factors limited exploration of the representativeness of the sample's demographic characteristics. Upon examination, the average age of registered nurses in the general class in Ontario was 45.4 years in 2014 (College of Nurses of Ontario, 2014b). The mean age of the sample in this study (48.7 years, SD 9.9) indicated participants were slightly older than nurses in Ontario. This difference may be attributed to the fact that the study sought senior nursing leaders and this group of professionals had more working experience, and therefore was slightly older than the registered nurses from the general class in Ontario. Without additional demographic information about nursing leaders, determining representativeness of the study sample was challenging.

\section{Reliability Testing of the Organizational Capacity Instrument}

The alpha coefficients for the total and subscale scores of the Organization Capacity Instrument exceeded the minimum set value of 0.70 for a newly developed tool and this suggested good evidence in support of its reliability. The Organizational Capacity Instrument has also been subjected to psychometric testing by Catallo and Sidani (2014) and results of the present study were compared to those reported by the authors. The alpha coefficient values obtained in this study were slightly higher than those reported by Catallo and Sidani (2014) in all but one subscale. The alpha for the critically appraising research evidence subscale was slightly lower than the value reported by Catallo and Sidani (2014).

\section{Discussion of Key Findings}

Comparison of mean scores with Catallo and Sidani (2014). The mean scores obtained for each subscale on the Organizational Capacity Instrument were compared to those reported by 
Catallo and Sidani (2014), as this is one of the only studies, to the knowledge of the researcher, which used the instrument. While mean subscale scores from this current study were comparable to those reported by Catallo and Sidani, there were three subscales in which Catallo and Sidani reported a slightly higher mean. The subscales included: "obtaining research evidence to inform decisions," "assessing the quality and applicability of research evidence and interpreting results to inform priority decisions" and "using research evidence to inform recommendations and decisions." It is important to acknowledge that this difference may be attributable to differences in sample size.

The sample of senior nursing leaders in this study was mainly employed in acute care hospitals and the focus and nature of their work differs from policymakers. The work of a senior nursing leader may have a more clinical focus which is concerned with improving patient outcomes, whereas the work of a policymaker may involve a broader systems level exploration of factors which impact the health of others. Furthermore, while the roles of some senior nursing leaders may also involve the use of research, specific roles might influence the degree to which each relies on research, the type of research that is needed and how and where the research might then be used. These differences may explain why the mean scores for the retrieval, appraisal and use of research subscales, were higher in Catallo and Sidani's (2014) study.

Summary of key findings. This study was one of the first whose purpose was to examine the presence of components of organizational capacity that support research use within organizations in Ontario. When analyzing the results of this study, it was noted that, on average, senior nursing leaders reported a neutral stance on a majority of the items throughout the subscales of the instrument. The components that senior nursing leaders reported as most frequently available in their organizations were organizational culture and values, and supporting 
continuing professional development. In contrast, the components that senior nursing leaders reported as least frequently supported by their organizations were setting priorities for obtaining research evidence and assessing quality and applicability of research and interpreting the results. Interestingly, participants reported the presence of an organizational culture and values that supported RU, yet did not consistently report presence of priorities for obtaining research. Similarly, participants expressed presence of support for professional development, while support for the appraisal, application and interpretation of research was not consistently present.

Furthermore, participants did not consistently report the presence of the provision of time, incentives, resources, expertise or adequate arrangements with external experts on multiple subscales. Some of the results were also reinforced by responses from the open-ended questions in which senior nursing leaders reported the importance of networks, access to resources and organizational support as facilitators to RU and lack of resources, an unsupportive organization and nurse attitudes and beliefs as barriers to RU.

\section{Neutral Findings among Item Mean Scores}

As mentioned above, analysis of the results made it apparent that senior nursing leaders scored a majority of the items approximately in the neutral category on this instrument. To be more specific, of the 36 items on this instrument, over $70 \%(n=26)$ were scored between 3 and 3.70. Furthermore, of all the last items measuring overall support for a component, only one subscale had a mean score that was not in the neutral category. As the number of items that fell into this category was fairly high, there was no pattern that was recognizable. There are several potential reasons why participants may have scored items as neutral.

It is possible that participants were truly unsure about the presence of a particular item because of their role and/or knowledge or level of involvement in the area a particular item 
inquired about. For example, a manager may not be aware of routines for seeking external professional development opportunities and may have to inquire with someone else in the organization. In addition, because of the nature of the items on this instrument that inquired about the organization as a whole, some participants may have felt apprehensive when answering. This raises the possibility of a bias among respondents. Although the instrument was administered in a manner to allow anonymous responses, the possibility that participants felt uncomfortable answering particular items or answering on behalf of the organization and therefore choosing the neutral category cannot be discounted.

Another reason that may have influenced these responses is if participants thought a particular item did not apply to them or their organization. For example, in the subscale that explored organizations doing a good job of using research, items such as the following may not apply to some senior nursing leaders: knowing how and when to use research for decisions, knowing what information would be used, involving stakeholders and being transparent in the evidence that was used and how it was used. Planners and decisions-makers discussed that sometimes even those with authority are not aware of how and if research is used (Bowen et al., 2009). This may also be the case for some participants in this study as some may have exact details around decision-making and knowledge about the processes and procedures of using research, while others may not. Details such as these might have influenced how senior nursing leaders responded to items on this subscale.

The type and size of an organization may be another reason a particular item may not have been applicable to some participants. For example, on the professional development subscale there are items that explore routines for prioritizing internal professional development opportunities and routines for supporting external professional development opportunities. If a 
participant was from a small organization or a particular sector, they may not have the resources and options to provide internal and external professional development opportunities. In cases such as these, a participant may respond to items based on some of the points discussed above. However, it was interesting to note that participants had the option of selecting zero, which meant do not know, yet none of the participants selected this score. This suggests that overall, senior nursing leaders did have an understanding of RU. Therefore, it was interesting to note that participants did not select zero, yet the average mean scores were in the neutral category. While a majority of the items were scored in the neutral category, there were items and subscales that had consistent results in another category and these are discussed below.

\section{Organizational Culture and Values Support the Use of Research}

One of the key findings of this study was that the presence of organizational culture and values that supported the use of research was the subscale with the highest mean score. Specifically, senior nursing leaders reported the presence of items such as mission and key organizational documents, leaders in the organization supporting research use and being part of networks that support evidence-informed policymaking.

Key organizational documents supporting research utilization. Considering the recent move towards integrating research in practice and the national nursing organization endorsing this practice (CNA, 2010), this item scoring as the second highest on this subscale is not surprising. It is possible that the push towards the use of research in practice has facilitated organizations becoming more aware of the importance of RU and subsequently incorporating it as a strategic initiative, and hence why this subscale scored as the highest. Participants in Peirson and colleagues' (2012) study agreed that in order for an organization to support evidenceinformed decision-making, a fundamental shift in the culture of an organization would be 
required. This shift in culture would need to incorporate multiple strategies that endorse a culture of inquiry, critical thinking, learning, risk taking and quality improvement (Peirson et al., 2012). The results of this item might be explained by Peirson and colleagues' (2012) discussion above because, in order for organizations to exemplify support for RU, their organizational documents must indicate the role RU has in that particular organization.

Jeffs and colleagues (2013) also discussed the importance of integrating RU into organizational documents, such as the mission and vision statements. The authors, who reported on an eight-year project that aimed to build nursing research capacity at St. Michael's Hospital in Ontario, revealed that having a shared vision about RU was a key strategic initiative that assisted with engagement among nurses. The engagement of nurses resulted as the vision incorporated the value that nursing research had for the organization, the profession of nursing, interprofessional teams and most importantly for promoting the delivery of high quality care. Results from the open-ended question in this present study also suggested that organizational culture was a facilitator to supporting research use. Various authors have supported the notion of organizational culture being a facilitator to using research (Brown et al., 2009; Ellen et al., 2014; Karkos \& Peters, 2006; Melnyk et al., 2012; Peirson et al., 2012).

Support from leadership. In this study, the item for support from leadership had the highest mean on the organizational cultures and values subscale. One reason for this finding might be because while organizational documents that incorporate RU are critical, their existence alone cannot sustain a culture that supports RU. It is possible that the participants, especially considering their roles, understand the importance of support from leaders and they subsequently make an effort to ensure this support is present. It was also interesting that the sample of this 
study was senior nursing leaders and they were reporting on support from leaders. Therefore, each participant's understanding of who they see as a leader may have influenced the responses.

Other sources also acknowledged the importance of having support from leaders.

McCormack and colleagues (2013) suggested that leaders play an important role in creating and sustaining a culture that promotes evidence-informed healthcare. They suggest that development of a culture that supports research use is facilitated through shared beliefs and values regarding RU among professionals. Participants in Ellen and colleagues study (2014) also explained that when a leader encouraged, facilitated and modeled a particular behaviour it had the potential to become an organizational practice. Furthermore, a qualitative case study established having a clear vision that incorporates an organization's stance on RU and strong leadership that propagates this vision were critical factors for building capacity to support the use of research on a public health unit in Ontario (Peirson, Ciliska, Dobbins \& Mowat, 2012).

As results from the Organizational Capacity Instrument and open-ended questions of this present study also revealed the importance of organizational culture and support from leaders, this further validates the role that these factors have in contributing to organizational capacity that is supportive of RU. Furthermore, this may suggest that when the culture of an organization, which can be manifested through shared value and beliefs about RU, key organizational documents that endorse the use of research and having support from leaders, is supportive of RU, this in turn improves an organization's capacity to support RU.

\section{Setting Priorities for Obtaining Research}

It was interesting to note that while the organizational culture and values that supported RU subscale had the highest mean score in this study, the subscale for setting priorities to obtain research was the second lowest. Having explicit criteria for setting priorities and having a 
process for setting priorities were the two items with the lowest item mean scores on this subscale. It is possible that despite having a supportive organizational culture and values, the use of research is not a priority within organizations, hence explaining the results in this study. Furthermore, organizational culture and values are only one component that impact RU; it is also possible that other factors, such as nurse attitude and beliefs or financial resources, may have influenced the scores on this subscale. It is also important to remember that healthcare culture is often driven by a 'doing' attitude that is dependent on the completion of tasks. A shift to a 'thinkand-do' attitude, which is required for RU, may prove challenging for professionals without adequate support. Bowen and colleagues (2009) refer to this as a "crisis management culture" that is focused on dealing with urgent issues, where research and its use is often a lower priority.

Although not frequent, some participants reported the priorities of the organization as a barrier to RU in this study. Cadmus and colleagues (2008) also reported the priorities of the organization among other goals was a barrier and was often related to financial difficulties within hospitals in New Jersey. Pravikoff and colleagues (2005) also reported the presence of other goals with a higher priority as the number one barrier and concluded this was reflective of disregard about the importance of information resources and nurses access to them.

While some authors reported similar findings regarding the priorities of the organization as a barrier, planners and decision-makers from Bowen and colleagues' (2009) study argued there was uncertainty if evidence was actually used at higher levels. Participants felt that although there was an expectation that decisions at the top were made using evidence, it seemed as though it was not always true. Furthermore, participants felt that decisions were often already made and the evidence supporting particular decisions was sought after the fact, thus creating the notion that politics trumped evidence (Bowen et al., 2009). Interestingly, only one participant in 
the present study mentioned the interplay between research findings that contradict political climate or a political climate that challenges well-known research opinions as a barrier to RU (participant 14). This difference may be due to the fact that Bowen and colleagues study (2009) was conducted with a group of professionals who work in the policy sector.

\section{Assessing the Quality and Applicability of Research and Interpreting the Results}

In this study, the assessing quality and applicability of research and interpreting results subscale had the lowest mean score, indicating participants did not consistently report having support for this component. Some of the lowest scored items on this subscale were: having skilled staff and having arrangements with external experts. One possible reason for this result might be attributed to the fact that RU has only recently become the focus in healthcare, and this has implications on the education that nurses receive regarding the use of research. While nurses may receive some education about critical appraisal and interpreting results of research, the reality of nursing being recognized as a practice-based profession coupled with inadequate educational preparation might help explain these results (Myers \& Meccariello, 2006). Furthermore, it is quite possible that professionals do not feel comfortable or have the skills that are required to assess and interpret research. Many authors have reported that professionals' basic understanding of research and difficulty assessing and interpreting research poses as a barrier (Hauck et al., 2013; Koehn \& Lehman, 2008; Wilkinson et al., 2011).

Having skilled staff. Participants scored the item that explored organizations having skilled staff to evaluate the quality and applicability of research and interpret results as neutral. These results highlight that organizations may not have access to individuals that have expertise in assessing the quality and applicability of research or interpreting the results. In addition, these results might be indicative of the lack of education, training and knowledge to assist 
professionals in building the skills required for this component. The provision of education and training is very important as it has the potential to increase nurses' individual capacity to engage in RU and impact individual attitudes and beliefs regarding RU (Brown et al., 2009).

Yost, Ciliska and Dobbins' (2014) mixed methods study evaluated the effectiveness of a five-day evidence-informed decision-making workshop on knowledge and skills and behaviours, pre-post and 6 months after the intervention. The study revealed a significant increase in knowledge and skills related to evidence use post-workshop (Yost et al., 2014). The study also reported a weak, positive, non-significant correlation between knowledge, skills and behaviour that demonstrated evidence use from baseline to 6-month follow-up. Yost and colleagues' (2014) findings were similar to the results obtained in this study in which senior nursing leaders did not report the presence of skilled staff, and reported lack of knowledge, education and training as a barrier to the use of research. Furthermore, although some senior nursing leaders reported being able to send nurses to various workshops and institutes as a facilitator to research utilization in the present study, the frequency of these responses was limited and might indicate these opportunities do not exist for a majority of nurses. The results of the present study and Yost et al. (2014) provide preliminary support for the need for education and training to ensure nurses have the knowledge and skills that are required to engage in the use of research. From a broader perspective, these results might also reveal that the organization may not be providing the supports that are necessary for nurses to engage with RU.

Similarly, Hart and colleagues (2008) described the implementation of three computerbased learning modules about evidence-based practice. The modules included education about clinical question development, conducting literature reviews, reading and critiquing research articles and examples of implementing research in practice. Knowledge, attitude and skill level 
were measured among nurses before and after the module series. Results revealed that although there were statistically significant differences in knowledge, attitude and skill level postintervention, nurses still need more education about evaluating research (Hart et al., 2008). These findings were echoed by results from Yost and colleagues (2014) who reported that professionals wanted more content related to the critical appraisal of different types of studies. As some participants in the present study also mentioned lack of knowledge as a barrier and considering that the subscale measuring ability to assess the quality and applicability of research was the lowest in this study, the discussion above might suggest the need for tailored education.

Missal and colleagues (2010) also discussed a partnership between a university and healthcare organization that aimed to teach graduate nurses from a framework of evidence-based practice. Based on a recognized need, the authors discussed the use of an Evidence- Based Nursing Practice Committee, which was comprised of staff nurses and faculty and aimed to educate nurses about conducting critical appraisals of the literature. Once the nurses learned how to appraise and integrate research into practice, the authors noted they also began acting as mentors for other nurses on the unit (Missal, Schafer, Halm \& Schaffer, 2010). A strategy such as this would ensure organizations not only have the skilled staff they need, but also create an internal network where nurses might be able to share their knowledge and skills.

\section{Continuing Professional Development}

It was interesting to note that the subscale with the second highest mean in this study explored professional development, yet the lowest scoring subscale related to support for assessing the quality and applicability of research and interpreting results. Considering that some senior nursing leaders reported a lack of knowledge, education and training as barriers to RU and the findings of the subscale above, professional development is critical if nurses are expected to 
be using research. While this subscale had the second highest mean subscale score, an analysis of items revealed all of the scores were approximately neutral. This highlights the importance of analyzing individual item scores as they provide a more in-depth understanding of how participants scored a particular subscale. Some possible reasons for the neutral scores are discussed above. As many of the items on this subscale inquired about the routines needed to provide professionals with professional development opportunities, there was a lack of literature that addressed these specific items. The items that were discussed in the literature are reviewed below.

While professional development opportunities can equip nurses with the theoretical and practical knowledge required to engage in $\mathrm{RU}$, nurses also have to show interest in learning about RU. Participants in the present study reported they neither agreed nor disagreed with staff prioritizing continuing professional development opportunities. Since senior nursing leaders were reporting on behalf of their staff, they may not have felt confident to agree or disagree with this statement. In contrast, a sample of nurses and other health-care professionals from Yost and colleagues' (2014) mixed methods study reported willingness to participate in continuing education as a common theme. When comparing the results of this study to Yost and colleagues' results, the results of this study may indicate issues with nurse attitudes and interest in RU as senior nursing leaders did not consistently report that professionals prioritized professional development activities. The discrepancy between the findings might be attributed to the fact that Yost and colleagues' sampled mainly nurses in public health.

Other items that had overall neutral findings on this subscale related to routines for prioritizing internal development opportunities and providing support for external opportunities. Once again, while there was a lack of literature analyzing routines, Yost and colleagues (2014) 
reported findings related to forms of professional development opportunities. In order for professionals to partake in professional development, the appropriate internal or external processes must be in place. At the end of the educational workshop, Yost and colleagues (2014) asked participants about their preferences for continuing education. Results indicated that because lack of time, geographical restraints and relevance of topics discussed were barriers, participants wanted more than face-to-face sessions. Participants specified where they wanted more education and also specified they would appreciate use of online learning strategies such as webcasts and online learning modules or discussion boards (Yost et al., 2014). While the scope of this study did not extend to exploring reasons why professional development was not a priority, some of the barriers revealed by Yost and colleagues (2104) may assist in explaining why nursing leaders did not consistently agree with this item.

\section{Absence of the Provision of Time, Incentives, Resources, Expertise and Access to Experts}

While the results of this study revealed that a majority of participants neither agreed nor disagreed with items on the instrument, there were items for which participants consistently reported lack of support. A majority of the subscales, with the exception of the setting priorities subscale, had an item exploring the provision of adequate time, incentives, resources, expertise and/or access to experts. Of the six items measuring the presence of organizational support for these components, three item mean scores were in the disagree category and three item mean scores were in the neutral category. A possible reason for these results might be that participants recognized these areas as ones where organizational support is lacking.

The results from the open-ended questions in this study also reinforced the importance of addressing these factors as almost all of the items were mentioned as facilitators and/or barriers. These findings might suggest that while a particular component may be a facilitator in one 
organization, it is also possible it is a barrier in another and this varying status may be dependent on the presence or absence of that component in a particular organization.

Availability of time and resources. One aspect that is critical to the RU is the availability of various different types of resources. On average, senior nursing leaders in this study did not report that their staff had enough time, incentive and resources on multiple subscales of the instrument. Participants also reported lack of resources as the number one barrier, with lack of time, and financial resources being the most frequently mentioned. The finding concerning lack of time as a barrier in this study is also comparable to other studies and may shed light on the reality of nursing practice and the lack of time to engage in RU (Ellen et al., 2014; Liang, Howard, Leggat \& Murphy, 2012; Loke, Laurenson \& Lee, 2014; Peirson et al., 2012; Roe \& Whyte-Marshall, 2012). These results may be explained by an organizational focus on: 'doing' and completing tasks (Peirson et al., 2012) and clinical services (Loke et al., 2014). When the organization creates a culture of this nature, nurses are focused on completing tasks and the focus of their work is on patient care, and it leaves insufficient time to engage in RU.

Another item where some senior nursing leaders in this study expressed lack of support from organizations was access to databases such as PubMed and The Cochrane Library. This finding was further substantiated by comments regarding the lack of financial resources required to invest in access to costly databases by senior nursing leaders in this study. Participants in Pierson and colleagues' (2012) study also reported lack of access to databases and full-text of articles as key barriers to engaging in evidence-informed decision-making. Other sources also acknowledged lack of financial resources as a key barrier to supporting the use of research (Cadmus et al., 2008; Pravikoff et al., 2005). Lack of funding and financial resources not only impact access to research but also library support services. Access to library support may 
addresses the challenges nurses face when searching for research and support the use of research in organizations (Peirson et al., 2012).

Because a majority of the participants in the present study reported working in acute care hospitals with over 50 nursing staff members, these findings were surprising. Organizational characteristics such as these may lead to the assumption that larger organizations have resources to better facilitate access to databases and research, yet the results of this study suggest the opposite. While there is literature that speaks to smaller organizations and subsequent availability of resources to support RU initiatives (Burns, Dudjak \& Greenhouse, 2009; Estabrooks et al. 2003), findings in relation to larger organizations that cite lack of resources are limited (Bowen et al., 2009).

Explanations for this may lie in multiple factors, one of which includes organizational priorities. Findings from this study revealed that senior nursing leaders did not agree or disagree that their organization set priorities for obtaining research. Furthermore, participants in this study also mentioned the priorities of the organization as a barrier to RU. These findings indicate that organizational priorities might interfere with supporting RU. It is possible that if organizations do not value RU or there are competing priorities, the allocation of resources may be hampered. Participants in Ellen and colleagues (2014) study also suggested that organizations become accustomed to practicing a certain way and often show resistance or negative attitudes towards change. This may highlight that priorities for RU or allocation of resources to support RU are not the only factors to consider, the organization and leaders must be willing to engage in RU and accept the associated changes that may come about as a result (Peirson et al., 2012).

Availability of incentives. While only one participant in this study mentioned the availability of incentives as a facilitator, responses for items that incorporated this component 
revealed that overall participants did not report it was present in the organization. Perhaps senior nursing leaders did not mention incentives more frequently because there are resource implications associated with providing incentives. It is also possible that organizations are not engaged in RU; therefore a need for offering incentives might not even exist. Chummun and Tiran (2008) recommended the implementation of a rewards system to enhance nurse interest and increase engagement in RU. While one nursing leader mentioned the use of incentives as a facilitator, another suggested the need to build RU into performance competencies for staff. Creating accountability for engaging in RU was also mentioned among the top priority items among participants in Ellen and colleagues (2014) study as a means to support the use of research. In addition to creating accountability through integrating RU into performance competencies, research has also alluded to the benefits of offering incentives as a means to facilitate RU (Liang et al., 2012).

Another reason why it is important to explore the availability of incentives is the impact it has on motivation to engage in the use of research (Chummun \& Tiran, 2008). A review of 25 research articles from Europe and America concluded that nurses report "... a lack of time, ability and motivation to appraise research reports and adopt findings in practice" (Chummun \& Tiran, 2008, p. 327). These results suggest it is important to analyze the availability of incentives, as there may be an impact on motivation.

Access to experts. Multiple items on the instrument also investigated access to experts. It was interesting to note that access to experts was the most frequently reported facilitator under the category of availability of resources. While results for items that included this component revealed access to experts was not present in organizations, multiple participants expressed that having access to someone with expertise in critical appraisal or with knowledge and skills related 
to $\mathrm{RU}$ as a facilitator. While the item results did not reflect what was present in organizations, open-ended responses revealed what was desirable by senior nursing leaders. This may be one reason that participants responded this particular way. Another reason why participants might not have reported having access to experts is lack of resources to hire someone with expertise or difficulty finding professionals with this type of knowledge and skills.

As numerous studies report that front-line staff nurses lack the education, training, knowledge and skills required for engaging in $\mathrm{RU}$, the role of an expert can include: assisting nurses in the search, retrieval, appraisal and application of research, interpreting and adapting results of research to a local context, and acting as a resource for research related questions (McCormack et al., 2013). Similar to the results of this study, research has also supported the use of experts in facilitating RU among nurses and also emphasized the vital role they play in sustainability of these practices (Brown et al., 2009; Melnyk et al., 2012). While come sources discussed the benefits of experts, McCormack and colleagues' (2013) literature review, which used the term change agents instead of experts, concluded their effectiveness at promoting evidence-informed healthcare was not currently established.

One reason why McCormack et al. (2013) might not have been able to conclude effectiveness of experts could involve issues regarding access. To be more specific, a cyclic situation whereby effectiveness of experts cannot be established because they are not available might complicate the issue. Despite mixed findings regarding the benefits of experts, nurses in Melnyk and colleagues' (2012) study validated this point as they explained access to experts in healthcare settings was fairly limited. It might be important to have experts in organizations as their role is not merely to provide support related to the technical skills of research use, but also 
to build individual capacity by motivating and encouraging nurses to partake in research activities and fostering long-term sustainability (Smith \& Donze, 2010; Squires et al., 2012). Facilitators and Barriers to Research Utilization

A majority of the facilitators and barriers that were reported by senior nursing leaders in this study have been discussed throughout this chapter. Therefore, this section will highlight one key facilitator and barrier, both of which have not been discussed elsewhere in this chapter.

Networks as a facilitator. The availability of various types of networks was the number one reported facilitator to RU among senior nursing leaders in this study. Leaders mentioned various types of networks, but the connection between all examples was the presence of a partnership or connection of some sort. For example, some participants expressed links with academic institutions, students in varying educational programs, between researchers and policy/decision makers were facilitators to RU. In addition to the responses from the open-ended question, some items on the Organizational Capacity Instrument also explored the presence of networks. The organizational cultures and values subscale included an item that explored organizations being part of active networks that support the use of research or actively following the developments and products of relevant networks. Other items also explored the presence of arrangements with external experts for the professional development and using research subscale and the involvement of stakeholders in decision making from the monitoring and evaluation subscale. While responses to the open-ended questions revealed networks as a facilitator, average scores for these items were in the neutral category. In addition to the reason for neutral scores that were discussed above, these findings may have resulted from the instrument failing to provide more descriptions or examples of these various types of networks. 
Nurses in Melnyk and colleagues' (2012) study also reported networks as a facilitator for implementation of evidence-based practice; however it was seventeenth among a list of 18 facilitators. The difference between the results of this study and Melnyk and colleagues (2012) may be due to their sample of nurses. Front-line nurses may answer questions about facilitators differently than senior nursing leaders as they are focused on clinical practice. On the other hand, senior nursing leaders in this study might have responded based on a broader systems-level perspective. Ellen and colleagues (2014) also mentioned that establishing links between researchers and knowledge brokers are key priority areas for organizations as this can support evidence-informed decision-making.

In another study, Ellen et al. (2013) noted the importance of developing networks with researchers and opinion leaders outside the organization. This particular example of networks was the least frequently reported among nursing leaders in this current study. Similarly, because Ellen and colleagues' sample (2013) consisted of senior management, library managers and knowledge brokers, the influence of differing roles and responsibilities might have influenced the results of their study a particular way. The need for links with educational institutions was the most cited example in this current study. Although this was not frequently mentioned in the literature, Drenkard (2013) suggested the benefit of links with educational institutions include organizations gaining access to resources and libraries that the institution has. Regardless of the type of networks discussed, some of the discrepancies between findings from the instrument, open-ended question and literature may warrant a need to explore this factor further.

Nurse attitudes and beliefs as a barrier. While this chapter includes an in-depth discussion regarding the lack of protected time, incentives, resources and expertise as barriers to $\mathrm{RU}$, authors argue that even when nurses have some of the factors mentioned above, they are not 
engaged in RU (Gerrish et al., 2008; Thiel \& Ghosh, 2008). This notion might suggest that there is more involved when analyzing limited use of research among nursing professionals. One possible explanation for limited use of research by nurses, despite the concerns mentioned above, may be due to attitudes and beliefs towards RU. This was also reflected in responses to the openended questions in which nurse attitudes and beliefs were the third most frequently mentioned barriers to RU. Brown and colleagues (2009) validated the importance of addressing attitudes as they reported a statistically significant correlation between attitudes and knowledge related to evidence- based practice among nurses at an academic medical center in California.

Pravikoff and colleagues (2005) reported when organizations perceived that nursing staff was not eager to pursue evidence-based practice, it posed as a barrier to promoting its use. Part of the issue with lack of nurse engagement may be due to a lack of confidence or skills required to engage in RU, which makes nurses less likely to consult research when information is needed (Cadmus et al., 2008; Gerrish et al., 2008; Pravikoff et al., 2005; Thiel \& Ghosh, 2008). The results of the present study have also revealed low scores and neutral findings on the subscales measuring the access, appraisal and application of research and results comparable to the literature regarding nurse attitudes and beliefs as a barrier to RU. The findings of the present study coupled with the review of other sources substantiate the need for strategies that improve nurses' attitudes and beliefs towards RU. Furthermore, because attitudes and beliefs may be impacted by other factors, it may be beneficial for organizations to explore the source of these feelings in order to better address them. In addition, while the present study highlighted the need to explore factors other than individual attributes, these findings reveal that this particular attribute is one that cannot be overlooked. Organizations and senior nursing leaders should explore what might be contributing to nurses' attitudes and beliefs towards RU. 
While some sources link attitudes and beliefs to a lack of knowledge, skills and confidence to engage in RU, a literature review revealed the most cited reason for lack of interest in RU was related to the lack of a research friendly environment (Chummun \& Tiran, 2008). More specifically, the authors revealed that the lack of research activities and facilities that fostered an interest impacted attitudes about RU (Chummun \& Tiran, 2008). This can further be linked to the importance of creating a culture and values that support RU within organizations. And because the literature has linked attitudes and beliefs to multiple other components of RU, this stresses the importance of exploring nurse attitudes and beliefs.

\section{Summary}

Overall, the results revealed that a majority of the items on the subscales of the instrument were scored in the neutral category. In addition, the two components that senior nursing leaders reported as being present were: organizational cultures and values that support the use of research and support for continuing professional development. Interestingly, the components that senior nursing leaders reported as least supported by their organizations were setting priorities for obtaining research evidence and assessing quality and applicability of research and interpreting the results. Furthermore, participants in this study did not report organizational support related to the provision of sufficient time, incentives and expertise or resources and access to experts on multiple subscales of the instrument. These findings highlight and substantiate the themes discussed throughout this chapter and suggest critical learning points for organizations.

While the results provided valuable discussion points, it is important to acknowledge that the magnitude of the mean subscale scores reflected responses that were on the middle to higher end of the scale, suggesting that overall the participants thought their organizations were doing 
fairly well with respect to presence of these components. The results of this study highlighted key points that contributed to a preliminary understanding of the factors of organizational capacity that senior nursing leaders report as present in their organizations.

\section{Study Limitations}

While this study contributed to the generation of knowledge regarding the components of organizational capacity that support RU among nurses, there were limitations present which should be taken into consideration when interpreting the results of this study. The remainder of this chapter will include a discussion of the limitations related to the sample, recruitment and measurement.

Sampling. This study targeted senior nursing leaders and relied on the use of convenience, snowball and purposive sampling methods. As every member of the target population does not have an equal chance of being included in the sample, some may consider these sampling methods weak and consequently a limitation of this study. Furthermore, Burns and Grove (2009) suggest this decreases the likelihood of having a sample that is representative of the target population. Through the use of these sampling methods, issues with the researcher's ability to generalize the findings beyond those in the study might also be raised. For example, because participants from the Ministry of Finance salary disclosure list were limited to those who earn above a certain income, this may impact the generalizability of results. Despite these limitations, it is important to consider that senior nursing leaders are employed in various settings, across multiple geographic areas and there is no centralized group of all nursing leaders in Ontario. Therefore these strategies facilitated access to a group of nursing professionals that are otherwise difficult to access. 
Burns and Grove (2009) add another limitation of convenience sampling in particular is the inability to control for biases which impact generalizability of the findings and representativeness of the sample. However, through careful thought of the sampling criteria, specific inclusion and exclusion criteria were determined to control for possible biases. While these limitations exist for the chosen sampling methods, it is important to consider the methods are appropriate for a descriptive study.

Recruitment. During the course of this study, multiple recruitment strategies were needed and while they were beneficial, there were associated limitations. One such strategy was the use of the Ontario Ministry of Finance (2014) salary disclosure list. Issues regarding nonresponse bias, being unable to obtain contact information for nursing leaders and refusal rates were apparent. Regardless of the reason why nursing leaders did not participate, it ultimately resulted in a loss of potential participants. Furthermore, the effect of non-response bias is also a possibility as the responses of leaders who participated in this study may have been different from those who did not participate. However, despite these drawbacks, the use of multiple recruitment strategies was a strength for this study as it allowed the researcher to include a broader range of participants from multiple types of settings, with a variety of backgrounds and level of experience and this strategy facilitated the increase in sample size for this study.

Another limitation of the recruitment strategies was the inability to calculate response rates for this study. For the NLN.ON interest group newsletter, Facebook and LinkedIn page it was not possible to determine how many participants received or opened the advertisement compared to those who agreed to participate and actually participated. Furthermore, because the exact number of senior nursing leaders in Ontario is unknown, it was difficult to compare the results of this study. While this raise concerns of sampling bias and impacts representativeness of 
the study sample to the target population, these recruitment strategies were necessary to sample senior nursing leaders (Burns \& Grove, 2009). Furthermore, the study not only met but also exceeded the target sample size set for this study.

In addition to using multiple recruitment strategies, the data collection time frame for this study also needed to be extended. Due to the fact that the enrolment in this study was initiated in the summer of 2014, this extension was necessary. The timing of enrolment may have coincided with holiday and vacation schedules of nursing leaders and hindered the effectiveness of recruitment strategies. If the study was conducted at a different time, a higher response rate and larger sample size may have been attainable. Furthermore, an increase of the data collection time frame may impact the study findings, as the researcher may not know if anything happened during the course of the study to influence the results. This may result in a possible threat to internal validity related to history (Burns \& Grove, 2009). However, considering the study design was descriptive and the time increase was minimal, the possibility of time influencing the results is very small and the benefit of increasing sample size was more important. Moreover, had the study been terminated according to the original timeframe, the number of participants in this study would be less than that targeted and would impact generalizability of findings.

Measurement. Although the use of open-ended questions permitted self-expression and richness of detail regarding the facilitators and barriers to RU, there were accompanying limitations. Although the participants had a choice in answering these questions, it may be possible that only the participants who were passionate about the topic or had something to contribute responded to the questions. The individuals who responded may have felt strongly about certain facilitators and barriers and voiced their opinions, with varying degrees of detail and this could have impacted the answers received. Furthermore, considering the participants 
completed the Organizational Capacity Instrument immediately before the open-ended questions, their responses may have been impacted by the content of the instrument. While these types of limitations may have existed in this study, it is also important to consider the contribution these responses had to the study and that the nature of the questions permitted participants to respond in any manner, without being limited to a set of pre-determined questions and responses.

Validity testing of the Organizational Capacity Instrument. It is also important to note that validity testing was not completed for the Organizational Capacity Instrument in this study. While validity testing is important to determine if an instrument actually reflects what is being measured (Burns \& Grove, 2009), it was not conducted because the focus of this study was not on the psychometric evaluation of the instrument. It would be beneficial for future researchers to incorporate validity testing for psychometric evaluations of this instrument.

Unsystematic threats to internal validity. When analyzing the amount of time that it took participants to complete the study through Opinio, it became apparent that the length of time varied greatly. The shortest amount of time for completion was 4-5 minutes and the longest time was 82 minutes. The amount of time it took to complete the survey may have been dependent on the schedules of the senior nursing leaders, and subsequently may have impacted the results of the study. For example, if a participant was having a particularly busy day and did not allocate sufficient time to complete the survey, it may impact how they answered responses. In contrast, some of the longer times of completion may be attributed to leaders becoming busy and having to come back to the survey. This variability can lead to unsystematic threats to internal validity, which is random in nature and defined as “...variability within individuals and/or groups of individuals" (Martella, Ron Nelson, Morgan and Marchand-Martella, 2013, p.41). This threat has the possibility of influencing internal validity of the study, but Martella and colleagues explain 
that the impact of such elements is often minimal and these threats are "...a fact of life that cannot be totally eliminated or accounted for" (2013, p. 41).

Missing data. Another limitation of this study was the missing data from the Organizational Capacity Instrument. Missing data has the potential to impact the internal validity of a study and the risk is greater when the sample size of a study is small (Burns \& Grove, 2009). However, it is important to note the amount of missing data was quite small in this study. Furthermore given the organization of the subscales on the Organizational Capacity Instrument, the researcher chose an imputation technique to handle the missing data and this also facilitated analysis of a complete data set while preserving sample size of the study.

Study design. Another critical factor to consider is the descriptive-quantitative design of this study. While a descriptive study design many have limitations in terms of inability to conclude differences, relationships or make predictions, it is important to consider the topic of interest. More specifically, if the degree of existing knowledge about a particular topic is limited, descriptive research is beneficial and also has the potential to build the foundation for future research efforts (Burns \& Grove, 2009). In the case of research utilization, although this area is not novel in the nursing profession, there is limited research regarding organizational capacity and the role it may play in impacting RU, in Ontario. For this reason, a descriptive design was considered appropriate for this study. 



\section{Chapter 7: Summary, Implications and Conclusion}

\section{Summary}

In recent years, research utilization among healthcare professionals, nurses in particular, has gained considerable attention. The use of research by nurses is critical to the delivery of care that is safe, improves patient outcomes and based on the best available research. Engagement in the use of research necessitates that professionals have the knowledge and skills required to search, access, critically appraise, interpret and apply research to their local context (Oxman et al., 2009). Despite documented benefits of using research in practice, many nurses continue to be disengaged in the process. Initial exploration of reasons contributing to the disengagement led authors to believe the individual attributes of nurses, such as years of experience, level of education and attitude towards research use impacted a professionals decisions to use research in practice (Estabrooks et al., 2003; Scott-Findlay \& Golden-Biddle, 2005). However, research has revealed that with the exception of nurse attitudes and beliefs, there was little to suggest that individual attributes impacted RU (Brown et al., 2009; Cadmus et al., 2008; Estabrooks et al., 2003; Kocaman \& Lash, 2011; Melnyk et al., 2012).

These findings led authors to explore other factors that may have been contributing to lack of nurse engagement. This shift diverted attention from the individual attributes that impact RU to the environment in which nurses' work and are expected to use research. While the act of RU is dependent on the nurse, the organization has an important role in facilitating RU (Cadmus et al., 2008; Scott-Findlay \& Golden-Biddle, 2005; Melnyk et al., 2012). In this sense, it becomes important to explore an organizations' capacity to support RU among nurses. Furthermore, as senior nursing leaders are in a position of power and authority and can influence change within an organization, their involvement when exploring organizational capacity and the 
facilitators and barriers of RU is critical. Moreover, there is a lack of research exploring the presence of components of organizational capacity that support RU in organizations within Ontario.

A descriptive, quantitative study was conducted to explore the extent to which senior nursing leaders in Ontario reported the presence of components of organizational capacity that can support RU and examine the facilitators and barriers to RU. The SUPPORT series and Organizational Capacity Instrument by Oxman and colleagues (2009) was used as a guiding framework to explore the presence of seven components of organizational capacity. This study found that the two components that senior nursing leaders reported as most present in their organizations were organizational culture and values that support RU and support for continuing professional development. In contrast, the two components that participants reported as least frequently supported by their organizations were setting priorities for obtaining research evidence and assessing quality and applicability of research and interpreting the results. While the majority of mean item scores were in the neutral category, results concerning the provision of sufficient time, incentives, resources, expertise or arrangements with external experts on various subscales were consistently reported as being unavailable within organizations. Furthermore, the facilitators and barriers reported by participants substantiated the results from the instrument.

\section{Implications}

As this was the first study to explore the extent to which components of organizational capacity that support RU were present in organizations and identify associated facilitators and barriers to research use, the results provide a preliminary understanding of how organizations may be contributing to RU among nursing professionals. Implications of the results for nursing practice, education, research, policy and theory will be described next. 
Implications for nursing practice. The results of this study emphasize the importance of an organizational culture that is supportive of and facilitates RU among nurses. The need to create an environment that values and promotes the use of research in practice has been supported by a review of the literature and findings from this study. To begin with, organizations and leaders must demonstrate that RU is valued at all levels in the organization and also display that nurses are a key part of this movement. Thompson suggests the need to "develop a climate where research is valued, seen for its worth and a routine part of activity in the organization" (2003, p.143). If senior nursing leaders endorse the need for a culture of inquiry and they promote critical thinking among nurses, this can assist in the development of a culture that is supportive of RU. The development of such a culture can be facilitated through shared beliefs and values regarding $\mathrm{RU}$ among professionals. Implementation of a culture of this nature requires considerable support from leaders. McCormack and colleagues (2013) stressed the need for leaders who have an optimistic attitude and well-established respect, credibility and trust to be able to influence employees. This is critical if hesitancy to engage in RU or lack of interest among nurses is to be addressed. One of the ways in which organizations and leaders can demonstrate support and value for RU is the allocation of resources.

Availability of resources is critical because without access to the necessary resources, barriers that make it challenging for nurses to be engaged in RU are created. The findings of this study revealed that overall nursing leaders disagreed that the provision of time, resources and incentives were present within organizations. Research has suggested that it is important for leaders to secure dedicated time for professionals to engage in the use of research as this exemplifies support from management and the organization (Bowen et al., 2009; Peirson et al., 2012). In addition to securing time to engage in RU, researchers have also suggested the need for 
technical infrastructures that support RU and ensure nurses have access to research (Ellen et al., 2014; Gerrish et al., 2012; Melnyk et al., 2012). Access to databases or an internal library system is key to providing this support. These are vital resources that support nurses' access to research. Nursing professionals must know the organization supports RU by creating an avenue for assessing research. One strategy that organizations may benefit from is the addition of librarians who can assist professionals in searching for research and improving access to databases and research articles. Senior-nursing leaders can facilitate the coordination of an internal library system that is critical if nurses are to be engaged in RU (Peirson et al., 2012).

While the aforementioned suggestions regarding time and infrastructure resources may create challenges for organizations that operate under a restrictive fiscal climate in healthcare, there are alternative solutions. While feasibility of changes to the allocation of resources may limit what organizations can do to support nurses' use of research, one strategy may involve creating a new role to support RU or investing in current staff members so they may ultimately transition into an expert role. Organizations may benefit from the integration of an expert whose role is to plan and coordinate organization-wide knowledge, interest and skills in RU. Peirson and colleagues (2012) describe the role of a Manager for Education and Research in initiating staff training, coordinating educational services with the library and also focusing on the areas in which nurses needed assistance. Peirson and colleagues (2012) reported the manager not only increased knowledge and skills through the provision of education, training and library support but one of their main responsibilities included creating an organization-wide structure for evidence-informed decision-making. These findings highlight the importance of having the appropriate structures and processes, and an individual with expertise in RU, to support the use of research. 
While the factors mentioned above require substantial support and coordination from senior nursing leaders, the integration of research use must be a systemic, organization wide effort. Senior nursing leaders are in a key position to propose a process for the implementation of RU within organizations. An important part of this process is to develop strategies that integrate research into the existing processes within an organization as opposed to marketing it is an additional task for nurses to complete (Bowen et al., 2009). Examples may involve the development of decision aid tools or creating algorithms for particular aspects of care (e.g. discharge planning, routine mental health screening and more). Strategies such as these provide practical examples of how research use can be beneficial to nurses and their practice.

Another part of this process is creating accountability for nurses to be engaged in RU. Bowen and colleagues (2009) suggested a redefinition of roles was required to inform nurses what was expected of them, but also assuring them they had support from the organization and hence the need to redefine roles. Using this approach, nurses are held responsible and accountable to be engaged in the use of research but they are also provided the support that is needed to meet these expectations. The use of an accountability framework may consist of the integration of professional development credits in practice, encouraging nurses to proactively seek external professional development opportunities or embedding engagement in RU within performance reviews of nurses. Furthermore, without the use of an accountability framework it may be difficult to engage nurses and they may be unclear about the expectations and possibilities to be involved. It is important to note that the organization should not be expected to address the individual attributes of nurses that may or may not impact RU. Professional standards, which guide nursing practice, have outlined that the use of research is a professional 
responsibility for all nurses. Therefore, while the organization and senior nursing leaders can support RU, it should not be expected that they address the individual attributes of nurses.

Once an organization has a process and culture with skilled staff and the availability of resources, sustainability of practices and maintaining nurses' interest and engagement is key. While the factors mentioned above can increase an organizations' capacity to support RU, it is also important to have strategies, such as a research symposium/day, that showcase examples of RU within the organization and share success stories with employees. Melnyk and colleagues (2012) stated a 1-2 day research workshop would not contribute to sustainability of research efforts. Similarly, Krugman (2010) suggested initiatives to promote RU needed to be constant, as opposed to a one-time initiative. Krugman (2010) added it was important to raise awareness about and build interest in RU, then increase involvement in the use of research and build individual capacity. Activities such as those mentioned above would promote communication of successes, sharing of lessons learned and also create an opportunity to build formal and informal networks between colleagues. Another integral component to maintaining nurses' interest and engagement and building individual capacity is the recognition of the nurse for his/her achievements. Hauck and colleagues (2013) stressed the importance of incentives in highlighting achievements and also conveying a message to nurses that the organization places importance on and rewards nurses who engage in RU. While the type of incentive may vary among organizations, it is important that senior management teams understand the importance of and place value in rewarding professionals for their involvement in research.

While organizational support is critical to supporting engagement in $\mathrm{RU}$, this study also revealed that senior leaders identified nurse attitudes and beliefs as barriers to RU. Seniornursing leaders and organizations may create a process for RU and also provide the support 
necessary, however these efforts cannot be successful without engagement from the nurse. Implications for nurses involve being more active in seeking opportunities to be engaged in RU. Because nurses are in the clinical environment most of the time, they are in an optimal position to identify areas where change is needed and bring these to the attention of leaders so a process that involves the use of research can be initiated. While the role of senior-nursing leaders is to promote the value of RU at various levels of the organization and create a culture that is supportive of RU, their efforts are meaningless if nurses are not engaged in or show interest for RU. If organizations and senior- nursing leaders witness interest and engagement this may substantiate a need to continue to provide organizational support for RU. In contrast, if nurses are not engaged in RU, organizations may question the need for providing support or increasing capacity to provide support to nurses.

Implications for education. Another area that is consistently reported as a barrier in the literature and supported by the results of this study is nurses' struggle with assessing the quality and applicability of research, and interpreting the results for use. Consistent findings highlight this area as a concern and point to the need of providing nurses with educational opportunities aimed at enhancing RU skills. While this study had an organizational focus and was interested in RU among nurses, education and training related to research and RU should ideally begin at an undergraduate level. A systemic integration of understanding research, various study designs and skills in assessing the quality of research are critical while nurses are in university or college. If these skills are incorporated into undergraduate education, this may ease feelings of hesitancy while also building the confidence and interest that can result from being knowledgeable about research. This might be related to the fact that in addition to promoting technical skill building, education may influence attitudes and beliefs, and subsequent engagement with RU. Brown and 
colleagues (2009) reported a statistically significant correlation between knowledge and attitudes toward RU, further emphasizing the notion that educating nurses results in feelings of confidence, which are directly associated with increased knowledge and skills regarding RU.

In addition to increasing confidence and decreasing feeling of hesitancy, Thiel and Ghosh (2008) reported that level of knowledge about evidence-based practice was significantly positively correlated with the level of nursing education, indicating that knowledge scores increased as level of education did. While education and training about research and the use of research in practice is incorporated in academic preparation, part of the responsibility of providing nurses with professional development opportunities rests with the organization. This is especially true if there is an organizational expectation that nurses be involved in RU. Barnsteiner and colleagues (2010) discuss the use of fellowships in which nurses apply for oneyear fellowships, which involves monthly meetings. During the fellowship, nurses receive ongoing education about research, research methods and use of research in practice. The fellowship program also provides nurses the opportunity to design and implement a research project. Strategies such as fellowships can foster interest among RNs, yet also provide support throughout the process to address issues such as lack of knowledge. In Ontario, the Registered Nurses Association of Ontario also offers members an opportunity to apply for an Advanced Clinical Practice Fellowship (http://rnao.ca/bpg/get-involved/acpf). Organizations can look into promoting and securing nurses the time to engage in such initiatives, especially because opportunities of this nature often include financial support and access to a wide network and resources. In addition to feeling supported by the organization, strategies such as this also connect nurses to those with expertise in using research. 
Although an external fellowship offered by a professional association is one strategy that promotes knowledge building and skill development, organizations can also introduce internal initiatives. Peirson and colleagues (2012) discuss the use of journal clubs and lunch and learn sessions that are geared to the needs of staff while also promoting internal collaboration. These clubs can be based on the needs of the staff in a particular organization and may also assist in creating an environment where individuals are comfortable with one another, thus creating a positive learning environment. A key area that nurses continually struggle with is the critical appraisal of research and studies have suggested the need for critical appraisal training (Peirson et al., 2012; Yost et al., 2014).

Participants in the study by Yost and colleagues (2014) participated in an intensive evidence-informed decision-making workshop and when questioned about need for continuing education after participating in the workshop, a consistent theme was the need for more content about critical appraisal of different types of studies. Health-care professionals who participated in Yost and colleagues' (2014) workshop had the opportunity to work in small group sessions that focused on searching for, accessing and critically appraising research in a team environment. For example, participants worked in groups of two to three and compared responses to critical appraisal criteria and then presented their findings to a larger group. Strategies such as this use an active teaching and learning approach while having the added benefit of having peer support throughout the process. Similarly, Peirson and colleagues (2012) discussed the use of a Critical Appraisal club as a strategy to allow professionals with common interests to meet regularly and develop knowledge and skills related to an area where there is a need, all in a supportive team environment. Senior-nursing leaders could play a role in initiating and promoting these groups 
until they have the capacity to run independently and it would ultimately provide support in the most needed area and improve engagement with the use of research.

While multiple studies have concluded that the delivery of an educational intervention has a positive impact on knowledge, beliefs and attitudes towards RU (Aitken et al., 2011; Bartlet et al., 2011; Hart et al., 2008; Melnyk et al., 2010; Yost et al., 2014), a mixed methods study also challenged the effectiveness of this approach. Loke and colleagues (2014) concluded the need for a significant shift in focus regarding educational training and suggested there should be a greater emphasis on providing nurses with opportunities to get involved in the research process. Providing nurses with an opportunity to apply what they have learned into practice might be more effective than participation in a workshop in which the knowledge gained remains stagnant. This shift in perspective regarding the use of typical didactic educational strategies as opposed to interactive educational strategies may involve the role of leaders.

Senior nursing leaders may be critical in providing resources to nurses who are interested in individual professional development opportunities. For example, leaders might provide information about the McMaster Evidence Based Health Care Workshop (McMaster University, 2015) or the learning resources provided by The National Collaborating Centre for Methods and Tools (NCCMT)- Evidence Informed Decision Making Series (NCCMT, 2012). The use of these independent learning strategies could be provided to those interested, built into orientation for new staff, provided as part of a professional develop opportunities list or built into performance appraisals of nurses to increase accountability. Whether organizations facilitate group education/training or provide support for individual professional development opportunities, the message conveyed regarding support from the organization is identical. This support may also assists in creating and sustaining a culture that values and promotes RU among staff. 
Implications for research. This study used the Organizational Capacity Instrument (Oxman et al., 2009) to explore the extent to which senior nursing leaders reported components of organizational capacity as being available in their organizations. While the tool has been subjected to preliminary psychometric testing and also demonstrated an acceptable level of internal consistency reliability in this study, it is still a fairly new tool. Additional testing of this tool is needed with larger sample sizes to further assess its psychometric properties (Catallo \& Sidani, 2014).

While this tool was developed for policymakers, its use in this study was critical as there are no tools that explore organizational capacity from a general healthcare lens. One of the limitations of using a tool that is geared to a specific profession includes the possibility of item content not being applicable to groups other than for which the tool is intended. Some of the items on this tool were very specific to policymakers and may not have applied to the work of a professional in a clinical setting, thus influencing the results of the study. Furthermore, while there has been some preliminary psychometric evaluation of the tool, further validation of the Organizational Capacity Instrument would be beneficial as it is a fairly newly developed tool. The psychometric evaluation of the instrument in this study revealed that while there was good evidence to suggest reliability of the subscales, Cronbach's alpha values were on the higher end and might indicate redundancy of items measuring a particular component of organizational capacity (Tavakol \& Dennick, 2011). Future research may focus on adapting and pilot testing the existing tool to further enhance its psychometric properties and usability across different sectors in healthcare. Adaptation of the tool may include deleting items that contribute to redundancy, adding items that aim to capture more information regarding the availability of different types of 
resources or adding items that explore the involvement of individual attributes and components of organizational capacity.

Future research might seek to examine relationships between organization type and/or size and support for RU with a larger, varied sample. Organization size was mentioned as a barrier in this study and warrants exploration of inequalities that may exist. Thus, future research may seek to compare the differences within types of organizations, comparing supports for RU within a large teaching hospital as opposed to a small community hospital. Research such as this could help organizations complete a self-assessment of their strengths in supporting RU, exploring areas for improvement and also the current status of engaging with RU in their respective organizations.

Implications for policy. Senior-nursing leaders in this study reported barriers surrounding funding and this has significant implications from a policy perspective. Some of the issues related to funding included impact on resource allocation and the inequities experienced by smaller organizations. These findings point to the need for organizations to connect with policymakers and researchers to articulate and empirically substantiate the issues experienced by organizations where funding is a challenge. Coupled with an increasing need for organizations to be accountable for their decisions and allocation of resources, this warrants the need for organizations to explore how funding and resource are used, where there is a deficit, why this deficit might be occurring and the importance of reducing inequities. It is also critical that organizations make a convincing argument that supports the need for RU and funding that supports these efforts. Once again, senior-nursing leaders might play a role in advocating for a funding system, which decreases inconsistencies in the allocation of funding. 
Implications for theory. During the course of this study, it became apparent that there was lack of theoretical development on the concept of organizational capacity. More specifically, there was no theory that explored organizational capacity in relation to support for RU, from a healthcare, nursing perspective. Efforts in this area might also benefit from comparing and contrasting various conceptualizations of organizational capacity in order to develop a foundational understanding of the components which are important when exploring an organizations' capacity to support research use. Therefore, future efforts in this area should be focused on developing a theory which tests and clarifies the role of organizational capacity and its components when exploring supports for RU. Once developed, an organizational capacity theory may serve to guide future research efforts in this area, which is critical in order to gain a better understanding of how organizations' can support RU among nurses.

\section{Conclusion}

This descriptive, quantitative study was conducted to explore the presence of components of organizational capacity that support RU and also examine the facilitators and barriers to RU as reported by senior nursing leaders. Oxman and colleagues’ (2009) Organizational Capacity Instrument was used to collect information about seven components of organizational capacity throughout various organizations in Ontario. Study findings revealed that participants reported the presence of a culture and values that were supportive of research use and support for continuing professional development. However, the two components that participants reported as least frequently available in their organizations were setting priorities for obtaining research evidence and assessing quality and applicability of research and interpreting the results.

Furthermore, participants did not report support for adequate time, incentives, resources, expertise and arrangements with external experts. These results were echoed by responses to the 
open-ended questions, which revealed lack of resources, an unsupportive organization and nurse attitudes and beliefs as barriers to RU, whereas facilitators included networks, access to resources and organizational support. These findings have important implications for organizations and senior-nursing leaders as consistent mention of the barriers discussed above might indicate the presence of a systemic issue that has not been addressed. Until these issues are addressed, challenges with engaging nurses in RU will continue to persist.

The evidence produced from this study not only highlights the complexity of RU and the need for the presence of multiple components of organizational capacity to be supportive of RU, but it also highlights key priority areas for senior nursing leaders to explore further within their organizations. In order for organizational capacity to be supportive of research utilization, organizations need: an organizational culture that values RU, priorities for obtaining research, to obtain research, staff with the knowledge and skills to search, find and critically appraise evidence, to role-model the use of research, to monitor and evaluate continuously and provide professional development opportunities to nurses. The absence or presence of one component has the possibility of impacting other components. Senior-nursing leaders can potentially play a key role in addressing some of the issues discussed throughout this chapter and increase an organizations capacity to support RU at all levels within an organization. The study findings also raise implications for nursing practice, education, research, policy and theory. 


\section{Appendix A: First Literature Search Results}

\begin{tabular}{|c|c|c|c|}
\hline Database & Search terms & Limiters & \# of hits \\
\hline \multirow[t]{2}{*}{$\begin{array}{l}\text { Cumulative Index of } \\
\text { Nursing and Allied } \\
\text { Health (CINAHL) }\end{array}$} & $\begin{array}{l}\text { Organizational } \\
\text { capacity AND } \\
\text { (research utilization } \\
\text { OR research use) }\end{array}$ & $\begin{array}{l}\text { - English } \\
\text { - Published } 2004 \text { to } \\
2014\end{array}$ & 0 \\
\hline & $\begin{array}{l}\text { Organization* AND } \\
\text { capacity AND } \\
\text { (research utilization } \\
\text { OR research use) }\end{array}$ & $\begin{array}{l}\text { - English } \\
\text { - Published } 2004 \text { to } \\
2014\end{array}$ & 88 \\
\hline Ovid (Medline) & $\begin{array}{l}\text { Organizational } \\
\text { capacity AND } \\
\text { research utilization }\end{array}$ & $\begin{array}{l}\text { - English } \\
\text { - Published } 2004 \text { to } \\
2014\end{array}$ & 5 \\
\hline $\begin{array}{l}\text { ProQuest Nursing \& } \\
\text { Allied Health } \\
\text { Source }\end{array}$ & $\begin{array}{l}\text { Organizational } \\
\text { capacity AND } \\
\text { research utilization }\end{array}$ & $\begin{array}{l}\text { - English } \\
\text { - Peer-reviewed } \\
\text { - Scholarly journals } \\
\text { - Articles } \\
\text { - Published } 2004 \text { to } \\
2014\end{array}$ & 1136 \\
\hline $\begin{array}{l}\text { Business Source } \\
\text { Elite }\end{array}$ & $\begin{array}{l}\text { Organizational } \\
\text { capacity AND } \\
\text { definition }\end{array}$ & $\begin{array}{l}\text { - English } \\
\text { - Published } 2004 \text { to } \\
2014\end{array}$ & 355 \\
\hline $\begin{array}{l}\text { Canadian Business } \\
\text { and Current Affairs } \\
\text { (CBCA) Complete }\end{array}$ & $\begin{array}{l}\text { Organizational } \\
\text { capacity AND } \\
\text { definition }\end{array}$ & $\begin{array}{l}\text { - English } \\
\text { - Published } 2004 \text { to } \\
2014 \\
\text { - Peer reviewed } \\
\text { - Scholarly Journals }\end{array}$ & 3126 \\
\hline
\end{tabular}




\section{Appendix B: Second Literature Search Results}

\begin{tabular}{|c|c|c|c|}
\hline Database & Search terms & Limiters & \# of hits \\
\hline \multirow[t]{2}{*}{$\begin{array}{l}\text { Cumulative } \\
\text { Index of } \\
\text { Nursing and } \\
\text { Allied Health } \\
\text { (CINAHL) }\end{array}$} & $\begin{array}{l}\text { Nursing Practice, } \\
\text { Evidence-Based AND } \\
\text { Organizational } \\
\text { Culture }\end{array}$ & $\begin{array}{l}\text { - English } \\
\text { - Published } 2004 \text { to } 2014\end{array}$ & 178 \\
\hline & $\begin{array}{l}\text { (MH "Nursing } \\
\text { Practice, Research- } \\
\text { Based") OR (MH } \\
\text { "Nursing Practice, } \\
\text { Evidence-Based") } \\
\text { AND } \\
\text { (MH "Organizational } \\
\text { Culture") OR } \\
\text { ("context") }\end{array}$ & $\begin{array}{l}\text { - English Language } \\
\text { - Peer Reviewed } \\
\text { - Published } 2004 \text { to } 2014 \\
\text { - Special Interest: } \\
\text { Evidence-Based Practice } \\
\text { - Any Author is Nurse } \\
\text { (Reduced to n=55) }\end{array}$ & 318 \\
\hline \multirow[t]{3}{*}{$\begin{array}{l}\text { Ovid } \\
\text { (Medline) }\end{array}$} & $\begin{array}{l}\text { Research utilization } \\
\text { AND (organizational } \\
\text { context OR } \\
\text { organizational context) }\end{array}$ & - Published 2004 to 2014 & 54 \\
\hline & $\begin{array}{l}\text { Evidence-based } \\
\text { practice AND context } \\
\text { AND nurs* }\end{array}$ & $\begin{array}{l}\text { - English language } \\
\text { - Published } 2004 \text { to } 2014\end{array}$ & 101 \\
\hline & $\begin{array}{l}\text { Research utilization } \\
\text { AND }(\text { organization } \\
\text { OR context })\end{array}$ & - None & 4 \\
\hline $\begin{array}{l}\text { ProQuest } \\
\text { Nursing \& } \\
\text { Allied Health } \\
\text { Source }\end{array}$ & $\begin{array}{l}\text { Research utilization } \\
\text { AND organizational } \\
\text { context AND nursing }\end{array}$ & $\begin{array}{l}\text { - Scholarly Journals } \\
\text { - Published } 2004 \text { to } 2014\end{array}$ & 885 \\
\hline $\begin{array}{l}\text { EBM Reviews } \\
\text { - Cochrane } \\
\text { Methodology } \\
\text { Register }\end{array}$ & $\begin{array}{l}\text { (Evidence-based } \\
\text { nursing OR evidence- } \\
\text { based medicine) OR } \\
\text { research utilization } \\
\text { AND nurs* }\end{array}$ & - Published 2004 to 2014 & 38 \\
\hline
\end{tabular}




\section{Appendix C: Online NLN.ON Recruitment Advertisement}

\section{Nursing Leaders' Perspectives: An Exploration of Organizational Structures and Processes that Impact Research Utilization among Nurses}

Are you a nursing leader (Chief Nursing Officer, Director of Nursing, Assistant Director of Nursing, Manager, President, Vice President) working in Ontario?

If so, you may be eligible to participate in this research study.

The purpose of this study is to:

Examine the organizational structures and processes that influence nurses' engagement in research utilization across various healthcare organizations in Ontario and explore the facilitators and barriers of research utilization from the perspective of the nursing leader.

What will you be asked to do?

Your participation will include completing an online questionnaire asking your perspective on organizational factors influencing research utilization. The questionnaire is anonymous and will take approximately $\mathbf{2 0}$ minutes of your time.

Who is completing the study?

Tanvi Sharma is conducting the study as part of the thesis requirements for the Master of Nursing Degree at Ryerson University.

Need more information? How can you participate?

Please contact the researcher by email at tanvi.sharma@ ryerson.ca

If you are interested in participating in the study please visit

https://survey.ryerson.ca:443/s?s=3311

Please note: Detailed information and the consent process will be outlined online, prior to beginning the questionnaires, should you choose to participate.

I thank you for your consideration of this invitation to participate in a research study.

Approved by Ryerson Research Ethics Board: March 2014 


\section{Appendix D: Ryerson University Research Ethics Board Approval}

\section{RYERSONUNIVERSITY}

\section{RESEARCH ETHICS BOARD}

To: Tanvi Sharma

Nursing (DCSN)

Re: REB 2014-079: Nursing Leaders' Perspectives: An Exploration of Organizational Structures and Processes that Impact Research Utilization Among Nurses

Date: March 17, 2014

\section{Dear Tanvi Sharma,}

The review of your protocol REB File REB 2014-079 is now complete. The project has been approved for a one year period. Please note that before proceeding with your project, compliance with other required University approvals/certifications, institutional requirements, or governmental authorizations may be required.

This approval may be extended after one year upon request. Please be advised that if the project is not renewed, approval will expire and no more research involving humans may take place. If this is a funded project, access to research funds may also be affected.

Please note that REB approval policies require that you adhere strictly to the protocol as last reviewed by the REB and that any modifications must be approved by the Board before they can be implemented. Adverse or unexpected events must be reported to the REB as soon as possible with an indication from the Principal Investigator as to how, in the view of the Principal Investigator, these events affect the continuation of the protocol.

Finally, if research subjects are in the care of a health facility, at a school, or other institution or community organization, it is the responsibility of the Principal Investigator to ensure that the ethical guidelines and approvals of those facilities or institutions are obtained and filed with the REB prior to the initiation of any research.

Please quote your REB file number (REB 2014-079) on future correspondence.

Congratulations and best of luck in conducting your research.

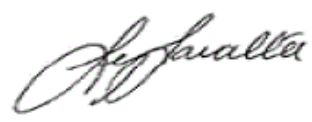

Lynn Lavallée, Ph.D.

Chair, Research Ethics Board 


\section{Appendix E: Letter of Information}

\section{Daphne Cockwell SCHOOL OF NURSING}

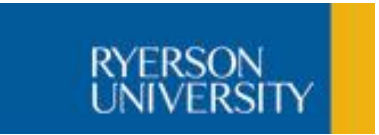

\section{Nursing Leaders' Perspectives: An Exploration of Organizational Structures and Processes that Impact Research Utilization Among Nurses}

You are being invited to participate in a research study. Please read this Consent Form so that you understand what your participation will involve. Before you consent to participate, please ask any questions necessary to be sure you understand what your participation will involve.

Investigators: This research is being conducted by Tanvi Sharma, RN, BScN, MN (student) from the Daphne Cockwell School of Nursing as part of her thesis requirements for the Master of Nursing Degree at Ryerson University. This study is being conducted under the supervision of Dr. Cristina Catallo, Associate Professor, Daphne Cockwell School of Nursing, Ryerson University.

Purpose of the Study: You are invited to participate in a research study which seeks to examine the organizational structures and processes that influence nurses' engagement in research utilization across various healthcare organizations in Ontario. Specifically, the facilitators and barriers to research utilization will be examined from the nurse leaders' perspective.

Description of the Study: If you volunteer to participate in this study, you will be asked to complete a demographic data questionnaire which includes background questions about you such as sex, age, and your nursing experience. You will also be asked to complete The Organizational Capacity to Support the Use of Research Evidence to Inform Decisions Instrument which explores organizational structures and processes, assesses how they influence research utilization among nurses. And at the end, answer two open-ended questions, which aim to explore the facilitators and barriers to research use. It is anticipated that time required to complete the questionnaires is approximately 20 minutes. The questionnaires can be completed at your convenience, in any setting and at any time that you prefer.

Risks or Discomforts: The research team anticipates that there is a low level of harm and potential risk from participating in this research study. Once you have started the questionnaire, should you feel uncomfortable answering any questions, at any time, please feel free to skip any question(s) (by leaving them blank) or stop the study (by closing the browser). In the situation that you wish to quit the study once consent has been given, only the responses you have completed up to that point will be received by the researcher. And only these received responses will be included in the study. Completion of both these questionnaires is completely anonymous, as you will not be asked for your name anywhere. There will be no way to link your responses to you or the organization in which you are employed. Therefore, please do not include any information that may identify you or the organization for which you work on any part of the questionnaires. Please note because this study is anonymous, you cannot withdraw your 
responses once you have completed the questionnaires. As mentioned previously, if you decide to quit the study at any point, only your responses made up to that point will visible to the researcher and included in the study.

Benefits of the Study: Although there are benefits to participants, the researcher anticipates findings from this study will contribute to knowledge generation about research utilization. This knowledge may help organizations assess how they support or hinder the nurses' use of research and potentially develop strategies to support use of research in practice.

Confidentiality: Responses from questionnaires will be kept on a password-protected computer with password-protected files for one year then deleted off the computer permanently. Access to responses will be limited to only those on the research team. The findings of this study will be shared at various conferences, in professional health journals and utilized for fulfilling thesis requirement purposes, however only grouped responses will be reported. Participant responses are anonymous and reported only in aggregate to ensure confidentiality.

Incentives to Participate: There are no monetary incentives to participate.

Voluntary Nature of Participation: The decision to participate in this study is completely voluntary and you have the right to refuse participation. Should you choose not to participate, please note this will not affect your future relationship or interactions with Ryerson University, the Nursing Leadership Network of Ontario, your employer or anyone involved in the research study. Should you choose to participate, you also have the option to withdraw from the study at any time without penalty or loss.

Questions about the Study: If you require additional information or have comments, questions or concerns (at any time) please feel free to contact Tanvi Sharma at tanvi.sharma@ryerson.ca or Dr. Cristina Catallo, Associate Professor, Daphne Cockwell School of Nursing, Ryerson University at ccatallo@ryerson.ca or 416-979-5000 extension 2019.

This study has been approved by Ryerson University's Research Ethics Board. If you have questions regarding your rights as a research participant, please contact:

Toni Fletcher, Research Ethics Coordinator

1 Dundas Street West, 11th Floor

Toronto, Ontario M5G $1 \mathrm{Z3}$

(416)979-5000 ext. 7112

toni.fletcher@ryerson.ca

Please note: Consent to participate in this study is implied by answering the question below and submitting the questionnaires online.

Please note: This study is $\underline{\mathbf{O N L Y}}$ seeking participants in senior nursing leadership positions (Chief Nursing Officer, Director of Nursing, Assistant Director of Nursing, Manager, President, Vice President). If you are not employed in a senior nursing leadership position, please do not continue the survey. 
In addition, please note that if you agree to participate (by answering yes to the question below), your given responses will be used regardless of if you choose to:

- Complete all survey questions and click submit at the end

- Skip one or more survey question[s] but proceed to the end of the survey and click submit

- Stop the survey at any point (by closing the browser), and do not click submit at the end

Answering yes to the question below will indicate that you have read and agree with the information on this page. If you answer no to the question below, you will be exited from the system.

Do you consent to participate in this survey?
○ YES

O NO 


\section{Appendix F: Demographic Data Questionnaire}

\section{Nursing Leaders' Perspectives: An Exploration of Organizational Structures and Processes}

that Impact Research Utilization Among Nurses

\section{DEMOGRAPHIC DATA QUESTIONNAIRE}

Dear Nursing Leader,

If you currently work as a front-line staff/charge/resource/advanced practice nurse, team leader, educator, nurse practitioner or are in a temporary leadership position, we thank you for your time, but you do not meet the study eligibility criteria. Please do not continue with the study.

1. Please indicate your sex

○ Male

○ Female

2. How old are you

3. How many years have you been a registered nurse?

4. Please indicate the highest level of nursing education you have completed

- Diploma in Nursing

- Baccalaureate in Nursing

- Masters in Nursing

$\circ \quad$ PhD in Nursing

O Other, please specify:

5. Please indicate the highest level of non-nursing education you have completed

○ Diploma

- Baccalaureate

- Masters

○ $\mathrm{PhD}$

- Not applicable

○ Other, please specify:

6. How many years have you been in a leadership position?

7. What is your current position?

$\circ \quad$ Chief Nursing Officer

- Director of Nursing/Assistant Director of Nursing

- Manager

- President/ Vice President

○ Other, please specify:

8. How many years have you practiced in your current position? 
9. In which type of setting do you currently practice?

- Acute care hospital

- Community organization

- Long-term care facility

O Other, please specify:

10. How would you classify the size of your organization?

$\circ \quad<5$ nursing staff

$\circ \quad 5-10$ nursing staff

○ $11-20$ nursing staff

○ 21-30 nursing staff

○ $\quad 31-40$ nursing staff

○ 41-50 nursing staff

$\circ \quad>50$ nursing staff 


\section{References}

Aitken, L., Hackwood, B., Crouch, S., Clayton, S., West, N., Carney, D., \& Jack, L. (2011). Creating an environment to implement and sustain evidence based practice: A developmental process. Australian Critical Care, 24(4), 244-254. doi: 10.1016/j.aucc.2011.01.004

American Nurses Credentialing Center (ANCC). (2013). ANCC Magnet Recognition Program. Retrieved from http://www.nursecredentialing.org/Magnet

Barnsteiner, J. H., Reeder, V. C., Palma, W. H., Preston, A. M., \& Walton, M. K. (2010). Promoting evidence-based practice and translational research. Nursing Administration Quarterly, 34(3), 217. doi: 10.1097/NAQ.0b013e3181e702f4.

Bartelt, T. C., Ziebert, C., Sawin, K. J., Malin, S., Nugent, M., \& Simpson, P. (2011). Evidencebased practice: Perceptions, skills, and activities of pediatric health care professionals. Journal of Pediatric Nursing, 26(2), 114-121. doi:10.1016/j.pedn.2010.12.003

Billingham, S. A. M., Whitehead, A. L., \& Julious, S. A. (2013). An audit of sample sizes for pilot and feasibility trials being undertaken in the United Kingdom registered in the United Kingdom Clinical Research Network database. BMC Medical Research Methodology, 13(104), 1-6. doi:10.1186/1471-2288-13-104

Bonner, A., \& Sando, J. (2008). Examining the knowledge, attitude and use of research by nurses. Journal of Nursing Management, 16(3), 334-343. doi:10.1111/j.13652834.2007.00808.x 
Boswell, C., \& Cannon, S. (2007). Introduction to nursing research: Incorporating evidencebased practice. Burlington, MA: Jones \& Bartlett Learning

Bowen, S., Erickson, T., Martens, P. J., \& Crockett, S. (2009). More than "Using Research": The real challenges in promoting Evidence-Informed Decision-Making. Healthcare Policy, 4(3), 87-102.

Breimaier, H. E., Halfens, R. J. G., \& Lohrmann, C. (2011). Nurses' wishes, knowledge, attitudes and perceived barriers on implementing research findings into practice among graduate nurses in Austria. Journal of Clinical Nursing, 20(11-12), 1744-1756. doi:10.1111/j.1365-2702.2010.03491.x

Brown, C. E., Wickline, M. A., Ecoff, L., \& Glaser, D. (2009). Nursing practice, knowledge, attitudes and perceived barriers to evidence-based practice at an academic medical center. Journal of Advanced Nursing, 65(2), 371-381. doi:10.1111/j.1365-2648.2008.04878.x

Browne, R. H. (1995). On the use of a pilot sample for sample size determination. Statistics in Medicine, 14(17), 1933-1940. doi:10.1002/sim.4780141709

Burns, H.K., Dudjak, L., \& Greenhouse, P.K. (2009). Building an evidence-based practice infrastructure and culture: A model for rural and community hospitals. The Journal of Nursing Administration, 39(7/8), 321-325. doi: 10.1097/NNA.0b013e3181ae966a

Burns, N., \& Grove, S. K. (2009). The practice of nursing research: Appraisal, synthesis, and generation of evidence. St. Louis, Mo: Saunders/Elsevier. 
Cadmus, E., Van Wynen, E. A., Chamberlain, B., Steingall, P., Kilgallen, M. E., Holly, C., \& Gallagher-Ford, L. (2008). Nurses' skill level and access to evidence-based practice. The Journal of Nursing Administration, 38(11), 494-503.

doi:10.1097/01.NNA.0000339471.42596.18

Canadian Nurses Association. (2010). Position statement: Evidence-informed decision-making and nursing practice. Retrieved from http://www.nanb.nb.ca/PDF/CNAEvidence_Informed_Decision_Making_and_Nursing_Practice_E.pdf

Catallo, C., \& Sidani, S. (2014). The self-assessment for organizational capacity instrument for evidence-informed health policy: Preliminary reliability and validity of an instrument. Worldviews on Evidence-Based Nursing, 11(1), 35-45. doi:10.1111/wvn.12018

Chau, J. P. C., Lopez, V., \& Thompson, D. R. (2008). A survey of Hong Kong nurses' perceptions of barriers to and facilitators of research utilization. Research in Nursing \& Health, 31(6), 640-649. doi:10.1002/nur.20289

Chummun, H. \& Tiran, D. (2008). Increasing research evidence in practice: a possible role for the consultant nurse. Journal of Nursing Management, 16(3), 327-333. doi: 10.1111/j.1365-2834.2007.00791.x

Ciliska, D. (2012). Introduction to Evidence-Informed Decision Making. Retrieved from http://www.cihr-irsc.gc.ca/e/45245.html 
Cohen, B. E., Schultz, A., McGibbon, E., VanderPlaat, M., Bassett, R., GermAnn, K., ... Fuga, L. A. (2013). A conceptual framework of organizational capacity for public health equity action. Canadian Journal of Public Health, 104(3), e262-e266.

College of Nurses of Ontario. (2013). Data Query Tool. Retrieved from http://www.cno.org/what-is-cno/nursing-demographics/data-query-tool/

College of Nurses of Ontario. (2014a). Competencies for entry-level registered nurse practice. Retrieved from http://www.cno.org/Global/docs/reg/41037_EntryToPracitic_final.pdf?epslanguage=en

College of Nurses of Ontario. (2014b). Membership Statistics Highlights 2014. Retrieved from http://www.cno.org/Global/docs/general/43069_stats/43069_MembershipStatisticsHighlights.pdf

College of Nurses of Ontario. (2002). Professional Standards. Retrieved from http://www.cno.org/Global/docs/prac/41006_ProfStds.pdf

Corchon, S. (2010). Commentary on Kocaman G, Seren S, Lash AA, Kurt S, Bengu N \&Yurumezoglu HA. Barriers to research usage by staff nurses in a university hospital. Journal of Clinical Nursing 19, 1908-1918. Journal of Clinical Nursing, 19(13-14), 2076-2078. doi:10.1111/j.1365-2702.2010.03207.x

Creswell, J.W. (2007). Qualitative inquiry and research design: Choosing among five approaches (2nd ed.). Thousand Oaks, CA: Sage Publications Inc. 
Cullum, N., Ciliska, D., Haynes, B. R., \& Marks, S. (2008). Evidence-based nursing: An introduction (1st ed.). Oxford, UK: Blackwell.

Cummings, G. G., Estabrooks, C. A., Midodzi, W. K., Wallin, L., \& Hayduk, L. (2007). Influence of organizational characteristics and context on research utilization. Nursing Research, 56(4 Suppl), S24-S39. doi:10.1097/01.NNR.0000280629.63654.95

Dillman, D. A., Smyth, J. D., \& Christian, L. M. (2009). Internet, mail, and mixed-mode surveys: The tailored design method. Hoboken, NJ: Wiley \& Sons.

Drenkard, K. (2013). Creating a culture for advancing nursing research. The Journal of Nursing Administration, 43(5), 245-246. doi:10.1097/NNA.0b013e31828eeb86

Ellen, M. E., Léon, G., Bouchard, G., Lavis, J. N., Ouimet, M., \& Grimshaw, J. M. (2013). What supports do health system organizations have in place to facilitate evidence-informed decision-making? A qualitative study. Implementation Science, 8(84), 1-19. doi:10.1186/1748-5908-8-84

Ellen, M. E., Léon, G., Bouchard, G., Ouimet, M., Grimshaw, J. M. \& Lavis, J. N. (2014). Barriers, facilitators and views about next steps to implementing supports for evidenceinformed decision-making in health systems a qualitative study. Implementation Science, 9(1), 1-12. doi:10.1186/s13012-014-0179-8

Elo, S., \& Kyngäs, H. (2008). The qualitative content analysis process. Journal of Advanced Nursing, 62(1), 107-115. doi:10.1111/j.1365-2648.2007.04569.x 
Estabrooks C.A. (2003). Translating research into practice: Implications for organizations and administrators. Canadian Journal of Nursing Research, 35(3), 53-68.

Estabrooks, C. A. (1998). Will evidence-based nursing practice make practice perfect? The Canadian Journal of Nursing Research, 30(1), 15-36.

Estabrooks, C. A., Floyd, J. A., Scott-Findlay, S., O'Leary, K. A., \& Gushta, M. (2003). Individual determinants of research utilization: A systematic review. Journal of Advanced Nursing, 43(5), 506-520. doi:10.1046/j.1365-2648.2003.02748.x

Estrada, N. (2009). Exploring perceptions of a learning organization by RNs and relationship to EBP beliefs and implementation in the acute care setting. Worldviews on Evidence-Based Nursing, 6(4), 200- 209. doi:10.1111/j.1741-6787.2009.00161.x

Fox-Wasylyshyn, S. M., \& El-Masri, M. M. (2005). Handling missing data in self-report measures. Research in Nursing \& Health, 28(6), 488-495. doi:10.1002/nur.20100

Gerrish, K., Ashworth, P., Lacey, A., \& Bailey, J. (2008). Developing evidence-based practice: Experiences of senior and junior clinical nurses. Journal of Advanced Nursing, 62(1), 6273. doi:10.1111/j.1365-2648.2007.04579.x

Gerrish, K., Nolan, M., McDonnell, A., Tod, A., Kirshbaum, M., \& Guillaume, L. (2012). Factors influencing advanced practice nurses' ability to promote evidence-based practice among frontline nurses. Worldviews on Evidence-Based Nursing, 9(1), 30-39. doi:10.1111/j.1741-6787.2011.00230.x 
Hart, P., Eaton, L., Buckner, M., Morrow, B., Barrett, D., Fraser, D., \& ... Sharrer, R. (2008). Effectiveness of a computer-based educational program on nurses' knowledge, attitude, and skill level related to evidence-based practice. Worldviews On Evidence-Based Nursing, 5(2), 75-84. doi: 10.1111/j.1741-6787.2008.00123.x.

Hamel, N., \& Schrecker, T. (2011). Unpacking capacity to utilize research: A tale of the Burkina Faso public health association. Social Science \& Medicine, 72(1), 31-38. doi:10.1016/j.socscimed.2010.09.051

Hauck, S., Winsett, R. P., \& Kuric, J. (2013). Leadership facilitation strategies to establish evidence-based practice in an acute care hospital. Journal of Advanced Nursing, 69(3), 664-674. doi:10.1111/j.1365-2648.2012.06053.x

Hsieh, H., \& Shannon, S. E. (2005). Three approaches to qualitative content analysis. Qualitative Health Research, 15(9), 1277-1288. doi:10.1177/1049732305276687

Hutchinson, A. M., \& Johnston, L. (2006). Beyond the BARRIERS scale: Commonly reported barriers to research use. The Journal of Nursing Administration, 36(4), 189-199. doi:10.1097/00005110-200604000-00008

International Council of Nurses. (2012). Closing the gap: From evidence to action. Retrieved from http://www.nursingworld.org/MainMenuCategories/ThePracticeofProfessionalNursing/I mproving-Your-Practice/Research-Toolkit/ICN-Evidence-Based-PracticeResource/Closing-the-Gap-from-Evidence-to-Action.pdf 
Jeffs, L., Smith, O., Beswick, S., Maoine, M., \& Ferris, E. (2013). Investing in nursing research in practice settings: A blueprint for building capacity. Canadian Journal Of Nursing Leadership, 26(4), 44-59.

Jolley, J. (2013). Introducing Research and Evidence-Based Practice for Nursing \& Healthcare Professionals. Abingdon, Oxon: Routledge.

Julious, S. A. (2005). Sample size of 12 per group rule of thumb for a pilot study. Pharmaceutical Statistics, 4(4), 287-291. doi:10.1002/pst.185

Karkos, B., \& Peters, K. (2006). A magnet community hospital: Fewer barriers to nursing research utilization. The Journal of Nursing Administration, 36(78), 377-382.

Ketefian, S. (1975). Application of selected nursing research findings into nursing practice: a pilot study. Nursing Research, 24(2), 89-92.

Kitson, A., Silverston, H., Wiechula, R., Zeitz, K., Marcoionni, D., \& Page, T. (2011). Clinical nursing leaders', team members' and service managers' experiences of implementing evidence at a local level. Journal of Nursing Management, 19(4), 542-555. doi:10.1111/j.1365-2834.2011.01258.x

Kocaman, G., \& Lash, A. A. (2011). Research utilisation, organisational research culture and Corchon hypothesis: Response to Corchon S (2010) commentary on Kocaman G, Seren S, Lash AA, Kurt S, Bengu N \& Yurumezoglu HA (2010) barriers to research usage by staff nurses in a university hospital. Journal of clinical nursing 19, 2076-2078. Journal of Clinical Nursing, 20(13-14), 2092-2093. doi:10.1111/j.1365-2702.2010.03603.x 
Kocaman, G., Seren, S., Lash, A. A., Kurt, S., Bengu, N., \& Yurumezoglu, H. A. (2010). Barriers to research utilisation by staff nurses in a university hospital: Barriers to research utilisation. Journal of Clinical Nursing, 19(13-14), 1908-1918. doi:10.1111/j.13652702.2009.03032.x

Koehn, M. L., \& Lehman, K. (2008). Nurses' perceptions of evidence-based nursing practice. Journal of Advanced Nursing, 62(2), 209-215. doi:10.1111/j.1365-2648.2007.04589.x

Krugman, M. (2010). Evidence-based practice and the magnet journey. Journal for Nurses in Staff Development, 26(5), 239-241.

Lavis, J.N. (2006). Research, public policymaking, and knowledge translation processes: Canadian efforts to build bridges. The Journal of Continuing Education in the Health Professions, 26(1), 37-45.

Lavis, J.N., \& Catallo, C. (Eds.). (2013). Bridging the Worlds of Research and Policy in European Health Systems. Copenhagen, Denmark: World Health Organization. Retrieved from http://www.euro.who.int/en/aboutus/partners/observatory/publications/studies/bridging-the-worlds-of-research-and-policyin-european-health-systems

Lavis, J.N., Davies, H., Denis, J.L., Golden-Biddle, \& Ferlie, E. (2005). Towards systematic reviews that inform health care management and policy-making. Journal of Health Services Research and Policy, 10(Suppl 1), 35-48. 
Lavis, J. N., Lomas, J., Hamid, M., \& Sewankambo, N. K. (2006). Assessing country-level efforts to link research to action. Bulletin of the World Health Organization, 84(8), 620628. doi:10.2471/BLT.06.030312

Lavis, J. N., Oxman, A. D., Lewin, S., \& Fretheim, A. (2009). SUPPORT tools for evidenceinformed health policymaking (STP) 1. Health Research Policy and Systems, 7(Suppl 1), I1-I1. doi: 10.1186/1478-4505-7-S1-I1

Liang, Z., Howard, P.F., Leggat, S.G., \& Murphy, G. (2012). A framework to improve evidenceinformed decision-making in health service management. Australian Health Review, 36(3), 284-289. doi: 10.1071/AH11051

Loiselle, C. G., Profetto-McGrath, J., Polit, D. F., \& Beck, C. T. (2007). Canadian essentials of nursing research (2nd ed.). Philadelphia, PA: Lippincott Williams \& Wilkins.

Loke, J. C. F., Laurenson, M. C., \& Lee, K. W. (2014). Embracing a culture in conducting research requires more than nurses' enthusiasm. Nurse Education Today, 34(1), 132-137. doi:10.1016/j.nedt.2012.09.006

Martella, R.C., Ron Nelson, J., Morgan, R.L., \& Marchand-Martella, N.E. (2013). Understanding and Interpreting Educational Research. New York, NY: The Guilford Press.

Matthew-Maich, N., Ploeg, J., Jack, S., \& Dobbins, M. (2010). Transformative learning and research utilization in nursing practice: A missing link? Worldviews on Evidence-Based Nursing, 7(1), 25-35. doi:10.1111/j.1741-6787.2009.00172.x 
McCormack, B., Rycroft-Malone, J., DeCorby, K., Hutchinson, A. M., Bucknall, T., Kent, B., ... Wilson, V. (2013). A realist review of interventions and strategies to promote evidenceinformed healthcare: A focus on change agency. Implementation Science, 8, 107. doi:10.1186/1748-5908-8-107

McEwen, M., \& Wills, E. M. (2011). Theoretical basis for nursing (3rd ed.). Philadelphia: Lippincott Williams \& Wilkins.

McMaster University. (2015). McMaster Evidence-Based Clinical Practice Workshops. Retrieved from http://ebm.mcmaster.ca/index.htm

Melnyk, B. M., Fineout-Overholt, E., Gallagher-Ford, L., \& Kaplan, L. (2012). The state of evidence-based practice in US nurses: Critical implications for nurse leaders and educators. The Journal of Nursing Administration, 42(9), 410-417. doi:10.1097/NNA.0b013e3182664e0a

Melnyk, B. M., Fineout-Overholt, E., Giggleman, M., \& Cruz, R. (2010). Correlates among cognitive beliefs, EBP implementation, organizational culture, cohesion and job satisfaction in evidence-based practice mentors from a community hospital system. Nursing Outlook, 58(6), 301-308. doi:10.1016/j.outlook.2010.06.002

Mensik, J. S. (2011). Understanding research and evidence-based practice: From knowledge generation to translation. Journal of Infusion Nursing, 34(3), 174-178. doi:10.1097/NAN.0b013e3182134f44 
Meyer, A., Davis, M., \& Mays, G. P. (2012). Defining organizational capacity for public health services and systems research. Journal of Public Health Management and Practice, $18(6), 535-544$.

Missal, B., Schafer, B. K., Halm, M. A., \& Schaffer, M. A. (2010). A university and health care organization partnership to prepare nurses for evidence-based practice. The Journal of Nursing Education, 49(8), 456-461. doi:10.3928/01484834-20100430-06

Myers, G., \& Meccariello, M. (2006). From pet rock to rock-solid: Implementing unit-based research. Nursing Management, 37(1), 24-29. doi:10.1097/00006247-200601000-00007

National Collaborating Centre for Methods and Tools (NCCMT). (2012). NCCMT Learning Centre: What is Evidence-Informed Public Health? Retrieved from http://www.nccmt.ca/learningcentre/index.php?lang=en\#main3.html

Ontario Ministry of Finance. (2014). Public Sector Salary Disclosure 2014 (Disclosure for 2013). Retrieved from http://www.fin.gov.on.ca/en/publications/salarydisclosure/pssd/pdf/hospitals_2013.pdf

Oxman, A. D., Vandvik, P. O., Lavis, J. N., Fretheim, A., \& Lewin, S. (2009). SUPPORT tools for evidence-informed health policymaking (STP) 2: Improving how your organisation supports the use of research evidence to inform policymaking. Health Research Policy and Systems, 7(Suppl 1), S2. doi:10.1186/1478-4505-7-S1-S2

Parkosewich, J. A. (2013). An infrastructure to advance the scholarly work of staff nurses. The Yale Journal of Biology and Medicine, 86(1), 63. 
Peirson, L., Ciliska, D., Dobbins, M., \& Mowat, D. (2012). Building capacity for evidence informed decision making in public health: A case study of organizational change. Implementation Science, 12(2), 1-13. doi: 10.1186/1471-2458-12-137

Penz, K.L., \& Bassendowski, S.L. (2006). Evidence-based nursing in clinical practice: Implications for nurse educators. The Journal of Continuing Education in Nursing, 37(6), 250-254.

Polit, F. D. (2010). Statistics and Data Analysis for Nursing Research (2 ${ }^{\text {nd }} e d$.). Bergen, NJ: Pearson Education.

Pravikoff, D. S., Tanner, A. B., \& Pierce, S. T. (2005). Readiness of U.S. nurses for evidencebased practice. The American Journal of Nursing, 105(9), 40-51.

Roe, E. \& Whyte-Marshall, M. (2012). Mentoring for evidence-based practice: A collaborative approach. Journal for Nurses in Staff Development, 28(4), 177-181. doi: 10.1097/NND.0b013e31825dfb2a

Roxburgh, M. (2006). An exploration of factors which constrain nurses from research participation. Journal of Clinical Nursing, 15(5), 535-545. doi:10.1111/j.13652702.2006.01374.x

Rutledge, D. N., \& Donaldson, N. E. (1995). Building organizational mechanisms for research utilization. Journal of Nursing Administration, 25(10), 12-16.

Rycroft-Malone, J. (2008). Evidence-informed practice: From individual to context. Journal of Nursing Management, 16(4), 404-408. doi:10.1111/j.1365-2834.2008.00859.x 
Rycroft-Malone, J., Harvey, G., Seers, K., Kitson, A., McCormack, B., \& Titchen, A. (2004). An exploration of the factors that influence the implementation of evidence into practice. Journal of Clinical Nursing, 13(8), 913-924. doi:10.1111/j.1365-2702.2004.01007.x

Salmela, S., Eriksson, K., \& Fagerström, L. (2013). Nurse leaders' perceptions of an approaching organizational change. Qualitative Health Research, 23(5), 689-699. doi: $10.1177 / 1049732313481501$

Sandström, B., Borglin, G., Nilsson, R., \& Willman, A. (2011). Promoting the implementation of evidence-based practice: A literature review focusing on the role of nursing leadership. Worldviews on Evidence-Based Nursing, 8(4), 212-223. doi:10.1111/j.17416787.2011.00216.x

Schein, E. (1992). Organizational Culture and Leadership (2nd ed.). San Francisco, CA: JosseyBass.

Scott, S. D., \& Pollock, C. (2008). The role of nursing unit culture in shaping research utilization behaviors. Research in Nursing \& Health, 31(4), 298-309. doi:10.1002/nur.20264

Scott-Findlay, S., \& Estabrooks, C. A. (2006). Mapping the organizational culture research in nursing: A literature review. Journal of Advanced Nursing, 56(5), 498-513. doi:10.1111/j.1365-2648.2006.04044.x

Scott-Findlay, S., \& Golden-Biddle, K. (2005). Understanding how organizational culture shapes research use. The Journal of Nursing Administration, 35(7-8), 359-365. doi:10.1097/00005110-200507000-00008 
Shea, C. M., Malone, R., Weinberger, M., Reiter, K. L., Thornhill, J., Lord, J., . . Weiner, B. J. (2014). Assessing organizational capacity for achieving meaningful use of electronic health records. Health Care Management Review, 39(2), 124-133. doi:10.1097/HMR.0b013e3182860937

Sim, J., \& Lewis, M. (2012). The size of a pilot study for a clinical trial should be calculated in relation to considerations of precision and efficiency. Journal of Clinical Epidemiology, 65(3), 301- 308. doi:10.1016/j.jclinepi.2011.07.011

Smith, J. R., \& Donze, A. (2010). Assessing environmental readiness: First steps in developing an evidence-based practice implementation culture. The Journal of Perinatal \& Neonatal Nursing, 24(1), 61-71. doi:10.1097/JPN.0b013e3181ce1357

Speziale, H., \& Carpenter, D. (2007). Qualitative research in nursing: Advancing the humanistic imperative (4th ed.). Philadelphia, PA: Lippincott Williams \& Wilkins.

Squires, J.E., Estabrooks, C.A., Gustavsson, P., \& Wallin, L. (2011). Individual determinants of research utilization by nurses: A systematic review update. Implementation Science, 6(1), 1-20. doi: 10.1186/1748-5908-6-1

Squires, J. E., Reay, T., Moralejo, D., Lefort, S. M., Hutchinson, A. M., \& Estabrooks, C. A. (2012). Designing strategies to implement research-based policies and procedures: A set of recommendations for nurse leaders based on the PARiHS framework. The Journal of Nursing Administration, 42(5), 293-297. doi:10.1097/NNA.0b013e318253565f 
Stallard, N. (2012). Optimal sample sizes for phase II clinical trials and pilot studies. Statistics in Medicine, 31(11-12), 1031-1042. doi: 10.1002/sim.4357

Straus, S. E., Tetroe, J., \& Graham, I. D. (2009). Knowledge translation in health care: Moving from evidence to practice. Chichester, UK: Wiley-Blackwell/BMJ.

Tavakol, M., \& Dennick, R. (2011). Making sense of Cronbach's alpha. International Journal of Medical Education, 2(1), 53-55. doi:10.5116/ijme.4dfb.8dfd

Tetroe, J. (2007). Knowledge translation at the Canadian institutes of health research: A primer. National Center for the Dissemination of Disability Research, 18(1), 1-8.

Thiel, L., \& Ghosh, Y. (2008). Determining registered nurses' readiness for evidence-based practice. Worldviews on Evidence-Based Nursing, 5(4), 182-192. doi:10.1111/j.17416787.2008.00137.x

Thompson, D. (2003). Fostering a research culture in nursing. Nursing Inquiry, 10(3), 143-144. doi: 10.1046/j.1440-1800.2003.00175.x

Tod, A., Palfreyman, S., \& Burke, L. (2004). Evidence-based practice is a time of opportunity for nursing. British Journal of Nursing, 13(4), 211-216.

Wallis, L. (2012). Barriers to implementing evidence-based practice remain high for U.S. nurses. American Journal of Nursing, 112(12), 15. doi: 10.1097/01.NAJ.0000423491.98489.70 
Wilkinson, J. E., Nutley, S. M., \& Davies, H. T. O. (2011). An exploration of the roles of nurse managers in evidence-based practice implementation. Worldviews on Evidence-Based Nursing, 8(4), 236-246. doi:10.1111/j.1741-6787.2011.00225.x

Yoder, L. H. (2005). Evidence-based practice: The time is now. Medsurg Nursing, 14(2), 91-92.

Yost, J., Ciliska, D., \& Dobbins, M. (2014). Evaluating the impact of an intensive education workshop on evidence-informed decision making knowledge, skills, and behaviours: A mixed methods study. BMC Medical Education, 14(1), 1-9. doi: 10.1186/1472-6920-1413 